\begin{tabular}{|c|c|c|}
\hline Beitr. Ent. & Keltern & ISSN 0005-805X \\
\hline $\mathbf{5 2}(2002) 1$ & S. $111-204$ & 31.08 .2002 \\
\hline
\end{tabular}

\title{
A taxonomic and phylogenetic revision of Amarochara THOMSON. I. The species of the Holarctic region
}

\section{(Coleoptera: Staphylinidae, Aleocharinae, Oxypodini)}

With 122 figure drawings, 4 diagrams, 8 maps, and 1 table

\section{VOLKER ASSING}

\section{Summary}

The types and additional material of the Amarochara species occurring in the Holarctic region are revised. 25 valid species are recognized, 24 of them (re-)described: $A$. umbrosa (ERICHSON), A. beterogaster CAMERON, A. sororcula CAMERON, A. inquilina (CASEY), A. fenyesi BLATCHLEY, A. brevios sp. n., $A$. bonnairei (FAUVEE), A. cribripennis (MULSANT \& REY), A. siculifera sp. n., A. inermis sp. n., A. caeca sp. n., A. crassicornis (QUEDENFELDT), A. forticornis (LACORDAIRE), A. carinata sp. n., A. loebli PACE, A. serieptunctata sp. n., A. armata sp. n., A. wrasei sp. n., A. megalops sp. n., A. formosana sp. n., A. densepunctata sp. n., and $A$. splendens JARRIGE. The identity of $A$. tingitana JARRIGE, whose holotype is lost, remains doubtful. The following synonymies, new combinations, and replacement name are established or confirmed, respectively: Calodera MANNERHEIM, $1830=$ Nasirema CASEY, 1893, syn. n.; Aleochara GrAVENHORST, $1802=$ Sorecocephala BernHAUER, 1902, syn. n.; Amarochara heterogaster CAMERON, $1939=$ A. simlaensis CAMERON, 1939, syn. n., = A. smetanai PACE, 1992, syn. n.; Calodera bonnairei FAUVEL, 1865, nomen protectum = Oxypoda glabriventris RYE, 1865, nomen oblitum; Dasygnypeta velata (ERICHSON, 1837) = Calodera flavipes MOTSCHULSKY, 1858 (previously a synonym of Amarochara forticornis); Alevonota japonica (CAMERON, 1933), comb. n. (from Amarocbara); Calodera caseyi nom. n., comb. n. (originally described as Nasirema humilis CASEY, 1893, previously attributed to Amarochara, and a secondary junior homonym of Calodera bumilis ERICHSON); Calodera parviceps (CASEY, 1893), comb. n. (originally in Nasirema, previously a presumed synonym of Amarachara umbrosa); Pseudocalea korbi (BERNHAUER, 1902), comb. n. (from Amarocbara); Aleochara (Ceranota) ocaleoides (BERNHAUER, 1902), comb. n. (from Amarochara); Aleochara (Ceranota) subtumida (HOCHHUTH, 1849) $=$ Calodera brunnea MOTSCHULSKY, 1860 (previously a synonym of Amarochara forticornis), $=$ Ocalea reitteri BERNHAUER, 1900, syn. n. Amarocbara flavicomis BERNHAUER, 1907 is excluded from Amarochara and attributed to the Athetini, but of doubtful generic affiliations. A neotype is designated for Calodera bonnairei FAUVEL. Lectotypes are designated for Calodera umbrosa ERICHSON, 1837, Amarochara heterogaster CAMERON, 1939, Nasirema inquilina CASEY, 1906, Oxypoda glabriventris RYE, 1865, lyyobates cribripennis MULSANT \& REY, 1875, and Ocalea reitteri BERNHAUER, 1900. The taxonomic history of Amarochara is outlined. Based on a phylogenetic analysis, the prevailing subgeneric concept is not adopted. As the species of zoogeographic regions other than the Holarctic have not yet been revised, however, the subgenera are not formally synonymized. Instead, the Holatctic species of the genus are attributed to five species groups. The (re-)descriptions of the genus, the species groups, and the species are complemented by a key and by figures of the mouthparts, primary and secondary sexual characters, and other distinguishing characters. The available biogeographic data are compiled and the distributions of several species are mapped. As can be inferred from field studies and collection data, species of Amarochara are univoltine, with reproduction taking place in spring and summer. Apparently, reproduction and hibernation take place in an unknown subterranean habitat. Ecological data are compiled and discussed, and the phenologies of the better-known species are figured. The genitalia of Pseudocalea korbi (BERNHAUER) and Aleochara ocaleoides (BERNHAUER) ate illustrated. 


\section{Keywords}

Coleoptera - Staphylinidae - Aleocharinae - Oxypodini - Amarochara - Nasirema - Calodera - Ocalea - Pseudocalea - Aleochara - Alevonota - Dasygnypeta - Sorecocephala - Holarctic region - Palaearctic region - Nearctic region - taxonomy - biogeography - ecology - life history - new species - new synonymy - new combination - neotype designation - lectotype designation

\section{Zusammenfassung}

Die Typen und weiteres Material der in der Holarktis vorkommenden Arten der Gattung Amarachara THOMSON werden revidiert. Von 25 als valid erkannten Arten werden 24 beschrieben bzw. redeskribiert:

A. umbrosa (ERIChson), A. beterogaster Cameron, A. sororcula CAMEron, A. inquilina (CASEY), A. femyesi BLATCHLEY, A. brevios sp. n., A. bonnairei (FAUVEL), A. cribripennis (MULSANT \& REY), A. siculifera sp. n., A. inermis sp. n., A. caeca sp. n., A. crassicornis (QUEDENFELDT), A. forticornis (LACORDARE), A. carinata sp. n., A. loebli PACE, A. seriepunctata sp. n., A. armata sp. n., A. urasei sp. n., A. megalops sp. n., A. formosana sp. n., $A$. densepunctata sp. n. und $A$. splendens JARrIGE. Die Identität von $A$. tingitana JARrige, deren Holotypus verschollen ist, bleibt ungeklärt. Die Revision ergab folgende Synonymien, Neukombinationen und Ersatznamen: Calodera MANNERHEIM, $1830=$ Nasirema CASEY, 1893, syn. n.; Aleochara GRAVENHORST, 1802 = Sorecocephala Bernhauer, 1902, syn. n.; Amarochara beterogaster CAMERON, $1939=$ A. simlaensis CAMERON, 1939, syn. n., = A. smetanai PACE, 1992, syn. n.; Calodera bonnairei FAUVEL, 1865, nomen protectum = Oxypoda glabriventris RYE, 1865, nomen oblitum; Dasygnypeta velata (ERICHSON, 1837) Calodera flavipes MOTSCHULSKY, 1858 (zuvor Synonym von Amarochara forticornis); Alevonota japonica (CAMERON, 1933), comb. n. (ursprünglich Amarochara); Calodera caseyi nom. n., comb. n. (ursprünglich als Nasirema bumilis CASEY, 1893 beschrieben und später Amarochara zugeordnet, sekundäres jüngeres Synonym von Calodera bumilis ERICHSON); Calodera parviceps (CASEY, 1893), comb. n. (ursprünglich als Nasirema beschrieben, später als Synonym von Amarocbara umbrosa vermutet); Pseudocalea korbi (BERNHAUER, 1902), comb. n. (ursprünglich Amarochara); Aleochara (Ceranotc) ocaleoides (BERNHAUER, 1902), comb. n. (ursprünglich Amarocbara); Aleocbara (Ceranota) subtumida (HOCHHUTH, 1849) - Calodera brunnea MOTSCHULSKY, 1860 (vorher Synonym von Amarochara forticornis), = Ocalea reitteri BERNHAUER, 1900, syn. $\mathrm{n}$. Amarochara flavicornis BERNHAUER, 1907 wird in die Tribus Athetini transferiert, ihre Gattungszugehörigkeit ist jedoch zweifelhaft. Für Calodera bonnairei FAUVEL wird ein Neotypus designiert. Lectotypen werden designiert für Calodera umbrosa ERICHSON, 1837, Amarochara heterogaster CAMERON, 1939, Nasirema inquilina CASEY, 1906, Oxypoda glabriventris RYE, 1865, Ibobates cribripennis MULSANT \& REY, 1875 und Ocalea reitteri BERNHAUER, 1900. In einem historischen Überblick werden die taxonomischen Entwicklungen zur Gattung Amarochara zusammengefasst. Auf der Grundlage phylogenetischer Untersuchungen und Schlussfolgerungen wird die bestehende Untergattungssystematik nicht übernommen. Da aber die Arten anderer zoogeographischer Regionen bisher nicht revidiert wurden, werden die Subgenera nicht formal synonymisiert. Statt dessen werden die holarktischen Vertreter der Gattung 5 Artengruppen zugeordnet. Die Beschreibungen der Gattung, der Attengruppen sowie der Arten werden durch eine Bestimmungstabelle und durch Abbildungen der Mundteile, der primären und sekundären Geschlechtsmerkmale sowie weiterer Unterscheidungsmerkmale ergänzt. Die verfügbaren biogeographischen Daten werden zusammengefasst; für eine Reihe von Arten werden Verbreitungskarten vorgelegt. Ergebnisse von Freilanduntersuchungen und Sammlungsdaten deuten darauf hin, dass die holarktischen Amarochara-Arten univoltin sind und sich im Frühjahr und Sommer fortpflanzen. Reproduktion und Überwinterung finden offenbar in einem bisher unbekannten unterirdischen Habitat statt. Die verfügbaren ökologischen Daten werden zusammengefasst und diskutiert. Die Phänologien einiger besser bekannter Arten werden in Form von Diagrammen illustriert. Die Sexualmerkmale zweier in andere Gattungen kombinierter Arten, Pseudocalea korbi (BERNHAUER) und Aleochara ocaleoides (BERNHAUER), werden abgebildet. 


\section{Table of contents}

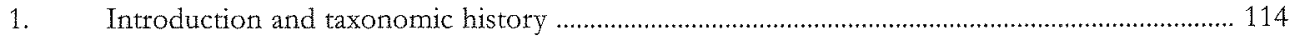

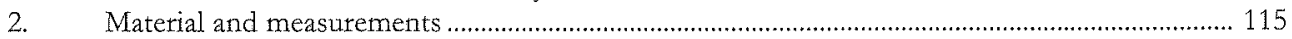

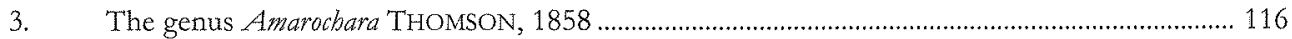

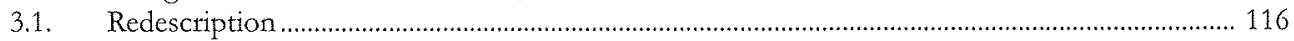

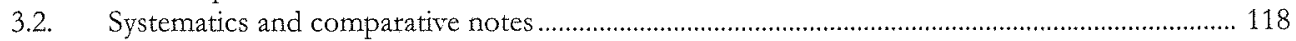

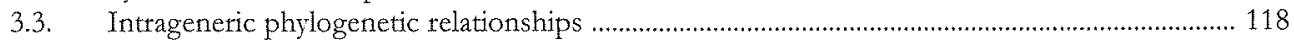

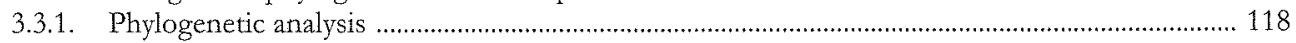

3.3.1.1. Discussion of character states and polarities ........................................................................ 119

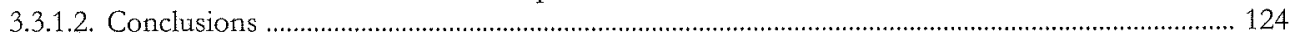

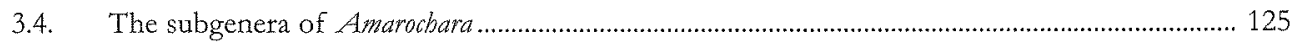

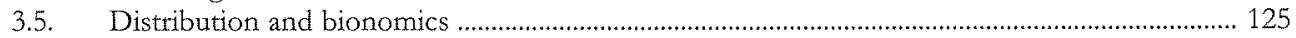

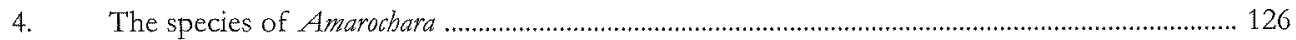

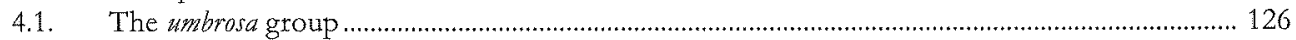

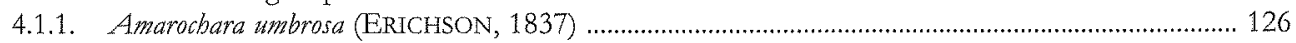

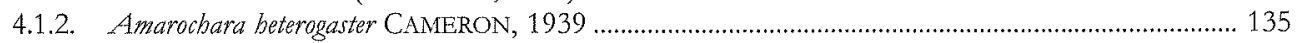

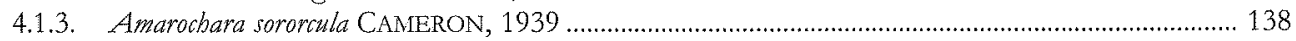

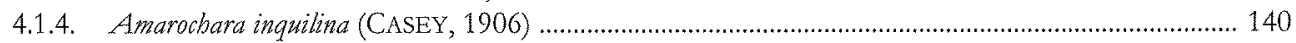

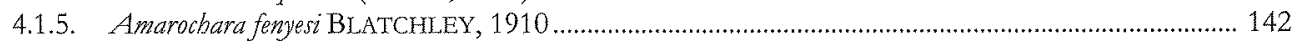

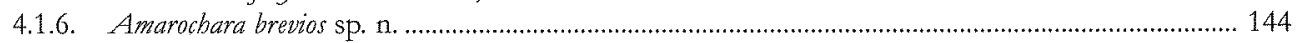

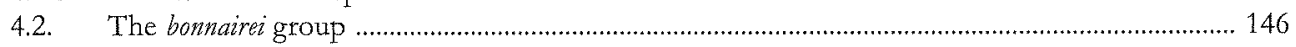

4.2.1. Amarochara bomnairei (FAUVEL, 1865), nomen protectum ......................................................... 147

4.2.2. Amarocbara cribripennis (MULSANT \& REY, 1875) …............................................................. 152

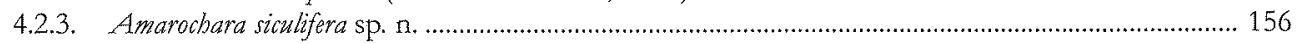

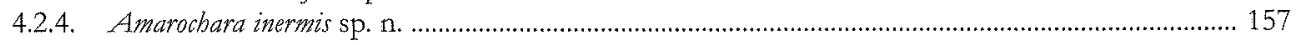

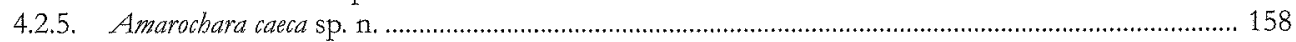

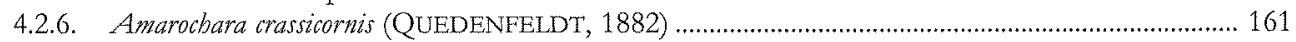

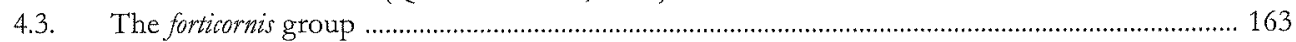

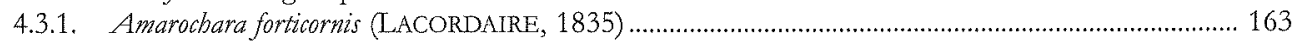

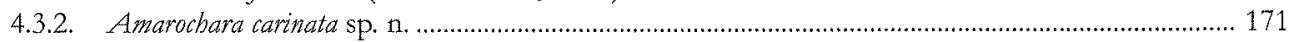

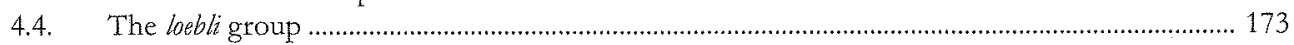

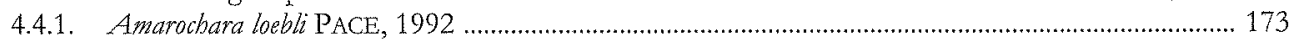

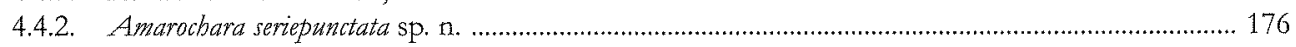

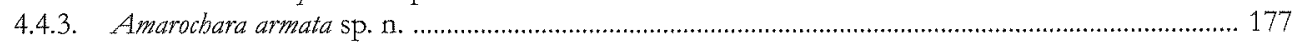

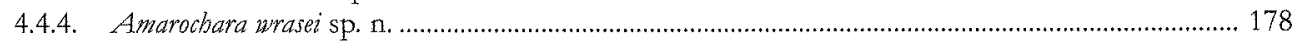

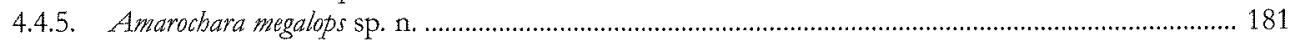

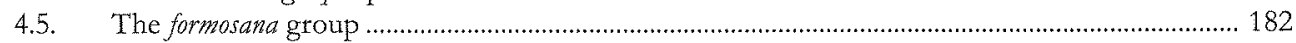

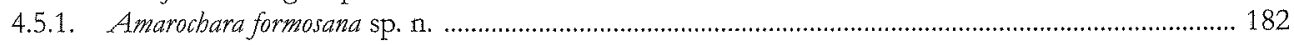

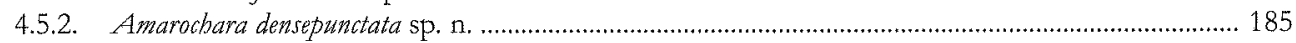

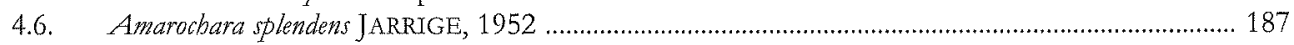

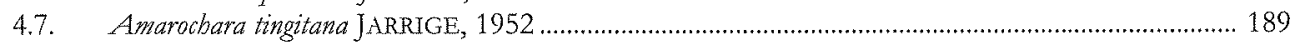

5. Key to the Amarochara species of the Holarctic region .............................................................. 189

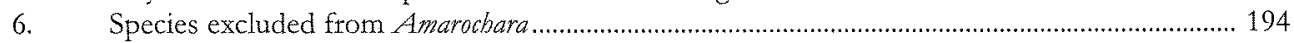

6.1. Dasygnypeta velata (ERICHSON, 1837) …….............................................................................. 194

6.2. Alevonota japonica (CAMERoN, 1933), comb. n. ..................................................................... 195

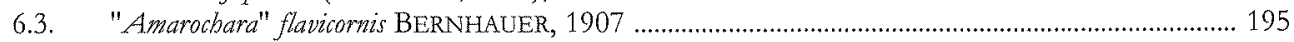

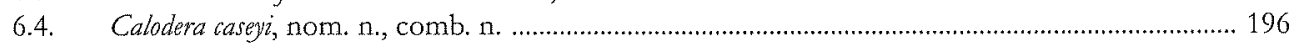

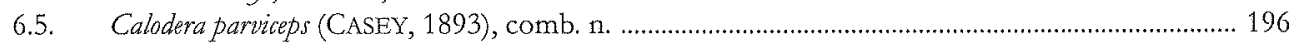

6.6. Pseudocalea korbi (BERNHAUER, 1902), comb. n. ..................................................................... 197

6.7. Aleochara ocaleoides (BERNHAUER, 1902), comb. n. ................................................................... 199

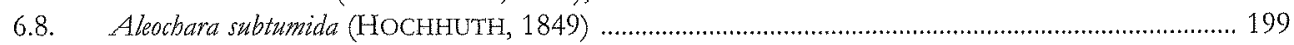

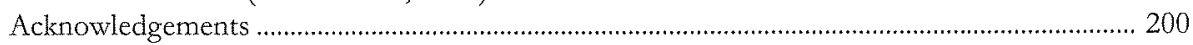

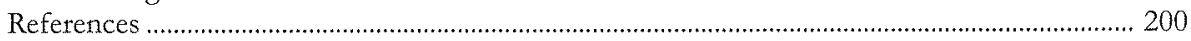




\section{Introduction and taxonomic history}

When THOMSON (1858) described Amarochara in a key to the Swedish subfamilies, tribes and genera of Staphylinidae, the genus included only the type species $A$. umbrosa (ERICHSON). MULSANT \& REY (1875) gave a detailed redescription of both the genus and the species and described Mniobates, a subgenus of Ilyobates KRAATZ, 1856, to which they assigned Bolitochara forticornis LACORDAIRE (later to be designated type species of the subgenus), Calodera bonnairei FAUVEL, and Ilyobates cribripennis. The latter name was made available in the same work, although the authors, apparently unaware that it was an inlitteris name, attributed it to Fauvel. FOWLER (1888) synonymized Amarochara by transferring C. umbrosa ERICHSON to Calodera again and, with some doubt regarding the priority, regarded Ilyobates bonnairei (FAUVEL, 1865) as a junior synonym of Ilyobates glabriventris (RYE, 1865), which had originally been described in the genus Oxypoda MANNERHEIM, 1830. GANGLBAUER (1895) reversed this synonymy, reinstated Amarochara as a distinct genus, transferred Mniobates MULSANT \& REY with the sole representative $A$. forticornis (LACORDAIRE) to Amarochara as a subgenus, and established the new subgenus Lasiochara for A. bonnairei (FAUVEL), which had previously been in Ilyobates. He distinguished the subgenera based on the presence of an anterior impression on the abdominal tergum VI and a carina on the mesosternum (Mniobates), as well as on the presence and absence of genal carinae (Lasiocbara and Amarochara s. str., respectively). BERNHAUER (1902) maintained this concept and presented a key to the three subgenera and seven Palaearctic species known at that time, with one species (A. umbrosa) in the nominal subgenus, four species (A. bonnairei, A. cribripennis, A. crassicornis (QUEDENFELDT), and the new A. ocaleoides) in Lasiochara, and two species (A. forticornis and the new A. korbi) in Mniobates.

BERNHAUER \& SCHEERPELTZ (1926) listed 14 valid species, 5 synonyms, and 4 subgenera of Amarochara, 1 species from the Ethiopian, 2 species and a subgenus from the Neotropical, 3 species and a synonym from the Nearctic, and the remainder from the Palaearctic region. Three of the Nearctic names (2 valid species and the synonym) had been attributed to the genus Nasirema CASEY, 1893 by CASEY $(1893,1906)$ (see also FENYES (1920)), which was regarded as a junior synonym of Amarochara by BERNHAUER \& SCHEERPELTZ (1926). Since the publication of this catalogue the subgeneric concept has remained the same and 19 further species have been described, two from the Western Palaearctic (A. tingitana JARRIGE, 1952 and A. splendens JARRIGE, 1952), six from the Eastern Palaearctic (A. simlaensis CAMERON, 1939, A. beterogaster CAMERON, 1939, A. sororcula CAneron, 1939, A. japonica CAnieron, 1933, A. loebli PACE, 1992, and A. smetanai PACE, 1992), eight from the Ethiopian, and three species from the Oriental region.

The present revision was initiated especially by the difficulties encountered when attempting to identify Amarochara material from the south of the Western Palaearctic region. With the exception of some recently described species from the Eastern Palaearctic, the Oriental, and the Ethiopian region, the sexual characters have not been studied and the systematic status (including the prevailing subgeneric concept) has not been reexamined. Since one of the North America names in the genus has been treated as a junior synonym of a species described from the Western Palaearctic region, this first revisionary step covers both the Palaearctic and the Nearctic region. 
In the course of the revision, a loan of the types of $A$. tingitana JARRIGE and $A$. splendens JARRIGE was attempted, but repeated requests remained unanswered. As an agreed deadline had to be met, the revision was submitted and the two taxa were treated as doubtful species. It was by chance that several weeks after submission of the manuscript Alexey Solodornikov, St. Petersburg, informed me that he was planning to visit the MNHNP and kindly offered to use this opportunity to try and initiate a loan of the holotypes in question. His efforts were successful, so that it was possible to clarify at least the identity of $A$. splendens. Unfortunately, however, the holotype of $A$. tingitana was lost, so that an interpretation of that species will have to wait until material matching the description becomes available and a neotype can be designated. In order to avoid a separate supplement on the identity of $A$. splendens and because the date for the publication of volume 51 was postponed due to a reorganisation of the publishing procedures, the editorial staff of the journal kindly offered to incorporate not only the changes and additions resulting from the unexpected availability of the type of that species, but also some further material that had been studied in the meantime. In view of deadlines to be met, however, A. splendens could not be considered in chapter 3, its redescription (including the figures) is added as a separate section at the end of chapter 4 (rather than in section 4.2), and the additional material (marked with an asterisk) is accounted for neither in the maps nor in the diagrams.

\section{Material and measurements}

Types and additional material from the following institutions and private collections were examined:

BMNH The Natural History Museum, London (M. BRENDELL)

DEI Deutsches Entomologisches Institut, Eberswalde (L. ZERCHE)

FIS Forschungsinstitut Senckenberg, Frankfurt a. M. (A. VESMANIS)

FMNH Field Museum of Natural History, Chicago (P. P. PARRILLO)

HNHM Hungarian Natural History Museum, Budapest (O. MerkL)

IRSNB Institut Royal des Sciences Naturelles de Belgique, Bruxelles (D. DRUGMAND)

MHNG Nuséum d'Histoire Naturelle, Genève (G. Cuccodoro)

MHNL Muséum d'Histoire Naturelle, Lyon (J. CLARY; H. LABrique)

MNHUB Museutn für Naturkunde der Humboldt-Universität, Berlin (M. UHLIG)

MNHNP Muséum National d'Histoire Naturelle, Paris (N. BERTI)

NHMB Naturhistorisches Museum Basel (D. BURCKHARDT; E. SPRECHER)

NHMW Naturhistorisches Museum Wien (H. SCHLlHAmMER)

NMB Naturkunde-Museum Bielefeld (K. RENNER)

NME Naturkundemuseum Erfurt (M. HARTMANN; J. WILLERS)

NMP Národní Muzeum v Praze (J. JeLíneK)

PU Purdue University, West Lafayette (A. PROvONSHA)

SMNO Staatliches Museum für Naturkunde und Vorgeschichte Oldenburg (A. ROSE)

SMNS Staatliches Museum für Naturkunde in Stuttgart (W. SCHAWALLER; K. WOLF-SCHWENNINGER)

SMTD Staatliches Museum für Tierkunde, Dresden (O. J I GGER)

TLMFI Tiroler Landesmuseum Ferdinandeum, Innsbruck (M. KAHLEN) 


$\begin{array}{ll}\text { UKNHM } & \text { University of Kansas Natural History Museum, Lawrence (J. S. ASHE; R. W. BROOKS) } \\ \text { USNM } & \text { U. S. National Museum of Natural History, Washington (via J. S. ASHE, Kansas) } \\ \text { ZMMU } & \text { Zoological Museum Moscow Lomonosor State University (N. NIKITSKY) } \\ \text { cAss } & \text { author's private collection } \\ \text { cFel } & \text { private collection B. FELDMANN, Münster } \\ \text { cKöh } & \text { private collection F. KÖHLER, Bornheim } \\ \text { cOwe } & \text { private collection J. A. OWEN, Epsom } \\ \text { cRen } & \text { private collection K. RENNER, Bielefeld } \\ \text { cSch } & \text { private collection M. SCHULKE, Berlin } \\ \text { cTro } & \text { private collection M. TRONQUET, Molitgles-Bains } \\ \text { cVog } & \text { private collection J. VOGEL, Görlitz } \\ \text { cWun } & \text { private collection P. WUNDERLE, Mönchengladbach } \\ \text { cZan } & \text { private collection A. ZANETT, Verona }\end{array}$

Head length was measured from the anterior margin of the clypeus to the posterior margin of the head, elytral length (at suture) from the apex of the scutellum to the posterior margin of the elytra.

\section{The genus Amarochara THOMSON, 1858}

Amarochara THOMSON, 1858: 32.

Type species: Calodera umbrosa ERICHSON

\subsection{Redescription}

Species of small to intermediate size and of slender build.

Microsculpture of head and pronotum absent or, if present, usually shallow and composed of meshes of variable length and orientation, interstices of elytra in the vast majority of species without distinct microsculpture; abdomen very shining, anterior segments usually without microsculpture, posterior segments often with weakly pronounced transverse microsculpture. Punctures on head and pronotum of variable density, moderately to extremely fine; puncturation on elytra and abdomen variable. Pubescence of forebody in most species relatively long and \pm suberect, rarely short and/or decumbent.

Head subquadrate, subcircular, or of \pm ovoid shape, slightly or distinctly narrower than pronotum; posteriorly with or without distinct neck; dorsal pubescence in lateral areas directed anteriad and in median area \pm transversely mediad or diagonally antero-mediad. Eyes moderately to very large, though in most species shorter than the postgenae in dorsal view, weakly to distinctly projecting from lateral outline of head. Genal carina present or reduced to various degrees. Gular sutures widely separated. Dorsal area of vertex not distinctly impressed.

Antennae relatively long and massive; antennomere I dorso-apically distinctly emarginate, this emargination basad extending into a \pm pronounced furrow (which accommodates the pedicel when the antenna is fully bent backwards); antennomeres I-III oblong, I 
and II subequal in length; antennomere III coniform, somewhat shorter than I and II or of approximately equal length; IV and V strongly transverse, VI-X more or less transverse (but less so than IV and V), at most only indistinctly increasing in width apically; XI approximately as long as the combined length of IX and X; pubescence of I-III relatively sparse and moderately short, that of $I V$ and $V$ dense and short, and that of VI-XI extremely short, fine, and very dense (Figs. 5, 37, 50, 57, 66, 69, 77, 90, 110).

Penultimate joint of maxillary palpi very long and slender, apical joint long, slender, and weakly coniform (Figs. 1, 18, 34, 49, 61, 76, 89, 109); mandibles apically distinctly bent and acute, right mandible without distinct molar tooth, faintly serrate or smooth in dorsal molar region (Fig. 4); ligula weakly to distinctly bifid, apically often with a fine and short, sometimes barely noticeable sensillum (Figs. 2, 17, 25, 33, 48, 75, 88, 108, 113); mentum with few long setae (Fig. 2); labrum anteriorly broadly membranous and with two obtuse median projections $(3,16)$.

Pronotum weakly to moderately transverse; posterior angles obtuse, usually weakly marked, sometimes more or less obsolete; maximal width in, or a short distance anterior to middle; surface distinctly convex in cross-section; pubescence along midline directed cau$\mathrm{dad}$, in lateral areas \pm diagonally latero-caudad, and near posterior margin \pm transversely laterad. Hypomera distinctly visible in lateral view. Prosternum with median carina.

Elytra as wide as or slightly wider than pronotum and at suture slightly to distinctly shorter than pronotum; hind margin near posterior angles strongly or indistinctly sinuate; hind wings fully developed; mesosternum with ( \pm reduced) or without median carina; mesosternal process long, reaching halfway between mesocoxae, apically acute, tounded, or distinctly truncate; mesocoxal cavities delimited from metasternum by carina.

Legs long and slender; first metatarsomere very long and slender, at least as long as the combined length of the two following tarsomeres (Figs. 35, 67, 70).

Abdominal terga III-V with \pm deep, tergum VI with weaker or without anterior impressions; sterna III-V or III-VI anteriorly constricted; tergum VIII posteriorly convex and with relatively long thin setae (Figs. 11, 13); tergum $X$ anteriorly often with \pm long and slender extension, with numerous stout setae, which are longer posteriorly than anteriorly, in posterior median area in most species without setae, posterior margin with membranous fringe (Figs. 6, 20, 27, 36, 43, 56, 79, 91, 111, 114).

$0^{*}$ : sternum VIII posteriorly weakly to moderately convex with a row of moderately long and a row of longer thin setae (Fig. 12); median lobe of aedeagus with almost straight ventral process (lateral view), at base of ventral process often with lateral and/ or median folds, and with weakly pronounced crista apicalis; internal sac with moderately to very long internal tube, and with weakly to distinctly sclerotized additional structures; apical lobe of paramere conspicuously small and short, usually \pm coniform (e. g. Fig. 7), sometimes modified (flattened, with torsion, or even transverse) (Figs. 84, 85, 95, 98), with two moderately long subapical and two short apical setae. For illustrations of the parameres of $A$. umbrosa (plesiomorphic condition) and $A$. armata sp. n. (derived condition) see SEEVERS (1978) and Fig. 94, respectively.

$q$ : sternum VIII similar to that of $\sigma^{*}$, posteriorly usually less strongly convex, its posterior margin with slender, weakly modified or unmodified setae, and without micropubescence (Fig. 14); spermatheca of distinctive morphology, capsule large and bulbous, duct relatively short and \pm straight (e. g. Figs. 10, 24, 28, 32, 42, 47); general shape of spermatheca somewhat resembling that of Piochardia HEYDEN of the Aleocharini. 


\subsection{Systematics and comparative notes}

Based on morphological characters, Amarochara is correctly placed in the Oxypodini. From other genera of this tribe, it is distinguished especially by the morphology of the antennae, by the morphology of the median lobe of the aedeagus, of the apical lobe of the paramere, and of the spermatheca, and from many genera also by the absence of micropubescence and of distinctly modified setae on the posterior margin of the of sternum VIII. A closer relationship to Calodera MANNERHEIM, 1830 and Ilyobates KRAATZ, 1856 , as presumed by previous authors because of the similar external appearance (slender body, weakly transverse pronotum, relatively long and slender legs) and especially the stout antennae, seems rather unlikely. Amarochara is separated from these genera by numerous significant characters, especially by the morphology of the mouthparts (ligula, chaetotaxy of the labrum, slender maxillary palpi) and of the antennae (dorso-apical furrow on antennomere I, strongly transverse antennomere IV and $V$, shape and pubescence of antennomeres VI-X), by the shape and chaetotaxy of tergum X, the shape and internal structures of the median lobe of the aedeagus, the short apical lobe of the paramere with relatively short setae, and by the characteristic morphology of the spermatheca. In addition, Amarochara is of almost global distribution, whereas Ibobates is Western Palaearctic and Calodera probably Holarctic. (Several species of Calodera have been described also from other biogeographic region, but those species that I have examined refer to other genera; the same is probably true of those taxa that have not been reexamined.) The adelphotaxon of Amarochara is unknown. Among the betterknown genera of Palaearctic Oxypodini, there is no likely candidate.

\subsection{Intrageneric phylogenetic relationships}

\subsubsection{Phylogenetic analysis}

In order to assess the intrageneric phylogenetic affiliations, morphological and biogeographic data were compiled and transformed into a data matrix. As the adelphotaxon of Amarachara is unknown, several representatives of presumably more closely related oxypodine genera were selected to determine character polarity: Calodera nigrita MANNERHEIM, C. rufescens KRAATZ, Euryalea decumana (ERICHSON), E. murina (ERICHSON), Ilyobates bennetti DONISTHORPE, and Ocalea picata (STEPHENS). As more distant outgroup, a species of Zoosetha was chosen: Z. rufescens (KRAATZ). Whenever in doubt regarding character polarity, the corresponding character states in other aleocharine tribes (Falagriini, Athetini) were considered in the character discussion, but are not listed in the data matrix below.

For illustrations and descriptions of the taxa here selected as outgroups see AssinG (1996, 1998, 1999) and ASsing \& WUNDERLE (1997, 1998). 


\subsubsection{Discussion of character states and polarities}

Eyes: large, in dorsal view approximately as long as postgenae or nearly so (0); distinctly shorter than postgenae (1).

Head: lateral margins posteriorly smoothly rounded, posterior angles weakly marked of obsolete (0); posterior angles abruptly rounded (1).

Head: microsculpture obsolete or nearly so $(0)$; microsculpture \pm distinct (1).

Head: posteriorly not distinctly constricted $(0)$; with distinct neck (1).

Head: genal carinae pronounced (0); present, but very fine (1); reduced to short rudiments (2); absent (3).

Antenna: antennomere I dorso-apically without furrow and with weak emargination (0); antennomere I dorso-apically with furrow and with pronounced emargination (1).

Antenna: not massive and antennomeres IV-X not transverse (0); massive and antennomeres IV-X transverse (1). The massive antennae in Amarochara, Iyobates, Calodera, and Zoosetha are very unlikely to be homologous. At least in Ilyobates and Zoosetha, they are of different morphology in many other respects.

Antenna: antennomeres VI-X distinctly transverse, but moderately wide, $<1.5$ times as wide as antennomere I (0); antennomeres VI-X massive, $>1.5$ times as wide as antennomere I (1).

Antenna: massive and relatively short, when turned backwards only slightly extending beyond pronotal hind matgin (0); massive and long, distinctly extending beyond pronotal hind margin, reaching posterior margin of elytra or nearly so (1). The long antennae in most of the outgroup taxa are unlikely to be homologous to those in Amarochara and primarily due to the long and slender antennomeres IV-X; this is particularly true of the antennae in Euryalea and Ocalea. Long antennae are evidently homoplastic in several species groups of Amarochara.

Antenna: connecting joints between antennomeres of normal width (0); connecting joints broad (1).

Antenna: dorso-apical furrow of antennomere I weakly pronounced, usually about half the length of antennomere (0); dorso-apical furrow pronounced, almost extending over whole length of antennomere $I$ (1). This character is subject to considerable intraspecific variation and consequently attributed only little significance.

Antenna: antennomere III relatively long and slender, approximately as long as antennomere II or nearly so (0); antennomere III distinctly coniform and clearly shorter than antennomere II. The polarity of this characters is doubtful.

Antenna: antennometes IV and V modexately transverse (0); extremely transverse, disc-shaped (1).

4 Maxillary palpus: third joint moderately slender, $2-3$ times as long as broad (0); third joint very slender, more than 3 times as long as broad (1). The derived condition of this character in some species of Amarochara on the one hand and in Ocalea and Euryalea on the other hand is very unlikely to be homologous.

Ligula: distinctly bifid (0); only apically bifid (1); long and slender, not bifid (2). The polarity of character states 0 and 1 is doubtful.

Right mandible: with tooth (0); without or with very weak tooth (1).

Forebody: with fine to moderately coarse puncturation $(0)$; with conspicuously coarse puncturation (1).

Pubescence of forebody: relatively long and semi-erect (0); short and dense (1). The derived condition in some Amarochara and $C$, nigrita is without doubt homoplastic.

Pronotum: microsculpture obsolete or nearly so (0); microsculpture present (1). 
20 Pronotum: puncturation moderately sparse (0); puncturation sparse (1); puncturation conspicuously dense (2). The similar character code for some Amarocbara, Ilyobates, E. murina, Calodera spp., and $Z$. rufescens is without doubt homoplastic and at least in part a morphological adaptation to humid habitats; see also character 17. The intermediate condition is hypothesized to be plesiomorphic, the two extreme conditions are likely to be derived.

21 Pronotum: posterior angles well-defined (0); posterior angles obsolete or nearly so (1). This character is subject to some intraspecific variation and attributed little phylogenetic significance.

22 Pronotum small in relation to head, less than 1.3 times as wide as head (0); pronotum large, at least 1.3 time as wide as head (1). This character is of limited significance for assessing phylogenetic relationships, since it is subject to some intraspecific variation and several species are of intermediate condition.

23 Elytra: microsculpture present (0); microsculpture absent (1).

24 Elytta: posterior margin weakly sinuate near posterior angles $(0)$; not or only indistinctly sinuate near posterior angles (1); strongly sinuate (2). The character codes do not reflect an evolutionary trend; the intermediate condition is apparently plesiomorphic.

25 Mesosternum: mesosternal carina absent (0); present (1). The polarity of this character is uncertain. It is known to be subject to intrageneric variation also in other aleocharine genera (e. g. Aleochara GRAVENHORST and Acrotona THOMSON).

26 Mesosternum: mesosternal process apically acute (0); process apically narrowly truncate (1); process broadly truncate (2).

27 Metatarsus: metatarsomere I relatively short, distinctly shorter than the combined length of the three following tarsomeres (0); metatarsomere I approximately as long as the combined length of the three following tarsomeres or longer (1). This character is subject to considerable intraspecific and in-group variation and therefore attributed little phylogenetic significance.

28 Abdominal terga III-V: anterior impressions moderately deep (0); impressions very deep (1); impressions very shallow (2).

29 Abdominal tergum VI: anterior impression shallow or obsolete (0); impression deep (1). The occutrence of species with the derived condition in Amarochara, Calodera, and Euryalea reflects a parallel evolutionary trend in these genera. There is, however, some doubt if the polarity here assumed also applies to Calodera.

30 Abdominal terga III-VI: puncturation anteriotly moderately coarse (0); puncturation anteriorly conspicuously coarse and dense (1); puncturation anteriorly fine (2). The derived character state 1 in some species of the umbrosa group and in other species groups of Amarochara is apparently the result of parallel evolution.

31 Abdominal terga III-VI: puncturation of posterior half moderately dense (0); very dense, as dense as in anterior impressions or nearly so (1); puncturation sparse (2).

32 Abdominal terga III-VI: puncturation of posterior half moderately coatse (0); very fine (1); coarse (2).

33 Abdominal terga III VT: without transverse series of coarse punctures in posterior half (0); with transverse series of punctures in posterior half (1).

$34 \quad$ o sternum VIII: posteriotly with micropubescence (0); without micropubescence at posterior margin (1). The polarity assumed here is based on the observation that the presence of micropubescence is the usual condition found in many Aleocharinae, not only in Oxypodini, but also in Athetini, Falagriini, etc.

35 o sternum VIII: marginal setae at posterio margin distinctly modified (i. e. very stout) (0); weakly modified (1). It can be inferred from the presence of distinctly modified setae in most Oxypodini and in several other aleocharine tribes that the weakly modified setae are apparently the result of a reduction and consequently represent the derived condition.

36 Abdominal tergum $\mathrm{X}$ : anteriorly weakly elongated (0); anteriorly distinctly elongated (1). 
Aedeagus: median lobe in lateral riew not narrow or compressed $(0)$; median lobe flat, in lateral view \pm compressed (1).

38 Aedeagus: ventral process of median lobe distinctly bent ventrad (0); ventral process straight or nearly so (1).

39 Aedeagus: ventral process fused with dorsal part of main body (0); ventral process separated for most of its length (1). The derived condition in some Amarocbara on the one hand and in Euryalea on the other hand is clearly not homologous.

40 Aedeagus: ventral process long and slender, $>2.5$ times as long as wide (0); ventral process broad and short (1); ventral process conspicuously short, much shorter than basal part of median lobe (2).

41 Aedeagus: internal structures not or weakly sclerotized (0); internal sac with pairs of distinctly sclerotized structures (1). The derived conditions in some Amarochara, Ilyobates, Ocalea, and Calodera are clearly not homologous. This is apparently also true of the internal structures found in $A$. forticornis and in some Eastern Palaearctic Amarochara; they are of completely different morphology.

Aedeagus: median carina of ventral process absent or weakly pronounced (0); ventral process at least basally with distinct median carina (1).

Aedeagus: Ventral process without lateral carinae (0); at least at base with lateral carinae (1).

Aedeagus: without process at base of ventral process (0); with such process (1).

Aedeagus: apical part of internal tube short (0); moderately long (1); very long (2).

Aedeagus: basal part of internal tube short (0); moderately long (1); very long (2).

Aedeagus: crista apicalis distinct (0); crista apicalis reduced (1).

Aedeagus: crista proximalis distinct (0); crista proximalis reduced (1).

49 Aedeagus: apical lobe of paramere long and slender, $>3$ times as long as wide and with long setae (0); apical lobe of reduced length and with shorter setae (1).

Aedeagus: apical lobe of paramere slender, approximately twice as long as wide (0); coniform or otherwise modified (1).

Aedeagus: apical lobe of paramere of \pm coniform shape and oval in cross-section (0); apical lobe of paramere not coniform, distinctly flattened in cross-section (1).

Aedeagus: apical lobe of paramere flattened, at least weakly oblong (0); apical lobe of paramere distinctly transverse (1).

53 Spermatheca: capsule slender, of ovoid shape (0); capsule globulous (1). The similar conditions in Amarocbara on the one hand and in I. bennetti on the other are cleatly homoplastic. In most species of Ilyobates the plesiomorphic condition is realized.

Spermatheca: capsule not or only weakly separated from duct (0); capsule distinctly separated from duct (1).

Spermatheca: duct long, coiled or U-shaped (0); duct short and \pm straight. The similar conditions in Amarocbara on the one hand and in $I$. bennetti on the other are clearly homoplastic. In most species of Ilyobates the plesiomorphic condition is realized.

Spermatheca: without separate tube between duct and capsule (0); capsule connected to duct by separate short tube (see Fig. 112).

Spermatheca: capsule apically not constricted (0); capsule apically constricted (1).

Distribution: Western Palaearctic (1); Eastern Palaearctic (2); Nearctic (3). 
Tab. 1: Character matrix. Explanation of abbreviations: $\mathrm{t}=$ transitional $(0-1) ; ?=$ unknown; - = logically impossible; $\mathrm{i}=$ irrelevant.

\begin{tabular}{|c|c|c|c|c|c|c|c|c|c|c|c|c|c|c|c|c|c|c|c|c|}
\hline Character & 1 & 2 & 3 & 4 & 5 & 6 & 7 & 8 & 9 & 10 & 11 & 12 & 13 & 14 & 15 & 16 & 17 & 18 & 19 & 20 \\
\hline sororcula & 0 & 1 & 0 & 0 & 1 & 1 & 1 & 0 & 0 & 0 & 1 & 1 & 0 & 0 & 0 & 1 & 0 & 1 & 0 & 0 \\
\hline heterogaster & 0 & $t$ & 0 & 0 & 2 & 1 & 1 & 1 & 0 & 0 & 0 & 1 & 0 & 0 & 0 & 1 & 0 & 1 & 0 & 0 \\
\hline tumbrosa & 0 & 1 & 0 & 0 & 3 & 1 & 1 & 0 & 0 & 0 & 1 & 1 & 0 & 0 & 0 & 1 & 0 & 0 & 0 & 0 \\
\hline inquilina & 0 & $t$ & 0 & 0 & 3 & 1 & 1 & 1 & 0 & 0 & 1 & 1 & 0 & 0 & 0 & 1 & 0 & 0 & 0 & 0 \\
\hline brevios & 0 & 1 & 0 & 0 & 3 & 1 & 1 & 1 & 0 & 0 & 0 & 1 & 0 & 0 & 0 & 1 & 0 & 0 & 0 & 0 \\
\hline fenvesi & 0 & 1 & 0 & 0 & 3 & 1 & 1 & 1 & 0 & 0 & 1 & 1 & 0 & 1 & $\theta$ & 1 & 0 & 0 & 0 & 0 \\
\hline bonmairet & 1 & 0 & 1 & 0 & 1 & 1 & 1 & 1 & 0 & 0 & 0 & 1 & 0 & 1 & 0 & 1 & 0 & 0 & 1 & 1 \\
\hline cribripennis & 1 & 0 & 0 & 0 & 1 & 1 & 1 & 1 & 1 & 0 & 0 & 1 & 0 & 1 & 0 & 1 & 0 & 0 & 0 & 1 \\
\hline siculffera & 1 & 0 & 0 & 0 & 1 & 1 & 1 & 1 & 1 & 0 & 0 & 0 & 0 & 1 & 0 & 1 & 0 & 0 & 0 & 1 \\
\hline inermis & 1 & 0 & 0 & 0 & 1 & 1 & 1 & 1 & 1 & 0 & 0 & 0 & 0 & 1 & 0 & 1 & 0 & 0 & 0 & 1 \\
\hline caeca & 0 & 0 & 1 & 0 & 1 & 1 & 1 & 1 & 1 & 0 & 0 & 0 & 0 & 1 & 0 & 1 & 0 & 0 & 1 & 1 \\
\hline crassicomis & 0 & 0 & 1 & 0 & 0 & 1 & 1 & 1 & 1 & 0 & 1 & 0 & 0 & 1 & 0 & 1 & 0 & 0 & 1 & 1 \\
\hline carinata & 1 & 0 & 0 & 0 & 0 & 1 & 1 & 1 & 1 & 0 & 1 & 0 & 0 & 1 & 0 & 1 & 0 & 0 & 0 & 1 \\
\hline forticornis & 1 & 0 & 0 & 0 & 0 & 1 & 1 & 1 & 1 & 0 & $\mathrm{t}$ & 0 & 0 & 1 & 0 & 1 & 0 & 0 & 0 & 1 \\
\hline loebli & 0 & 0 & 0 & 1 & 3 & 1 & 1 & 1 & 0 & 1 & 0 & 1 & 0 & 1 & 1 & 1 & $\theta$ & 0 & 0 & 1 \\
\hline seriepunctata & 0 & 0 & 0 & 1 & 3 & 1 & 1 & 1 & 0 & 1 & 0 & 1 & 0 & 1 & 1 & 1 & 0 & 0 & 0 & 1 \\
\hline armata & 0 & 0 & 0 & 1 & 3 & 1 & 1 & 1 & 1 & 1 & 0 & 1 & 0 & 1 & 1 & 1 & 0 & 0 & 0 & 1 \\
\hline wrasei & 0 & 0 & 0 & 1 & 3 & 1 & 1 & 1 & 0 & 1 & 1 & 1 & 1 & 1 & 1 & 1 & 0 & 0 & 0 & 1 \\
\hline nesalops & 0 & 0 & 0 & 1 & 3 & 1 & 1 & 1 & 1 & 1 & 1 & 1 & 0 & 1 & 1 & 1 & 0 & 0 & 0 & 1 \\
\hline formosana & 0 & 1 & 1 & 0 & 1 & 1 & 1 & 1 & 0 & 0 & 1 & 1 & 0 & 1 & 1 & 1 & 0 & 1 & 1 & 2 \\
\hline densepunctata & 0 & 0 & 1 & 0 & 0 & 1 & 1 & 1 & 1 & 0 & 1 & 1 & 0 & 1 & 1 & 1 & 0 & 1 & 0 & 0 \\
\hline E. dectumanu & 0 & 1 & 0 & 0 & 0 & 0 & 0 & - & - & 0 & - & 0 & - & 1 & 1 & 0 & 0 & 0 & 0 & 0 \\
\hline E. murina & 0 & 1 & 0 & 0 & 0 & 0 & 0 & - & - & 0 & 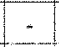 & 0 & $1-$ & 1 & 1 & 0 & 0 & 0 & 0 & 2 \\
\hline O. picata & 0 & 1 & 0 & 0 & 0 & 0 & 0 & - & - & 0 & - & 0 & - & 1 & 2 & 0 & 0 & 0 & 0 & 0 \\
\hline I. benmetit & 0 & 0 & 0 & 0 & 0 & 0 & 1 & 0 & 1 & 0 & - & 0 & 0 & 0 & 0 & 1 & 1 & 0 & 0 & 2 \\
\hline C. migrita & 0 & 0 & 1 & 0 & 2 & 0 & 1 & 0 & 1 & 0 & - & 1 & 0 & 0 & 1 & 1 & 0 & 1 & 0 & 2 \\
\hline C. rufescens & 0 & 1 & 0 & 1 & 3 & 0 & 1 & 0 & 1 & 0 & - & 1 & 0 & 0 & 1 & 1 & 0 & 0 & 0 & 2 \\
\hline 2. mufercens & 0 & 0 & 0 & 0 & 0 & 0 & 1 & 1 & 0 & 0 & - & 0 & 0 & 0 & 0 & 1 & 0 & 0 & 0 & 2 \\
\hline
\end{tabular}

\begin{tabular}{|l|c|c|c|c|c|c|c|c|c|c|c|c|c|c|c|c|c|c|c|}
\hline \multicolumn{1}{|c}{ Character } & 21 & 22 & 23 & 24 & 25 & 26 & 27 & 28 & 29 & 30 & 31 & 32 & 33 & 34 & 35 & 36 & 37 & 38 & 39 \\
\hline soforcula & 0 & 0 & 0 & 1 & 0 & 0 & 1 & 2 & 0 & 0 & 1 & 0 & 0 & 1 & 1 & 0 & 1 & $?$ & $?$ \\
\hline heterogaster & 0 & 0 & 0 & 1 & 0 & 1 & 0 & 0 & 0 & 1 & 0 & 0 & 0 & 1 & 1 & 0 & 1 & 1 & 0 \\
\hline umbrosa & 0 & 0 & 0 & 1 & 0 & 1 & 0 & 0 & 0 & 0 & 0 & 0 & 0 & 1 & 1 & 1 & 1 & 1 & 0 \\
\hline inquilina & 0 & 0 & 0 & 1 & 0 & 0 & 0 & 0 & 0 & 0 & 0 & 1 & 0 & 1 & 1 & 0 & 1 & $?$ & $?$ \\
\hline brevios & 0 & 0 & 0 & 1 & 0 & 1 & 0 & 0 & 0 & 0 & 0 & 0 & 0 & 1 & 1 & 1 & 1 & 1 & 0 \\
\hline fenusi & 0 & 1 & 0 & 1 & 0 & 0 & 1 & 0 & 0 & 0 & 0 & 0 & 0 & 1 & 1 & 1 & 1 & 1 & 0 \\
\hline bomnairi & 0 & 0 & 0 & 1 & 0 & 0 & 1 & 0 & 0 & 2 & 2 & 1 & 0 & 1 & 1 & 1 & 1 & 1 & 1 \\
cribripeninis & 0 & 1 & 0 & 1 & 0 & 0 & 1 & 0 & 0 & 2 & 2 & 1 & 0 & 1 & 1 & 1 & 1 & 1 & 1 \\
\hline sicuitera & 1 & 1 & 0 & 0 & 0 & 0 & 1 & 0 & 0 & 1 & 2 & 1 & 0 & 1 & 1 & 1 & 1 & 1 & 1 \\
\hline inermis & 1 & 1 & 0 & 0 & 0 & 0 & 1 & 0 & 0 & 1 & 2 & 1 & 0 & 1 & 1 & 1 & 1 & 1 & 1 \\
\hline caeca & 1 & 1 & 0 & 1 & 0 & 0 & 1 & 0 & 0 & 1 & 2 & 1 & 0 & 1 & 1 & 1 & 1 & $?$ & $?$ \\
\hline crassicomis & 0 & 1 & 0 & 0 & 0 & 0 & 1 & 0 & 0 & 1 & 2 & 1 & 0 & 1 & 1 & $?$ & 1 & $?$ & $?$ \\
\hline carinata & 0 & 1 & 0 & 0 & 1 & 0 & 1 & 0 & 0 & 1 & 2 & 1 & 0 & 1 & 1 & 1 & 1 & $?$ & $?$ \\
\hline forticomis & 0 & 1 & 0 & 2 & 1 & 0 & 1 & 1 & 1 & 1 & 2 & 1 & 0 & 1 & 1 & 1 & 1 & 1 & 0 \\
\hline
\end{tabular}


Tab. 1 (cont.): Character matrix. Explanation of abbreviations: $t=$ transitional $(0-1) ; ?=$ unknown; - = logically impossible; i = irrelevant.

\begin{tabular}{|c|c|c|c|c|c|c|c|c|c|c|c|c|c|c|c|c|c|c|c|}
\hline Character & 21 & 22 & 23 & 24 & 25 & 26 & 27 & 28 & 29 & 30 & 31 & 32 & 33 & 34 & 35 & 36 & 37 & 38 & 39 \\
\hline loebli & 1 & 0 & 0 & 1 & 0 & 2 & t & 1 & 0 & 1 & 2 & 1 & 0 & 1 & 1 & 0 & 1 & 1 & 0 \\
\hline seriepunctuta & 1 & 1 & 0 & 1 & 0 & 2 & 0 & 1 & 0 & 1 & 2 & 1 & 1 & 1 & 1 & 0 & 1 & $?$ & $?$ \\
\hline armata & 1 & 1. & 0 & 1 & 0 & 2 & 0 & 1 & 0 & 1 & 2 & 1 & 0 & 1 & 1 & 0 & 1 & 1 & 0 \\
\hline wrasei & 1 & 1 & 0 & 1 & 0 & 2 & 1 & 1 & 0 & 1 & 2 & 1 & 0 & 1 & 1 & 0 & 1 & 1 & 0 \\
\hline megalops & 0 & 0 & 0 & 1 & 0 & 2 & 1 & 1 & 0 & 1 & 2 & 2 & 0 & 1 & 1 & 0 & 1 & 1 & 0 \\
\hline formosana & 0 & 1 & 1 & 1 & 0 & 2 & 1 & 0 & 0 & 0 & 1 & 0 & 0 & 1 & 1 & 0 & 1 & 1 & 0 \\
\hline densepunctata & 0 & 1 & 0 & 0 & 0 & 2 & 1 & 0 & 0 & 1 & 1 & 2 & 0 & 1 & 1 & 1 & 1 & 1 & $?$ \\
\hline E. dectmana & 0 & 0 & 0 & 0 & 0 & 0 & 0 & 0 & 1 & 2 & 0 & 0 & 0 & 1 & 0 & 0 & 0 & 0 & 1 \\
\hline E. murina & 0 & 0 & 0 & 0 & 0 & 0 & $t$ & 0 & 0 & 2 & 0 & 0 & 0 & 1 & 0 & 0 & 0 & 0 & 1 \\
\hline O. picata & 0 & 0 & $t$ & 2 & 0 & 0 & 0 & 0 & 0 & 2 & 0 & 1 & 0 & 1 & 0 & 0 & 0 & 0 & 0 \\
\hline I. bernetti & 0 & 0 & 0 & 0 & 1 & 0 & $t$ & 1 & 0 & 1 & 0 & 0 & 0 & 1 & 0 & 0 & 0 & 0 & 0 \\
\hline C. nigria & 0 & 0 & 0 & 2 & 0 & 0 & 1 & 1 & 1 & 1 & 1 & 0 & 0 & 1 & 1 & 0 & 0 & 0 & 0 \\
\hline C.nescens & 0 & 0 & 0 & 2 & 0 & 0 & 0 & 1 & 1 & 1 & 0 & 0 & 0 & 1 & 1 & 0 & 0 & 0 & 0 \\
\hline 2. nffexcens & 0 & 0 & 0 & 0 & 0 & 0 & 0 & 0 & 0 & 2 & 0 & 0 & 0 & 0 & 0 & 0 & 0 & 0 & 0 \\
\hline
\end{tabular}

\begin{tabular}{|c|c|c|c|c|c|c|c|c|c|c|c|c|c|c|c|c|c|c|c|}
\hline Character & 40 & 41 & 42 & 43 & 44 & 45 & 46 & 47 & 48 & 49 & 50 & 51 & 52 & 53 & 54 & 55 & 56 & 57 & 58 \\
\hline sororcula & 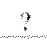 & $?$ & $\theta$ & $?$ & 0 & $?$ & 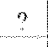 & $?$ & $?$ & . & $?$ & $?$ & $?$ & 1 & 1 & 1 & 0 & 0 & 2 \\
\hline heterogester & 0 & 0 & 0 & 1 & 0 & 2 & 1 & 1 & 1 & 1 & 1 & 0 & - & 1 & 1 & 1 & 0 & 0 & 2 \\
\hline umbrosa & 1 & 0 & 1 & 0 & 0 & 2 & 1 & 1 & 1 & 1 & 1 & 0 & 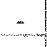 & 1 & 1 & 1 & 0 & 0 & 1 \\
\hline inqualina & $?$ & 2 & $?$ & $?$ & $?$ & $?$ & 3 & $?$ & $?$ & $?$ & $?$ & $?$ & $?$ & 1 & 1 & 1 & 0 & 0 & 3 \\
\hline brevios & 1 & 0 & 0 & 1 & 0 & 2 & 1 & 1 & 0 & 1 & 1 & 0 & - & 1 & 1 & 1 & 0 & 0 & 3 \\
\hline fenvesi & 0 & 0 & 0 & 0 & 0 & 2 & 1 & 0 & 0 & 1 & 1 & 0 & - & 1 & 1 & 1 & 0 & 0 & 3 \\
\hline bomairei & 0 & 0 & 0 & 1 & 0 & 0 & 2 & 1 & 0 & 1 & 1 & 0 & - & 1 & 1 & 1 & 0 & 0 & 1 \\
\hline cribripesnis & 9 & 0 & 0 & 1 & 0 & 0 & 2 & 1 & 0 & 1 & 1 & 0 & - & 1 & 1 & 1 & 0 & 0 & 1 \\
\hline siculifera & 0 & 0 & 1 & 0 & 1 & 0 & 1 & 1 & 0 & 1 & 1 & 0 & - & 1 & 1 & 1 & 0 & 0 & 1 \\
\hline inemis & 0 & 0 & 1 & 0 & 0 & 0 & 1 & 0 & 0 & 1 & 1 & 0 & - & 1 & 1 & 1 & 0 & 0 & 1 \\
\hline cacca & $?$ & $?$ & $?$ & $?$ & $?$ & $?$ & $?$ & $?$ & $?$ & $?$ & $?$ & $?$ & $?$ & 1 & 1 & 1 & 0 & 0 & 1 \\
\hline crassicom & $?$ & $?$ & $\eta$ & $?$ & 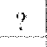 & $?$ & $?$ & $?$ & $?$ & $?$ & $?$ & $?$ & $?$ & $?$ & ? & $?$ & $?$ & $?$ & 1 \\
\hline carinata & $?$ & $?$ & $?$ & $?$ & $?$ & $?$ & $?$ & $?$ & $?$ & $?$ & $?$ & $?$ & $?$ & 1 & 1 & 1 & 0 & 0 & 1 \\
\hline fortionnis & 0 & 1 & 1 & 0 & 0 & 0 & 0 & 1 & 0 & 1 & 0 & - & - & 1 & 1 & 1 & 0 & 0 & 1 \\
\hline locbli & 2 & 1 & 1 & 0 & 1 & 0 & 1 & 1 & 0 & 1 & 1 & 1 & 0 & 1 & 1 & 1 & 0 & 0 & 2 \\
\hline serieptm & $?$ & 7 & $?$ & $?$ & $?$ & $?$ & $?$ & $?$ & $?$ & $?$ & $?$ & $?$ & $?$ & $?$ & $?$ & 3 & $?$ & $?$ & 2 \\
\hline armata & 2 & 1 & 1. & 0 & 1 & 0 & 1 & 1 & 0 & 1 & 1 & 1 & 1 & $?$ & $?$ & $?$ & $?$ & $?$ & 2 \\
\hline urasei & 2 & 1 & 1 & 0 & 0 & 0 & 1 & 1 & 0 & 1 & 1 & 1 & 0 & 1 & 1 & 1 & 0 & 0 & 2 \\
\hline megalops & 1 & 1 & 0 & 1 & 0 & 0 & 0 & 0 & 0 & 1 & 1 & 1 & 0 & 1 & 1 & 1 & 0 & 0 & 2 \\
\hline formosana & 0 & 1 & 0 & 1 & 0 & 1 & 1 & 0 & 0 & 11 & 1 & 0 & - & 1 & 1 & 1 & 0 & 0 & 2 \\
\hline densepunctata & $?$ & $?$ & $?$ & $?$ & $?$ & $?$ & $?$ & $?$ & $?$ & $z$ & $?$ & $?$ & $?$ & 1 & 1 & 1 & 1 & 1 & 2 \\
\hline E. decumana & 0 & 0 & 9 & 0 & 0 & 0 & 0 & 0 & 0 & 0 & - & - & - & 0 & 0 & 0 & 0 & 0 & $\mathrm{i}$ \\
\hline E. murina & 0 & 0 & 0 & 0 & 0 & 0 & 0 & 0 & 0 & 0 & - & - & - & 0 & 0 & 0 & 0 & 0 & $\mathrm{i}$ \\
\hline O. picata & 0 & 1 & 0 & 0 & 0 & 0 & 0 & 0 & 0 & 0 & - & - & - & 0 & 0 & 0 & 0 & 0 & $i$ \\
\hline 1. bennetti & 0 & 1 & 0 & 0 & 0 & 0 & 1 & 0 & 0 & 0 & - & - & - & 1 & 0 & 1 & 0 & 0 & $i$ \\
\hline C. nigrita & 0 & 1 & 0 & 0 & 0 & 0 & 0 & 0 & 0 & 0 & - & $\therefore$ & - & 0 & 0 & 0 & 0 & 0 & i \\
\hline C. rufescens & 0 & 1 & 0 & 0 & 0 & 0 & 0 & 0 & 0 & 0 & - & 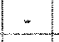 & - & 0 & 0 & 0 & 0 & 0 & $\mathrm{i}$ \\
\hline Z. mescens & 0 & 0 & 0 & 0 & 0 & 0 & 0 & 0 & 0 & 0 & - & - & - & 0 & 0 & 0 & 0 & 0 & i \\
\hline
\end{tabular}




\subsubsection{Conclusions}

For several reasons it is difficult to determine the intrageneric phylogenetic affiliations unequivocally. The main weakness of the character matrix (Tab. 1) is obviously the fact that the male sexual characters, which are of particular phylogenetic significance in Amarochara, of almost one third of the species are unknown. In addition, many external character are subject to considerable in-group and even intraspecific variation, so that it was sometimes difficult to determine the respective character state or to distinguish between homoplasies and secondary reductions. A transformation of the character matrix into a phylogenetic tree is here refrained from, not only because many of the basal ramifications and lineages are weakly supported, but also because this would require an examination of the species from other zoogeographic regions. Instead, some basic conclusions are here discussed in more detail.

Amarochara forms a monophyletic group constituted by numerous synapomorphies $(6$, $7,8,16,35,37,38,47,49,53,54,55)$. Within the genus, the best-defined monophylum is a species group from the Eastern Palaearctic region including $A$. loebli, $A$. armata, $A$. wrasei, $A$. megalops, and $A$. seriepunctata, which share several derived character states $(5,10$, 51), especially a posteriorly constricted head (character 4$)$. A. loebli and $A$. armata are apparently adelphotaxa, their monophyly constituted by character 44 . Together with $A$. seriepunctata and $A$. wrasei, they form the monophyletic sister group of $A$. megalops (characters $21,32,40,42,46,47)$. (The affiliations of $A$. seriepunctata are somewhat uncertain, as the male sexual characters of this species are unknown.) The monophylum $[($ loeblitarmata $)+$ wrasei+ seriepunctata $]+$ megalops is evidently the adelphotaxon of the Eastern Palaearctic monophylum formosana+densepunctata (see characters 12, 15, 26, 41 and $3,18,31$, respectively). The monophyly of formosana +densepunctata may actually be supported by more synapomorphies, but the male sexual characters of $A$. densepunctata are unknown.

The phylogenetic relationships within the remainder of Amarochara are somewhat more difficult to assess. It seems that $A$. umbrosa, the Nearctic species (A. inquilina, A. brevios, A. fenyesi), and some Himalayan species (A. sororcula, A. heterogaster) together form a monophyletic group, a hypothesis supported by characters $2,5,12,24,46$, and especially the derived condition of character 45. A closer relationship of $A$. umbrosa to the Nearctic species is suggested by character 5 . The monophyly of the remaining species $(A$. bonnairei, $A$. cribripennis, $A$. siculifera, $A$. inermis, $A$. caeca, $A$. crassicornis, $A$. carinata, $A$. forticornis) is only weakly supported (see characters $9,22,27,36$ ), because the derived condition is either not found in all of the species (thus implying secondary reductions) or not exclusive to this species group (and consequently implying convergent evolution in several species groups). There is little doubt that $A$. bonnairei and $A$. cribripennis, as well as A. inernis and $A$. siculifera are both monophyletic (see characters $30,43,46$, and characters 42,46 , respectively) and that they are adelphotaxa (cf. characters 1 and 39). However, their phylogenetic affiliations with $A$. caeca, $A$. crassicornis, and $A$. carinata are obscure, particularly because the male sexual characters of the latter species are unknown. This is also one of the reasons why the position of $A$. forticornis is doubtful. This species is characterized both by numerous plesiomorphies and by several autapomorphies. It may be the adelphotaxon either of all other Palaearctic and Nearctic Amarochara or of A. bonnairei and allied species. 


\subsection{The subgenera of Amarochara}

The species of Amarochara occurting in the Holarctic region have been attributed to three subgenera (see also section 1): Amarochara s. str., Lasiochara GANGLBAUER, and Mniobates MULSANT \& REY. They have been distinguished based on the following characters: absence/presence of an anterior impression on the abdominal tergum VI, the length of antennomere III, the presence/absence of a mesosternal carina, and the absence/presence of genal carinae (see e. g. GANGLBAUER, 1895).

If the existing subgeneric concept were applied, at least one or two additional subgenera would have to be erected to accommodate the species allied to $A$. loebli, as well as $A$. formosana and $A$. densepunctata (see section 3.3.1.2.). In addition, the subgenera would have to be redefined, especially to account for the species related to $A$. bonnairei. However, as outlined in the preceding chapter, the phylogenetic affiliations of several species (especially $A$. crassicornis, $A$. caeca, and $A$. carinata) and species groups are doubtful and, even more important, the species from other zoogeographic regions have not been reexamined. Therefore, the subgenera are here neglected (but not formally synonymized) and the species are attributed to five species groups: The umbrosa group ( $A$. umbrosa, A. sororcula, $A$. beterogaster, and the Nearctic species), the bonnairei group ( $A$. bonnairei, $A$. cribripennis, $A$. siculifera, $A$. inermis, $A$. caeca, $A$. splendens, and $A$. crassicornis), the forticornis group (A. forticornis and A. carinata), the loebli group (A. loebli, A. seriepunctata, A. armata, A. urasei, $A$. megalops), and the formosana group (A. formosana, $A$. densepunctata). The inclusion of $A$. carinata in the forticornis group is primarily based on the presence of a mesosternal carina and must be considered tentative until males of this species become available.

\subsection{Distribution and bionomics}

Species described in or transferred to Amarochara are known from the Holarctic, the Neotropical, the Oriental, and the Ethiopian region (see also section 1). However, apart from those occurring in the Holarctic region and some Ethiopian representatives, the species have not been reexamined.

Remarkably, Amarochara ecologically resembles the aleocharinae genera Callicerus GRAVENHORST (Athetini), Pseudosemiris MACHULKA (Athetini), and Iyobates KRAATZ (Oxypodini) in many ways, all of these taxa additionally sharing some morphological characters, such as a slender body and remarkably long and stout antennae (see ASSING, 1999, 2001). The collection data suggest that Amarochara species are univoltine and that reproduction and larval development take place in an unknown subterranean habitat in spring and early summer. Most, if not all the species are capable of flight, which explains why many species are apparently relatively widespread. Adult beetles have been collected from litter, moss, decaying organic matter, and other substrates in a wide variety of forested and open habitats. Amarochara larvae are unknown. For further details see the species sections. 


\section{The species of Amarochara}

\subsection{The ambrosa group}

The umbrosa group comprises six rather small species of Palaearctic (1 species), Eastern Palaearctic ( 2 species), and Nearctic distribution ( 3 species). They share the following derived characters: a more or less subrectangular head, usually with weakly marked posterior angles, partly to completely reduced genal carinae, a relatively short antennomere III, usually straight (i. e. not sinuate) posterior elytral margins, and an aedeagus with a moderately to very long apical part and a moderately long basal part of the internal tube, and with a usually reduced crista apicalis (see also section 3.3).

\subsubsection{Amarochara umbrosa (ERICHSON, 1837)}

Figs. 1-15, Maps 1-2

Calodera umbrosa ERICHSON, 1837: 304.

Amarochara umbrosa: THOMSON (1858).

Homalota tenuis HEER, 1839: MULSANT \& REY (1875).

Oxypoda funicularis HOCHHUTH, 1871; synonymy by GUSAROV (1992).

Amarochara (s. str.) umbrosa: GANGLBAUER (1895).

\section{Types examined}

Lectotype ${ }^{\star}$ [dissected and remounted], here designated: $5327 /$ umbrosa Er. Berol. Schüpp. / Lectotypus o Calodera umbrosa Erichson desig. V. Assing 2000 / Amarochara umbrosa (Erichson) det. V. Assing 2000 (MNHUB).

Paralectotypes: 2 o o , 1 sex? [abdomen missing]: Hist.-Coll. (Coleoptera) Nr. 5327 Calodera umbrosa Erichs. Berolin, Schüpp. Zool. Mus. Berlin / Paralectotypus Calodera umbrosa Erichson desig. V. Assing 2000 / Amarochara umbrosa (Erichson) det. V. Assing 2000 (MNHUB).

\section{Comments}

The original description of Calodera umbrosa is based on an unspecified number of syntypes collected near Berlin by Schüppel. In order to fix a single name-bearing type and to secure the present interpretation of the species, the male syntype is here designated as lectotype.

According to the respective curators in charge, the type(s) of Homalota tenuis HeER (type locality: "Geneva") were looked for, but not found, in the Heer collection in Zürich and in the collections of the BMNH, where, according to HORN et al. (1990), part of the types of the species described by HEER are kept (BRENDEIL, pers. comm.; MÜLLER, pers. comm.). A neotype designation is here refrained from, since the original description of $H$. tenuis leaves little doubt that it is conspecific with $C$. umbrosa ERICHSON. There is no clear evidence that the specimen collected by MÄRKEL in Genève, labelled as "tenuis Heer" and deposited in the collections of the MNHUB, is a syntype of Homalota tenuis. Its type status is here regarded as doubtful.

The synonymy of Oxypoda funicularis HOCHHUTH with Amarochara umbrosa was established by GUSAROV (1992) based on an examination of type material. 


\section{Additional material examined}

Great Britain: 1 o, Surrey, Dorking, Box Hill, in pile of cut grass, 21.VI.1988, leg. Owen (cOwe); 1 ex., Berkshire, Bradfield, leg. Joy (cOwe).

Sweden: Skåne: $10^{\star}$, Ignaberga, 14.IV.1961. leg. Istaelson (SMNO).

France: Bretagne: 1 ex., Rennes, leg. Oberthür (NMP). Pyrénées-Orientales: 1 ex., leg. Normand (MHNG). Poitou-Charentes: 1 ex., Vienne, Morthemer, leg. Masmin (NMP). Aquitaine: 1 ex., Dordogne, Cénac, St. Julien, cow dung, VII.1974, leg. Kanaar (UKNHM). Centre: 1 ex., Vendôme (MHNG). Nord-Pas-de-Calais: 1 ex., Lille (DEI). Picardie: 2 exx., Laigneville, leg. Méquignon (MHNG). Île-deFrance: 1 ex., Paris (DEI); 1 ex., Paris ["Lutet."], leg. Fairmaire (DIE). Midi-Pyrénées: *1 9 , Ignaux, 25.VII.1970, leg, Tronquet (cTro). Rhône-Alpes: 1 ex., Ain, Ctozet, 1200m, VII.1961, leg. Toumayeff (MHNG); 1 ex., Écully, leg. Andras (MHNG); 1 ex., Lyon (NHMB); 3 exx., "Rhone" (MNHUB, NHMB). Provence: 19 , Alpes-de-Haute-Provence, Col de la Cayolle, 1600m, VI.1988, leg. Wunderle (cAss).

Germany: Schleswig-Holstein: 1 ex., Lübeck, 1914, leg. Benick (MHNG); 1 ex., Lübeck, Bahnhof, 8.VIII.1917 (DEI). Niedersachsen: 1 ex., Goslar, leg. Jacobs (cAss). Nordrhein-Westfalen: 1 ex., Aachen, V.33, leg. Rosskothen (SMTD); 2 exx., Düsseldorf, Meererbusch, 4.V.1938, leg. Ermisch (SMTD); 1 ex., same data, but 10.VI.1935 (MNHUB); 1 ex., Düsseldorf, leg. Hagens (DEI); 1 ex., Porta Westfalica, Barkhausen, IV.1958 (NMB); 1 ex., Barkhausen, 20.IV.1950 (NMB); 1 ex., Stemweder Berge (SE Dümmer), 25.IV.1954, leg. Kerstens (SMNO); 1 \% , Troisdorf, Wahner Heide, car-net, IV.1987, leg. Wunderle (cWun); 1 ex., Barmen (DEI); 1 ex., Bonn (DEI); 4 exx., Krefeld, leg. Czwalina (DEI). Saarland: 4 exx., Saarlouis (FIS). Rheinland-Pflalz: 19, Sarburg/Saar, Taben-Rodt, car-net, 6.VI.1996, leg. Renner (cRen); $2 \sigma^{\circ}$, Nahetal, Heinzenberg, 17.V.1986, leg. Wunderle (cWun); $10^{*}$, Staudernheim, Nahe-Auen, 5.V.1987, leg. Wunderle (cWun); 2 exx., Schloßböckelheim, 25.VI.1987, leg. Wunderle (cWun); 2 exx., Kastellaun, V.1965, leg. Lohse (MHNG); 3 exx., Boppard, 2.\&16.V.1937, leg. Ermisch (SMTD); 1 ex., Bacharach, Steeg, Weinberge, 20.V.1996, leg. Köhler (cKöh); 2 exx., Nahetal, Boos, sifted on xerothermous slope, 20.\&25.V.1988, leg. Wunderle (cWun, cAss); 4 exx., Nahetal, Martinstein, xerothermous slope, 8.V.1988, leg. Wunderle (cWun); 1 ex., Grünstadt, leg. Fleischer (NMP); 1 ex., Deidesheim, leg. Eppelsheim (DEI); 2 exx., Dürkheirn (NHMN); 1 ex., Heidesheim (NHMN); 1 ex., "Ahr" (DEI). Baden-Württemberg: 19 exx., Wimpfen, leg; Scriba (FIS); 1 ex., Markgtöningen, 23.III.1955, leg. Köstlin (SMNS); 1 o , Nimburg, 27.V.1980, leg. Lunau (SMNS); 4 exx., Heilbronn, 29. IV.1918 (MNHUB). Hessen: 1 ex., Frankfurt (DEI); 3 exx., Darmstadt, 13.VII.1957, leg. Vogt (FIS); 1 o, 1 ex., Dautphe-Buchenau, Katzenbachtal, car-net, 26.IV.1987, leg. Wunderle (cKöh, cAss); $1 \sigma^{*}$, same data, but 9.V.1987 (cWun); 1 ex., Wattenheim (FIS); 2 exx., Ober Lais, leg. Scriba (FIS); 1 ex., Dornholzhausen, 7.VII.1895, leg. Sattler (FIS); 1 ex., Schwanheim, 18.VI.1905, leg. Sattler (FIS); 1 ex., "Rheinhessen" (FIS); 2 exx., locality not specified (DEI). Bayern: 1 ex., Seligenstadt, leg. Scriba (FIS); 1 ex., Bad Kissingen, 1917 (FIS); 2 exx., Spessart, 12.X.1959, leg. Vogt (FIS); 2 exx., München (NHMW); 1 ex., Niederbayern, Birnbach, 24.V.1907 (NHMB). Mecklenburg-Vorpommern: 1 ex., "Mecklenburg" (NHMW). Sachsen-Anhalt: 4 exx., Thale/Harz, Waldkater-Fenster, 26.VI.1953, leg. Dorn (SMTD, cAss); 4 exx., Thale/Harz, 25.V.1941 (MNHUB); 1 ex., Thale (MNHUB); 2 exx., Harz, Brocken, 8.VI.1922, leg. Linke (SMTD); 2 exx., Harz, "Brockenscheitel", 12.VII.1913 \& 14.VI.1914, leg. Petry (MNHUB); 3 exx., Harz, Bodetal, 25.V.1941 (MNHUB); 1 ex., Bodetal, 31.V.1952, leg. Dorn (cAss); 1 ex., Quedlinburg (MNHUB); 1 ex., Schönburg near Naumburg, 2.V.1930, leg. Maertens (MNHUB); 1 ex., Leißling, 29.IV.1928 (MNHUB); 1 ex., Magdeburg (SMTD). Berlin/Brandenburg: 2 exx., Berlin (DEI, MNHUB); 1 ex., Berlin, IV.1890 (NHMW); 1 ex., Fürstenberg, leg. Konow (MNHUB); 7 exx., Frankfurt/ Oder, leg. Schukatschek (FIS, MNHUB); 2 exx., Kalkberge near Rüdersdorf, leg. Neresheimer (DEI). Thüringen: 1 ex., Schwaiza near Suhl, 1.V.1989, leg. Sieber (cVog); 11 exx., Weißenfels, Schkortleben, 28.IV.1929, leg. Dorn (MNHUB, SMTD); 1 ex., Dornburg, 15.VIII.1909 (SMTD); 2 exx., Bad Frankenhausen, 17.IV.1919, leg. Petry (MNHUB); 1 ex., same data, but 8.IV.1920 (MNHUB); 1 ex., Frankenhausen, NSG Kattenburg, 14.V.1976, leg. Zerche (DEI); 1 ex., Kyffhäuser, 9.IV.1916, leg. Petry (MNHUB); 10 , NSG "Apfelstädter Ried", 15.V.1985, leg. Weipert (NME); 1 ex., Spitzberg, 27.VII.1952 (SMNS); 1 ex., locality not specified, VIII.1929 (SMTD). Sachsen: 1 ex., Oberlausitz, Rosenthal, 11.VII.1983, leg. Vogel (cRen); 1 ex., Herrnhut near Löbau, 25.VI.1977, leg. Sieber (cVog); 1 ex., Kamenz, Rosenthal, 11.VII.1983, leg. Vogel (cVog); 1 ex., Neschwitz, 11.VII.1983, leg. Vogel (cVog); 1 ex., Königswartha, 11.VII.1983, leg. Vogel (cWun); 1 ex., Leipzig, Zöbigker, 1.VIII.1942, leg. Linke (cVog); 1 ex., same data, but 14.VIII.1933 (SMTD); 4 exx., same data, but 19.\&22.VII.1943 (SMTD); 1 ex., same data, but 14.VIII.1934 (SMTD); 2 exx., same data, but 12.VII.1934 (SMTD); 1 ex., same data, but 18.VIII.1936 (cAss); 1 ex., same data, but 29.VI.1909 (SMTD); 6 exx., same data, but 6.VIII.1909 (SMTD); 2 exx., same data, but 28.\&30.VII.1908 (SMTD); 3 exx., Leipzig, Lindenthal, 19.IV.1914, leg. Linke (SMTD); 1 ex., Leipzig, Donna, 5.VII.1903 (SMTD); 1 ex., Leipzig, Dölzig, 12.II.1911, leg. Linke (SMTD); 1 ex., Leipzig, Widrow B., 21.V.1933, leg. 
Linke (SMTD); 1 ex., Leipzig, Altenhain B., 19.V.1929 (SMTD); 1 ex., S-Leipzig, 20.IV.1966, leg. Ermisch (SMTD); 3 exx., Leipzig, Grossteinberg, 26.V.1929, leg. Linke (SMTD); 1 ex., Bahren, 1.V.1904 (SMTD); 2 exx., Leipzig, exact locality illegible, 18.VI.\&24.X.1908, leg. Linke (SMTD); 1 ex., Leipzig, exact locality illegible, 11.VII.1908, leg. Linke (SMTD); 1 ex., Leipzig, Nonnenholz, 13.VI.1943, leg. Dorn (MNHUB); 2 exx., Brandis, 20.VII.1913, leg. Linke (SMTD); 1 ex., Wechselburg, 5.VII.1977, leg. Zerche (DEI); 1 ex., Guttau, car-net, 24.V.1985, leg. Schülke \& Heinig (MNHUB); 2 exx., Osterzgebirge, Müglitz, 22.VI.1954, leg. Linke (SMTD); 1 ex., Stollberg, 13.VIII.1921, leg. Uhmann (DEI); 4 exx., Zwrickau, Brückenberg, 23.\& 27.IV.1943, leg. Dorn (MNHUB); 1 ex., locality not specified (NHMW).

Switzerland: Genève: 1 ex., Les Baillets, cat carrion, VIII.1985, leg. Besuchet (MHNG); 1 ex., London, 1954, leg. Toumayeff (MHNG); 1 ex.: 5366 / Hist.-Coll. (Coleoptera) Nr. 5366 Homalota tenuis Heer Geneva, Märk. Zool. Mus. Berlin / tenuis Heer Genev. Märk. (MNHUB). Locality not specified: 2 exx. (DEI, NHMW).

Austria: Vorarlberg: 1 ex., Bregenz (NHMW). Tirol: 1 ex., Hall, leg. Ammann (TLMFI); 1 ex., Inntal, leg. Ammann (TLMFI); 1 ex., Innsbruck, Mühlau, Scheibenbühel, 25.V.1944, leg. Wörndle (TLMFI); 1 ex., Schwaz, fungus, 2.V.1947, leg. Kofler (TLMFI); 1 ex., same data, but 14.VII.1948 (TLFMI); 1 ex., Schwaz, Tratzberg, 27.III.1948, leg. Kofler (TLMFI); 1 ex., Schwaz, Arzberg, 29.VI.1950, leg. Kofler (TLMFT); 2 exx., Schwaz, Paulinum, compost, 7.VI.1956, leg. Kofler (TLMFI). Oberösterreich: 4 exx., Windischgarsten, leg. Skalitzky (FIS, NHMW). Niederösterreich/Wien: 4 exx., Wien (FIS, NHMW); 1 ex., Bad Deutsch Altenburg, leg: Moczarski (NHMW); 3 exx., Lobau near Wien, leg. Scheerpeltz (NHMW); 1 ex., Oberwalden near Wien, leg. Winkler (NHMW); 1 ex., Wien, Strebersdorf, IV.-V.1941, leg. Moczarski (NHMW); 9 exx., Mödling, 14.V.1966 (NMP); 1 ex., Wallmersdorf, leg. Scheerpeltz (NHMW); 1 ex., Felixdorf, 15.VIII.1965, leg. Gotz (SMNS); 2 exx., Vöslau (NHMW); 1 ex., Lainzer Tiergarten, leg. Scheerpeltz (NHMW); 1 ex., Oberweiden (NHMW); 3 exx., Wiener Wald, leg. Skalitzky (NHMW, SMNS, SMTD). Steiermark: 11 exx., Turnau (NNHUB, NHMW); 1 ex., Graz, leg. Strupi (NHMW). Kärnten: 1 ex., Wolfsberg, 8.VII.1909, leg. Schaaff (FIS); 7 exx., Eisenkappel, 15,-29.VII.1923, leg. Scheerpeltz (NHMW); 1 ex., Eisenkappel, VII.-VIII.1934, leg. Scheerpeltz (NHMW); 1 ex., Eisenkappel (SMNS); 3 exx., Navernig near Eisenkappel (NHMB, SMNS). Locality not specified: 1 ex., "Austria" (FIS).

Italy: Trentino-Alto Adige: 2 exx., Bressanone, 8.V.1957, leg. Peez (TLMFI, cAss); 3 exx., Bressanone, garden, 3.VI.1956, leg. Peez (TLMFI); 2 exx., same data, but 20.IV.1957 (TLMFI); 1 ex., same data, but 30.IV.1957 (TLMFI); 1 ex., Bressanone, Tochötscher Heide, 29.VII.1956, leg. Peez (TLMFI); 1 ex., Bolzano, "Moritzing" (DEI); 1 ex., Bolzano, Siusi, leg. Schuster (NHMW). Piemonte: 1 ex., locality not specified, leg. Baudi (DEI). Lombardia: 1 ex., Dalmine, 23.IV.92 (MHNG). Veneto: $20^{\circ} \sigma^{\circ}, 2 q$ q, 1 ex., Grezzana, car-net, 14.\&17.VI.1989, leg. Renner, Wunderle (cRen, cWun, cAss); 1 ex., alta Val d'Tllasi (VR), carnet, 19.V.1979, leg. Zanetti (cZan); 1 ex., Valeggio (VR), car-net, 8.VI.1979, leg. Zanetti (cZan); 2 exx., between Bussolengo (VR) and Sommacampagna (VR), car-net, 24.VI.1969, leg. Zanetti (cZan); 1 ex., between Vestenavecchia and Tregnago, car-net, 20.IV.1996, leg. Zanetti (cZan). Friuli-Venezia Giulia: 3 exx., Trieste, Basovizza, leg. Moczarski \& Scheerpeltz (NHMWW); 1 ex., Istria, Mt. Maggiore, 2.-8.VI.1931, leg. Stöcklein (NHMB). Toscana: 1 ex., Arezzo, 1942, leg. Gagliardi (SMTD). Sicilia: $2 \sigma^{\pi} \sigma^{\star}$, Noto Antica, carnet, 16./17.VII.1991, leg. Zanetti (cZan, cAss); 1 ex., Messina, 5.III.1942 (cAss).

Poland: 1 ex., Drewnica, 24.V.1919 (UKNHM); 2 exx., Silesia, Legnica ["Liegnitz"], leg. Gerhard (DEI, NMP); 12 exx., Legnica (NHMW); 1 ex., Silesia, "Wigstein"; 3 exx., "Schlesien", leg. Letzner (DEI); 1 ex., Wroclaw ["Breslau"], 8.V.1888 (FIS); 1 ex., ca. $10 \mathrm{~km}$ SE Wrocław, V.1902 (FIS); 3 exx., Niemcza ["Nimptsch"], VII.1902 (FIS); 1 ex., Niemcza, VII.1905 (FIS). Locality not specified: 2 exx. (NHMW).

Polish or Czech territory: 6 exx., Silesia, Cieszyn ["Teschen"], leg. Wanka (DEI, MNHUB, NHMB, NHMW); 1 ex., "Schlesien" (NHMB).

Czech Republic: Bohemia: 1 ex., Zbečno, 7.VI.1908, leg. Roubal (MHNG); 7 exx., Brandýs n.L., leg. Skalitzky (NHMW); 56 exx., Praha, leg. Lokay, Skalitzky (NHMTW, SMNS, SMTD, cAss); 1 ex., S Praha, Vrané nad Vltavou, leg. Krasa (NMP); 1 ex., Praha, Motol, leg. Machulka (NMP); 1 ex., Praha, Chuchle, 11.VI.1920 (NMP); 6 exx., S Praha, Davle, leg. Machulka (NMP); 1 ex., same locality, 8.VII.1917 (NMP); 1 ex., same locality (NMP); 8 exx., S Praha, Modéany, 1.VIII.1918 (NMP); 5 exx., N Praha, Roztoky, 5.VI.1910 (NMP); 1 ex., Okoli Prahy, 20.VI.1912 (NMP); 1 ex., Krkonoše, 1919, leg. Obenberger (NMP); 1 ex., Týn nad Vltavou (NMP); 8 exx., Praha, Śárka, leg. Klička (NMP); 3 exx., Praha, Śárka, leg. Machulka (NMP); 1 ex., Kolín, Velký Osek, 1.VI.1902 (NMP); 1 ex., Castolovice, leg. Roubal (MHNG); 4 exx., locality not specified or illegible (DEI, NHMB, NHMW, NMP). Moravia: 1 ex, Ostrava, leg. Zoufal (NHMW); 16 exx., Prostějov ["Prossnitz"], leg. Zoufal (FIS, MHNG, NHMW, NMP); 3 exx., Brno, leg. Fleischer (NMP); 1 ex., Adamov, leg. Fleischer (NMP); 1 ex., Kolovraty (NMP); 2 exx., Prokopov (NMP); 1 ex., Mikulov, 20.V.1966, leg. Lohse (MHNG); 1 ex., Čejč , leg. Roubal (DEI); 2 exx., locality not specified (NMP). 
Slovakia: 23 exx., Vysočany, 6.VIII.1919 (NMP, cAss); 11 exx., same locality, 12.VIII.1918 (NMP); 9 exx., same locality, 30.VII.1919 (NMP); 19 exx., same locality, 9.VII.1919 (NMP); 1 ex., Zliechov, 25.IV.1917 (NMP); 4 exx., Košice, 1926, 1928, 1929, leg. Machulka (NMP); 1 ex., Murán̆, leg. Machulka (NMP); 1 ex., Zvolen, leg. Roubal (MHNG); 2 exx., Hrhov, 3.\&7.V.1967, leg. Lohse (MHNG); 1 ex., Banská Bystrica, V.1934, leg. Roubal (MHNG); 1 ex., Bratislava, leg. Weber (NHMW).

Slovenia: 6 exx., Bled, 17.VIII.1912 (NMP, cAss); 8 exx., 8.VIII.1912 (NMP); 1 ex., Maribor, leg. Lang (NHMW); 2 exx., "Carniolia" (DEI):

Hungary: 1, Balatonfüred, 27.V.1999, leg. Renner (cRen); 1 ㅇ, Bugac National Park, grassland, pitfall, 19.V.1982, leg. Galle (cAss); 1 ex., Budapest (FIS); 1 ex., Pécs, leg. Kaufmann (NHMW).

Romania: 1 ex., Bihor, leg. Fleischer (NMP); 1 ex., Azuga, "Rotenturm-Pass", leg. Breit (NHMW); 1 ex., Băile Herculane ["Herkulesbad"], leg: Winkler (SMNS).

Croatia: 1 ex., Mala Capella near Čanak, 700m, 2.V.1990, leg. Wunderle (cWun); 1 ex., between Plitvice and Josipdol, car-net, 9.V.1990, leg. Wunderle (cAss).

Bosnia-Herzegovina: $30^{\star}, 3 \%$ \% 10 exx., Gromiljak, 6.V.1990, leg. Wunderle (cWun, cAss); $20^{\star} 0^{*}$, Vlasic Planina, 600-1700 m, car-net, 5.\&6.V.1990, leg. Wunderle (cWun, cAss); 2 exx., Kraljevska-Sutjeska, 5.V.1990, leg. Wunderle (cWun); 1 ex., Bjelašnica planina, leg. Leonhard (DEI); 1 ex., "Herzegowina", leg. Wanka (FIS); 1 ex., "Central-Bosnien", leg. Reuter (FIS); 1 ex., SWW Sarajevo, Pazaric, 4347N, $18^{\circ} 08 \mathrm{E}, 18 . \mathrm{VI} .1936$, leg. Linke (SMTD); 1 ex., W Sarajevo, Ilidua, X.1936, leg. Winneguth (SMTD); 1 ex., Trebinje (SMTD).

Yugoslavia: 3 ex., Serbia, Rusna, leg. Hensch (FIS).

Russian Federation: 1 ex., Orel [=Orjol], Melyn, 18.VT.1943, leg. Linke (SMTD); 1 ex., same data, but 25.V.1943 (cAss); 1 ex., Altai, Batnaul, 31.V.1919, leg. Babiy (NHMW); 10 , Novosibirsk, Akademgorodok, 23.-31.V.1986, leg. Schawaller (cAss).

China: $20^{\star} \sigma^{*}, 2$ o 9 [1 9 with mature egg in ovaries], Hebei, Yongnian, $36^{\circ} 47 \mathrm{~N}, 114^{\circ} 30 \mathrm{E}, \mathrm{D}-\mathrm{Vac} \&$ Barber traps, VI-XI.1995, leg. Shuiqiang Li (cAss, cSch).

Locality not specified, not identified, ambiguous, or illegible: 4 exx., "Cibulka, Zeman" (NMP); 5 exx., "Cibulka" (NMP); 2 exx., Eubna, leg. Klička (NMP); 1 ex., Sáriš (NMP); 1 ex., "Hungaria" (MNHUB); 1 ex., "Europa", leg. Scriba (FIS); 4 exx., "Ottwitz", leg. Letzner (DEI); 6 exx., "Lindb" [?] (FIS); 1 ex., "Hung. occ." (NHMW); 29 exx. (DEI, FIS, NHMW).

\section{Redescription}

2.2 - $3.2 \mathrm{~mm}$ (abdomen fully extended). Colour of body dark brown to blackish, often with the elytra lighter, yellowish brown to brown, and the posterior margins of the abdominal segments dark ferrugineous; antenna dark brown to blackish brown, legs light brown to brown. Integument shining; forebody usually without appreciable microsculpture (sometimes traces of microsculpture may be present on head and pronotum); abdomen at most with barely noticeable microsculpture.

Head of subquadrate shape, approximately as wide (across eyes) as long; eyes very large and prominent, distinctly projecting from lateral outline of head and only slightly shorter than postgenae in dorsal view, the latter almost parallel for about half their length. Puncturation very fine and sparse. Genal carina reduced. Antenna as in Fig. 5, antennomere I with pronounced dorso-apical furrow. Mouthparts as in Figs. 1-4.

Pronotum approximately as wide as long and 1.2 - 1.3 times as wide as head; maximal width usually approximately halfway between anterior angles and middle; puncturation highly variable, very fine to distinct, usually denser and more distinct than that of head. Elytra at suture $0.90-0.95$ times the length of pronotum and approximately $1.20-1.25$ times as wide as pronotum; posterior margin near posterior angles not or only indistinctly sinuate; puncturation weakly granulose and somewhat denser than that of pronotum. Hind wings fully developed. First metatarsomere approximately as long as the combined length of the two following tarsomeres. Mesosternum with microsculpture 
composed of meshes of various lengths, without median carina; mesosternal process apically \pm narrowly truncate or rounded.

Abdominal terga III-V with moderately deep, tergum VI without appreciable anterior impression; sterna III-V anteriorly constricted; puncturation fine, but distinct, moderately dense (though the density is subject to some intraspecific variation), and more or less evenly distributed on dorsal surface; tergum $\mathrm{X}$ as in Fig. 6.

$\sigma$ : posterior margin of tergum VIII slightly convex, with a row of moderately long and a row of long thin marginal setae (Fig. 11); posterior margin of sternum VIII distinctly convex, with two rows of long thin setae (Fig. 12); aedeagus with ventral process of median lobe in ventral view relatively broad, in lateral view weakly curved, and with basal fold; crista apicalis and crista proximalis reduced; internal sac with very long flagellum (Figs. 8, 9); apical lobe of paramere relatively short and broad (Fig. 7).

$\%$ : posterior margin of tergum VIII of similar chaetotaxy as in $\sigma^{\pi}$, but slightly more convex (Fig. 13); posterior margin of sternum VIII weakly convex, row of marginal setae very weakly modified, relatively thin, and shorter than in $\sigma^{*}$ (Fig. 14); spermatheca as in Fig. 10.

\section{Comparative notes}

From other Western Palaearctic congeners, $A$. umbrosa is distinguished by the combination of dark colour, small body size, more slender antennae, an almost subrectangular head shape, large eyes, the absence of genal carinae, and especially by the morphology of the aedeagus. For separation from similar species occurring in the Nearctic and the Eastern Palaearctic region see the comparative notes below those species and the key in section 5 . In the collections examined, $A$. umbrosa was most frequently confused with Calodera aetbiops (GRAVENHORST), with which it shares a similar general appearance (size, proportions, slender pronotum) and the massive antennae, from which it is, however, distinguished by the much sparser puncturation and more distinct shine of forebody and abdomen, the different shape of antennomere I, and by the completely different morphology of the sexual characters.

\section{Distribution and bionomics}

A. umbrosa is widespread in the southern and central parts of the Palaearctic region from Spain in the West to China in the east (Maps 1, 2). In the north and northwest it reaches the south of Norway, Sweden, and Finland, almost all of Denmark, and southern England (see also Fowler (1988), HANSEN (1964), HANSEN (1996), Horion (1967), JOY (1932), LUNDBERG (1995), and SILFVERBERG (1992)). In the west it is known from vatious regions in France (material examined; HORION, 1967). There is only a single record from Spain (north of Madrid) (GAMARRA, 1987), which, however, requires verification and is not included in Map 1. It occurs in all of Italy from the Alps in the north to Sicily in the south (LUIGIONI, 1929, WÖRNDLE, 1950), ZANETTI, 1995, and material examined). It is known from some localities in Switzerland and all Austrian regions (HORION, 1967, HugENTOBLER, 1966, SCHEERPELTZ, 1968, and material examined). In Germany, the species is widespread, but has not been reported from Niederelbe (HORION, 1967, KÖHLER \& KLAUSNITZER, 1998, and material examined); it is here for the first time reported from Mecklenburg-Vorpommern and Saarland. It is known from 


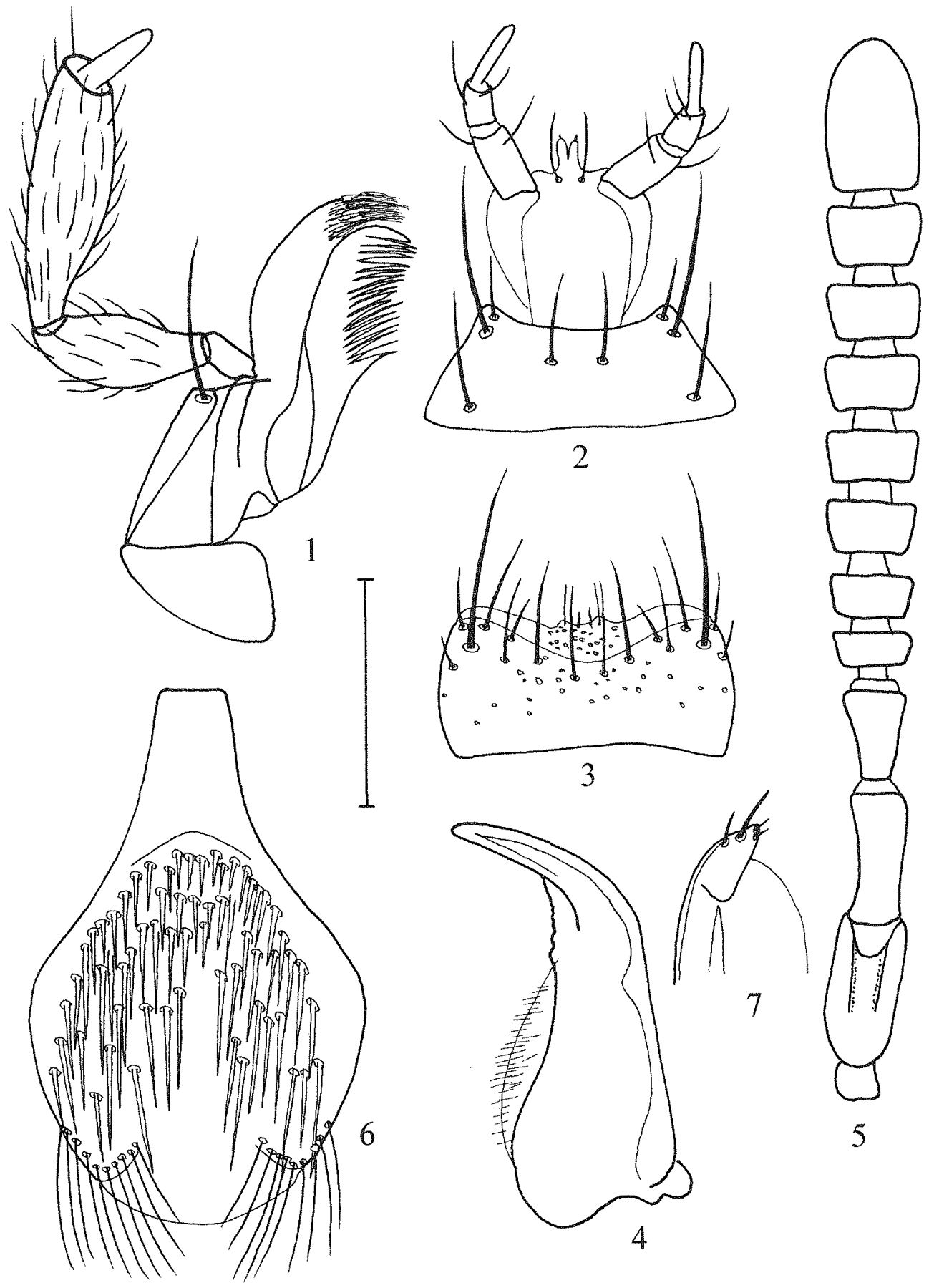

Figs. 1-7: Amarochara umbrosa (ERICHSON). Maxilla (1); labium (2); labrum (3); right mandible (4); antenna, pubescence omitted (5); tergum X (6); apical lobe of paramere (7). Scale: 1-4, 6-7:0.1 mm; $5: 0.2 \mathrm{~mm}$. 


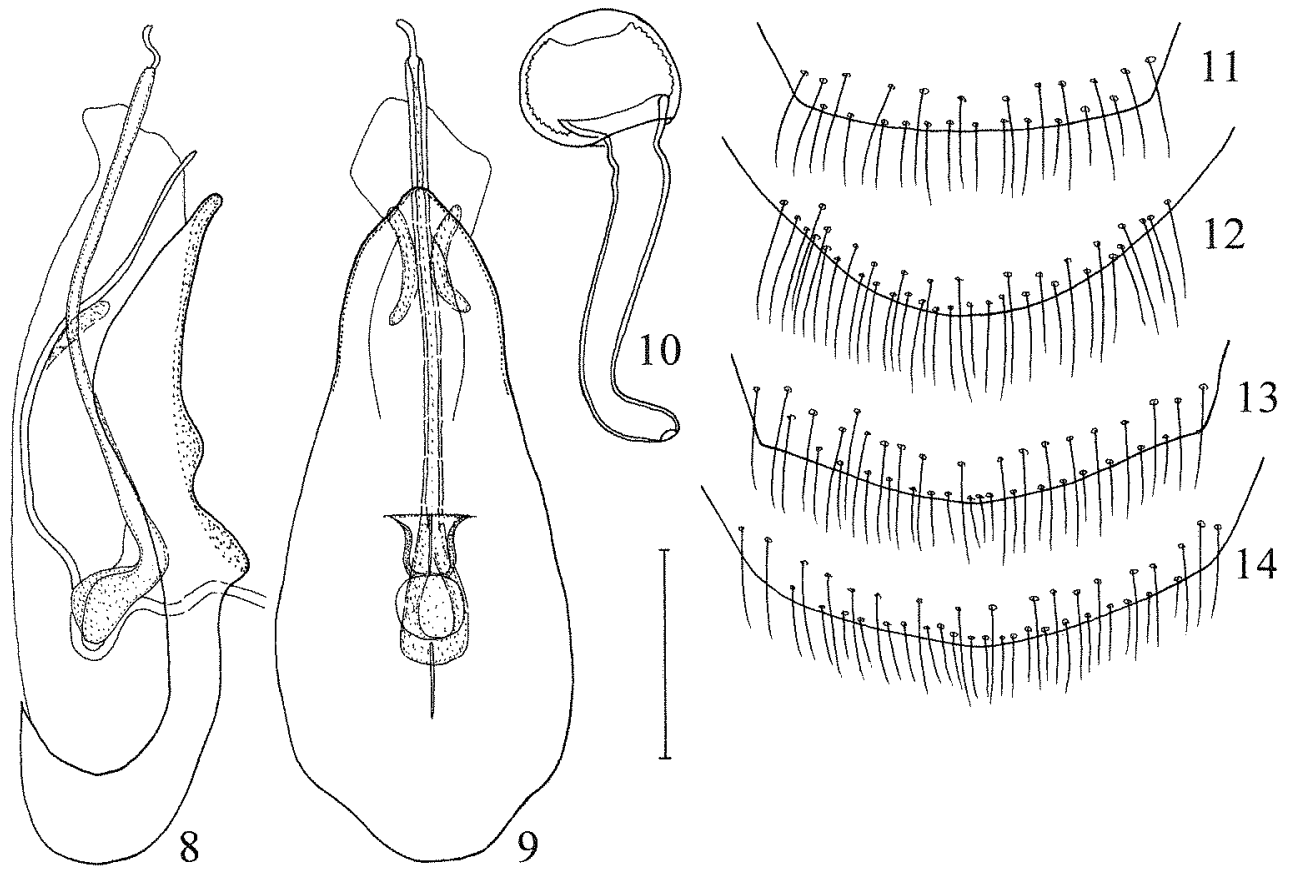

Figs. 8-14: Amarochara umbrosa (ERICHSON). Median lobe of aedeagus in lateral and in ventral view $(8,9)$; spermatheca (10); posterior margin of of tergum VIII (11); posterior margin of of sternum VIII (12); posterior margin of $q$ tergum VIII (13); posterior margin of $q$ sternum VIII (14). Scale: $0.1 \mathrm{~mm}$.

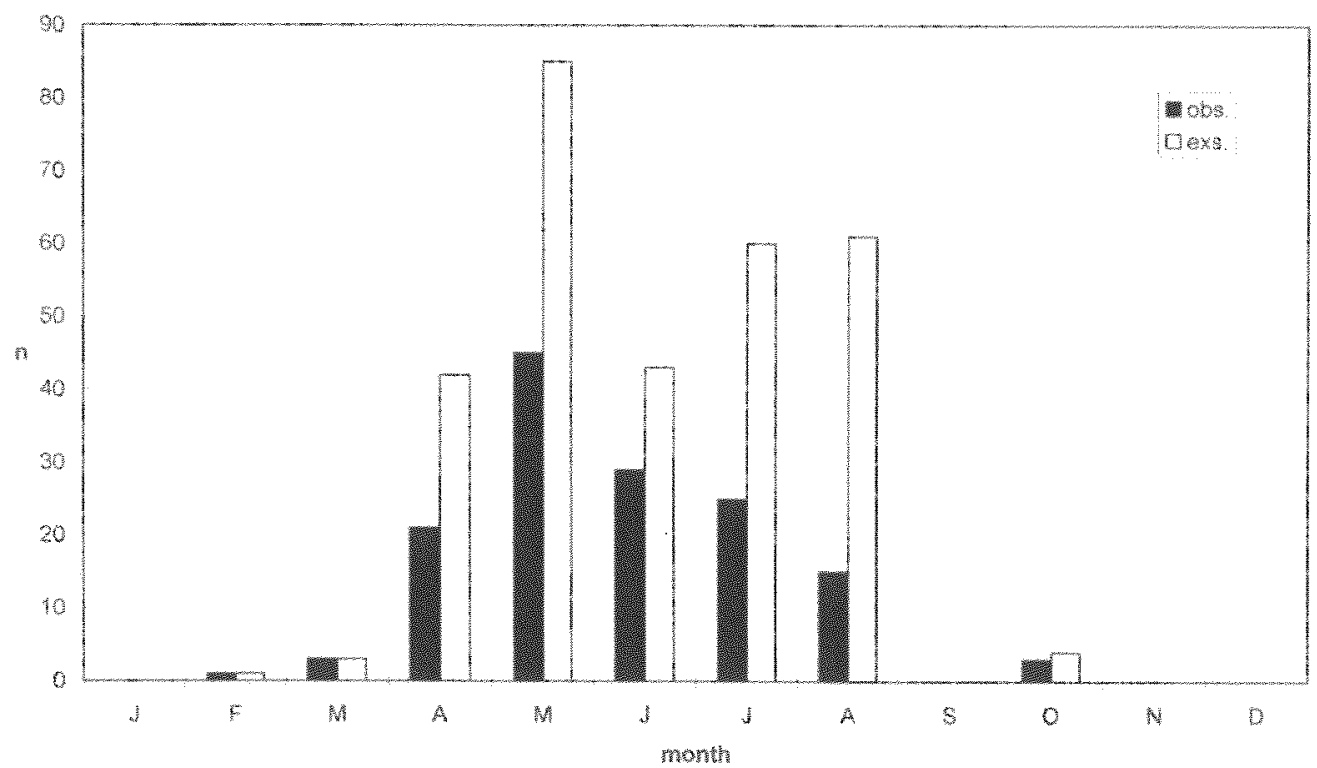

Fig. 15: Amarochara umbrosa (ERICHSON). Seasonal phenology based on material examined. Abbreviations: obs. $=$ number of observations or samples; exs. $=$ number of specimens. 
Beitr. Ent. 52 (2002) 1

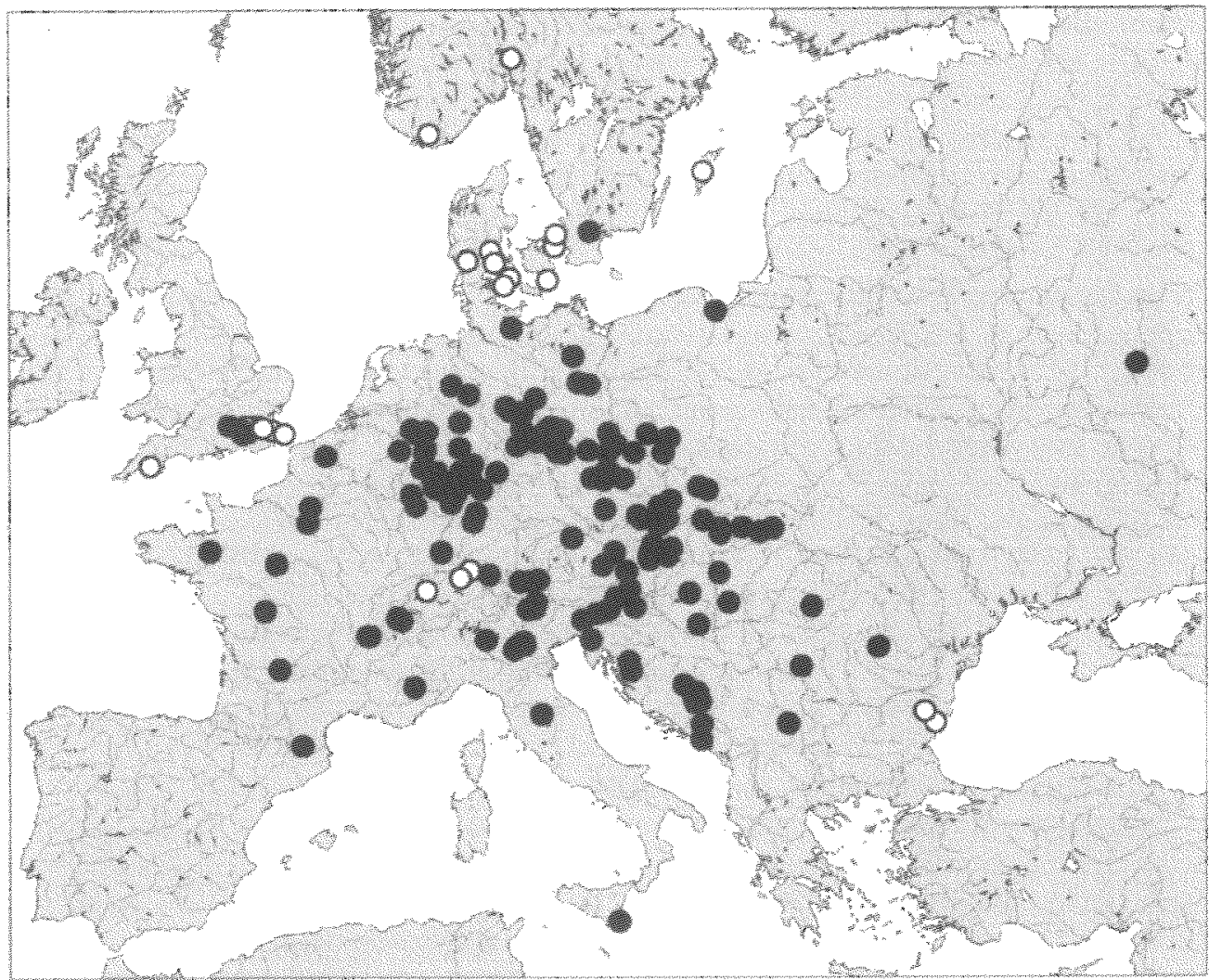

Map 1: Western Palaearctic distribution of Amarochara umbrosa (ERICHSON) based on revised records (filled circles) and selected literature records (open circles).

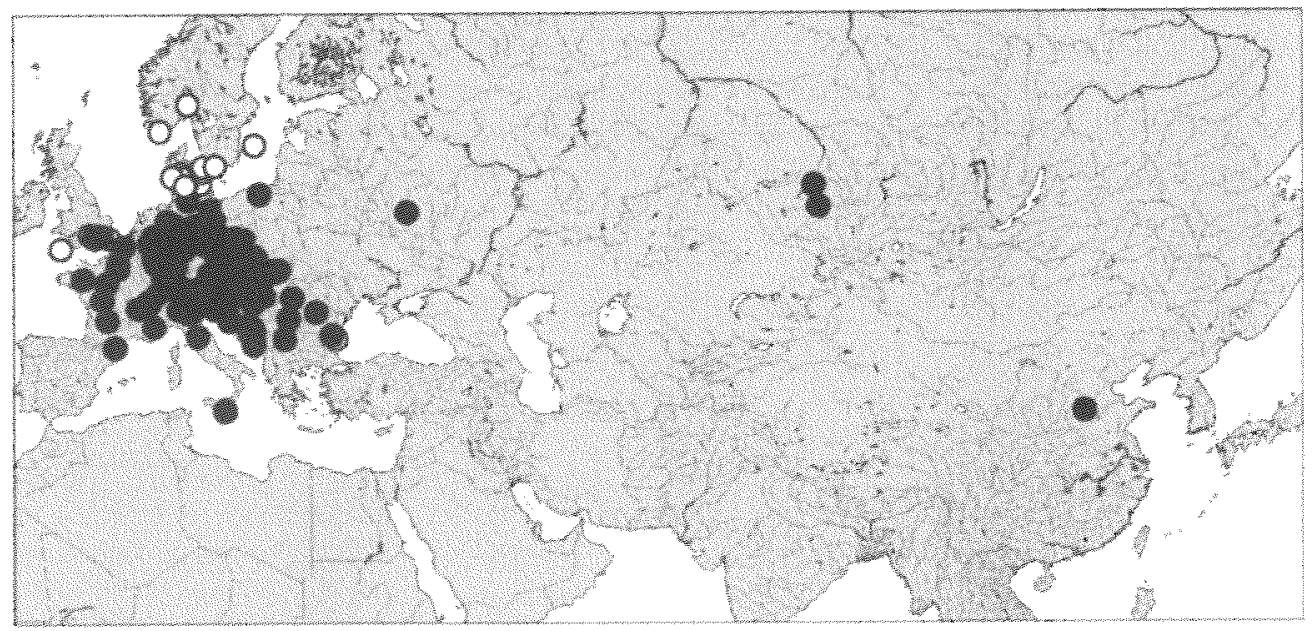

Map 2: Palaearctic distribution of Amarochara umbrosa (ERICHSON) based on revised records (filled circles) and selected literature records (open circles). 
Poland, the Czech Republic, Slovakia, Slovenia, Hungary, Romania, and the northern Balkans, but has not been reported from Greece (HORION, 1967, RANBOUSEK, 1910, and material examined). The easternmost records are from Novosibirsk, Russian Federation (first record) and from China, from where it was first reported by PACE (1998). For additional records see BOHÁĆ et al. (1993), BÜCHE (1994), FRANZ (1938), KOCH (1968), KÖHLER \& StuMPF (1992), Linke (1907, 1927), LoHSE (1967), PEeZ \& KAHLEN (1977), SCHOLZE et al. (1999), SIEDE (1992), VOGEL (1982), and ZERCHE (1980). A. umbrosa occurs in a wide range of open and woodland habitats. It has been observed in arable land, in xerothermous and in moist grassland, in sand pits, dune areas, gardens, swamps, bogs, coastal habitats, as well as in xerothermous and in damp forests, where adult beetles were found in various microhabitats: in all kinds of decaying organic matter (leaf litter, flood debris, mammal (also human) and bird excrements, mammal and bird carrion, dead insects and snails, compost, fungus), on vegetation, between roots of old trees, in fox dens, in burrows of ground squirrels, and in nests of sand martins (BÜCHE, 1994, FOWLER, 1888, FrANZ, 1938, HORTON, 1967, KOCH, 1968, LOHSE, 1967, PEEZ \& KAHLEN, 1977, WÖRNDLE, 1950, and material examined). A. umbrosa is apparently an active flyer; on numerous occasions, the species has been collected at windows, with car-nets, or swept from grass vegetation during the period from April through July, with most records in May and June (FOWLER, 1888, HORION, 1967, LINKE, 1927, LOHSE, 1967, VOGEL, 1982, WÖRNDLE, 1950, and material examined).

Various authors (e. g. HORION, 1967, LOHSE, 1967, 1984) have assumed that $A$. umbrosa is associated with subterranean burtows and nests of small mammals. However, this hypothesis has not been confirmed by systematic studies of such habitats (especially of mole nests). Morphological (slender body, massive antennae) and bionomic evidence (see below) suggests that the ecology of $A$. umbrosa and other congeners may be similar to that of Ilyobates KRAATZ, Callicerus GRAVENHORST, and Pseudosemiris MACHULKA, whose reproduction and hibernation habitats are unknown, too (AsSING, 1999, 2001).

The specimens examined were collected from February through August and in October (Fig. 15), with a maximum from April through August; PEEZ AND KAHLEN (1977) also observed adult beetles in September and November. Records from December and January are unknown. According to HORION (1967), teneral specimens appear in July and August. These observations suggest that the species is univoltine with spring reproduction and pre-imaginal development taking place in spring and early summer. The mature eggs of $A$. umbrosa are very large (approximately $0.6 \mathrm{~mm} \mathrm{long}$ ), so that the number of eggs laid per female is probably relatively low. 


\subsubsection{Amarochara beterogaster CAMERON, 1939}

Figs. 16-24

Amarochara (Lasiochara) beterogaster CAMERON, 1939: 574f.

Amarochara (Lasiochara) simlaensis CAMERON, 1939: 574f., syn. n.

Amarochara (Lasiochara) smetanai PACE, 1992: 282; syn. n.

\section{Types examined}

A. heterogaster. Lectotype o [teneral], here designated: Ghum dist. Rongdong Valley, VVI-1931, Dr. Cameron / Am. heterogaster Cam TYPE [handwritten] / M. Cameron. Bequest. B.M. 1955-147 / Lectotypus ơ Amarochara beterogaster Cameron, desig. V. Assing $2000(\mathrm{BMNH})$.

A. simlaensis: Syntypes: 1 \&: Fagu 8000', Simla Hills. / Dr. Cameron. 9.IX.1921 / A. simlaensis Cam TYPE [handwritten] / M. Cameron. Bequest. B.M. 1955-147 / Syntype o Amarochara simlaensis Cameron, rev. V. Assing 2000 / Amarochara heterogaster Cameron det. V. Assing 2000 (BMNH); 1 \% : same data, but "IX.1921" and without handwritten type label (BMNH).

A. smetanai: Holotype o: NEPAL, Khandbari District / Kuwapani 2100m, 15.IV.1982, A. \& Z. Smetana / HOLOTYPUS Amarochara smetanai m. det. R. Pace 1988 / Amarochara smetanai sp. n. det. R. Pace 1988 / Amarochara heterogaster Cameron det. V. Assing 2000 (MHNG). Paratype + : same data as holotype (MHNG).

\section{Comments}

The original description of Amarochara heterogaster CAMERON is based on an unspecified number of syntypes. As the possibility that more syntypes exist cannot be excluded, the male syntype in the Cameron collection is here designated as lectotype to preserve the present interpretation of the species. The coloration indicated in the original description may be misleading, since the type is evidently a teneral specimen.

The original description of $A$. simlaensis CAMERON is based on an unspecified number of syntypes, two of which were located in the BMNH. A lectotype is not designated, since both of these syntypes are females and the possibility that a male syntype exists cannot be excluded.

A comparison of the types of $A$. heterogaster, $A$. simlaensis, and $A$. smetanai yielded no convincing evidence that they should represent distinct species. The lectotype of $A$. beterogaster is apparently a nanistic male with a relatively small pronotum, but the aedeagus is identical with that of the holotype of $A$. smetanai. According to PACE (1992), A. smetanai is distinguished from $A$. simlaensis and $A$. beterogaster by the different coloration of antennomere I, by the relatively shorter antennomere III, and by the wider elytra. A comparative examination of the types, however, revealed that these differences do not exist; in the lectotype of $A$. beterogaster, the elytra are even wider in relation to the pronotum than in the types of $A$. smetanai. Consequently, both $A$. simlaensis CAMERON and $A$. smetanai $\mathrm{PACE}$ are here placed in the synonymy of $A$. heterogaster CAMERON. 

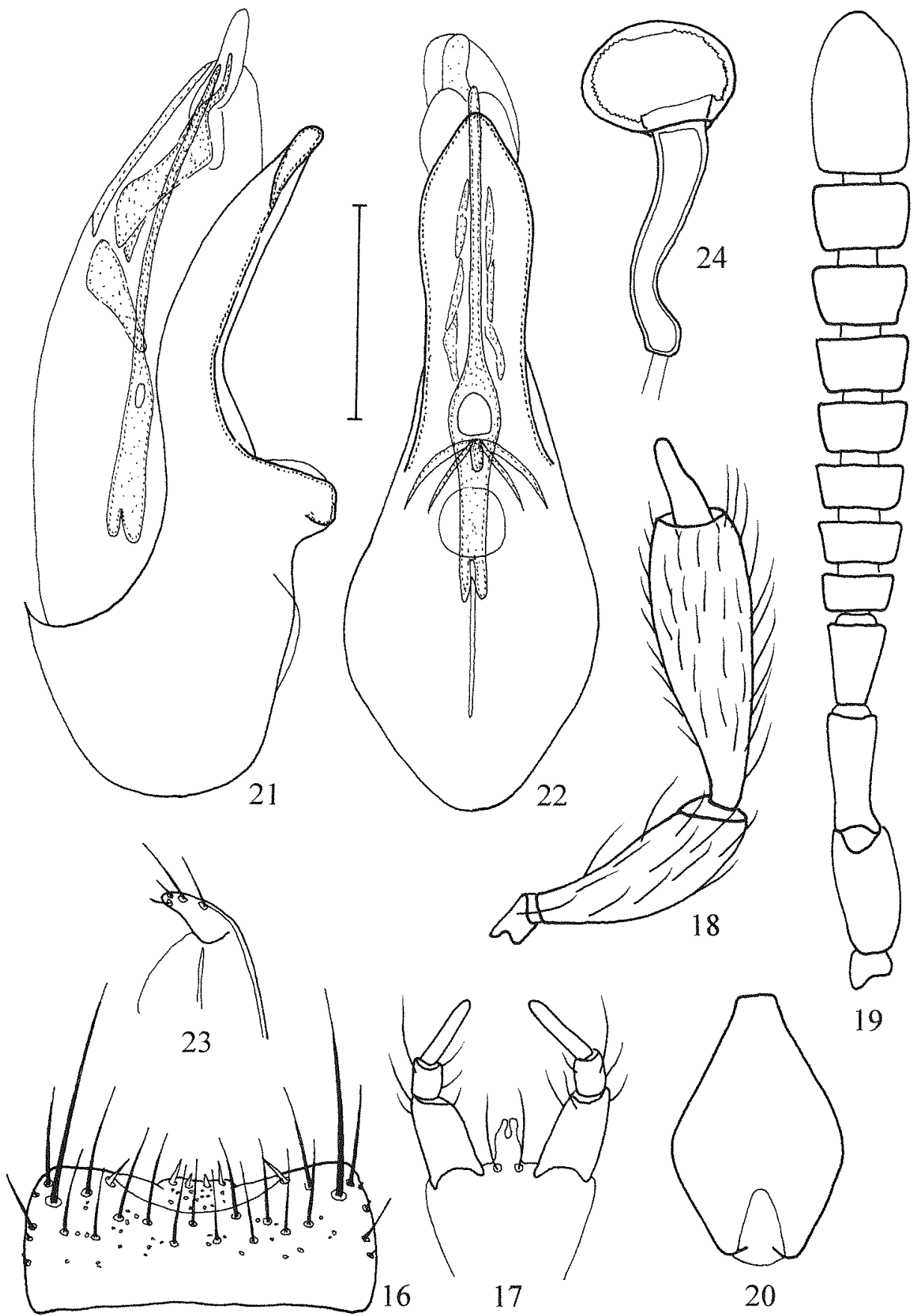

19

Figs. 16-24: Amarochara beterogaster CAMERON. Labrum (16); labium (17); maxillary palpus (18); antenna, pubescence omitted (19); tergum X, pubescence omitted (20); median lobe of aedeagus in lateral and in ventral view (21, 22); apical lobe of paramere (23); spermatheca (24). Scale: 16-18, 21-24:0.1 mm; 19-20: 0.2 mm. 


\section{Redescription}

2.6 - $3.4 \mathrm{~mm}$. Of similar external appearance as A. umbrosa. Forebody brown to dark brown, with the elytra slightly lighter; abdomen dark brown to blackish brown with the tergal hind margins lighter; antenna dark brown, with antennomeres I-III brown; legs brown; maxillary palpi light brown to dark brown. Head with variable, distinct to shallow, sometimes barely noticeable microsculpture; apical abdominal terga with very fine and shallow transverse microstriae; remainder of dorsal surface without microsculpture. Pubescence of forebody relatively short and dense (especially on pronotum).

Head of similar shape and morphology as in $A$. umbrosa, but less distinctly subquadrate, slightly (approximately 1.05 times) wider than long, not dilated behind eyes; postgenae relatively shorter, slightly longer than eyes; eyes large and convex, weakly projecting from lateral outline of head; postgenae weakly and smoothly convex, posterior angles obsolete. Puncturation extremely fine, barely noticeable. Genal carina rudimentary, usually visible only below eyes and/or near posterior constriction. Antenna more massive than in $A$. umbrosa, otherwise of similar morphology; antennomere I with shorter dorsoapical furrow than in average $A$. umbrosa; antennomere III shorter than II (Fig. 19). Mouthparts as in $A$. umbrosa, but ligula relatively larger (Fig. 17), labrum with median pair of sensilla at anterior margin stouter, and lateral margins of labrum with some very short setae (Fig. 16); maxillary palpus as in Fig. 18.

Pronotum of similar relative size and proportions as in $A$. umbrosa, weakly transverse, approximately $1.05-1.10$ times as wide as long and 1.13 - 1.25 times as wide as head; maximal width in anterior half; puncturation dense, but shallow, fine, and ill-defined. Mesosternum without median carina; mesosternal process apically relatively broadly truncate. Elytra at suture approximately 0.9 times the length of pronotum and approximately 1.3 times as wide as pronotum; posterior margin near posterior angles not distinctly sinuate; puncturation fine (but more distinct than that of head and pronotum), indistinctly granulose, and relatively dense. Legs as in A. umbrosa; first metatarsomere of variable length, approximately as long as the combined length of the two following tarsomeres or slightly longer.

Abdomen with anterior impressions of terga III-V slightly deeper and broader than in A. umbrosa; tergum VI without anterior impression; sterna III-V anteriorly constricted; puncturation in anterior impressions of terga III-V and near anterior margin of tergum VI very dense and coarse; on remainder of tergal surface, too, with numerous coarse (though somewhat less dense) punctures, which are decreasing in size and density towards the posterior tergal margins. Tergum $\mathrm{X}$ anteriorly broadly truncate, not distinctly elongated (Fig. 20); practically all of its surface, except for a small median area near hind margin, with very dense stout and long setae.

$\sigma^{*}$ : posterior margin of tergum VIII weakly convex, with a row of long and rather stout marginal setae; posterior margin of sternum VIII strongly convex, with a dense row of relatively long marginal setae and with interspersed long submarginal setae; aedeagus with long and slender ventral process, which is distinctly separated from the dorsal part of the median lobe; crista apicalis small, crista proximalis long, but not very prominent; internal sac with very long tube and with additional pair of long, weakly sclerotized structures (Figs. 21-22); apical lobe of paramere with two long and two short setae (Fig. 23). 
i: tergum VIII posteriorly almost truncate and with sparse and moderately long marginal setae; posterior margin of sternum VIII convex, row of marginal setae not distinctly modified, moderately short, denser and somewhat shorter in the middle than in lateral parts of posterior margin; spermatheca smaller than in $A$. umbrosa and with shorter duct (Fig. 24).

\section{Comparative and systematic notes}

For distinction from the similar $A$. umbrosa see description above. From other Himalayan species, except for $A$. sororcula, $A$. beterogaster differs in the absence of a distinct neck. Apparently based on the presence of fine (but rudimentary) genal carinae, CAMERON (1939) attributed the species to the subgenus Lasiochara. However, as can be inferred from other characters, the species is more closely related to $A$. umbrosa, the type species of the nominal subgenus, than to $A$. bonnairei, the type species of Lasiocbara (see section 3.3).

\section{Distribution and bionomics}

A. beterogaster is known from northern India (Himachal Pradesh and Ghum district near the border to Sikkim) and from two localities in Nepal; the type locality of $A$. smetanai is in the Khandbari district in eastern Nepal, and PACE (1984) recorded the species (as $A$. simlaensis) also from Jaljale Himal. A. heterogaster was collected at altitudes between 2100 and $2950 \mathrm{~m}$ in spring (April through June) and in autumn (September, November).

\subsubsection{Amarochara sororcula CAMERON, 1939}

Figs. 25-28

Amarochara (Lasiochara) sororcula CAMERON, 1939: $574 \mathrm{f}$.

\section{Type examined}

Syntype $9:$ Dehra Dun. Dr. Cameron. 7-10-1922. / Dung / Am. sororcula Cam TYPE [handwritten] / M. Cameron. Bequest. B.M. 1955-147 / Syntypus Amarochara sororcula Cameron, rev. V. Assing 2000 (BMNH).

\section{Comments}

The original description is based on an unspecified number of syntypes. The possibility that a male syntype exists cannot be ruled out, so that a lectotype is not designated.

\section{Redescription}

Of similar size as $A$. umbrosa; $2.7 \mathrm{~mm}$. Head and abdomen (except for the lighter tergal margins) dark brown; pronotum brown; elytra yellowish brown; antennomeres I-II light brown and III-XI dark brown; legs and maxillary palpi testaceous. Mictosculpture as in A. umbrosa. Pubescence of forebody distinctly shorter than in $A$. umbrosa and \pm decumbent. 


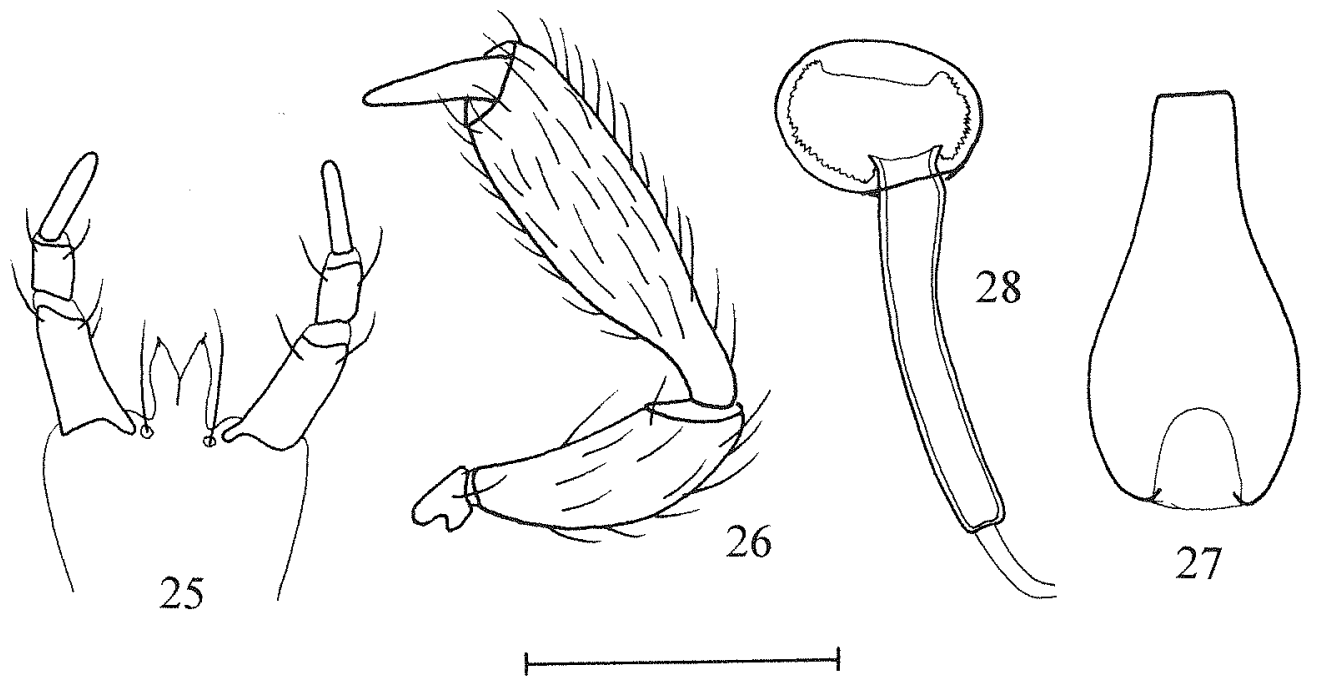

Figs. 25-28: Amarochara sororcula CAMERON. Labium (25); maxillary palpus (26); tergum X, pubescence omitted (27); spermatheca (28). Scale: $0.1 \mathrm{~mm}$.

Head of similar shape and size as in $A$. wmbrosa, but postgenae relatively shorter and posterior angles only weakly marked; eyes large, in dorsal view almost as long as postgenae; puncturation similar to that in $A$. umbrosa. Genal carinae present, but fine. Antenna as slender as in $A$. umbrosa and of similar morphology, but antennomere III slightly stouter. Mouthparts as in Figs. 25-26.

Pronotum relatively large, 1.3 times as wide as head and 1.15 times as wide as long; maximal width near the middle, posterior angles weakly marked; puncturation slightly denser than in $A$. umbrosa.

Elytra only slightly (1.15 times) wider than and at suture 0.85 times as long as pronotum; posterior margins near posterior angles not sinuate; puncturation fine and dense, distinctly denser than in A. umbrosa. Mesosternum apically acute. Legs slightly shorter than in $A$. umbrosa, metatarsus almost as long as metatibia; first metatarsomere almost as long as the combined length of the three following tarsomeres.

Abdomen with anterior impressions of terga III- $V$ shallow and posteriorly ill-defined; sterna III-V with weak anterior constriction; puncturation distinctive: on terga III-VI weakly granulose; on terga III-V dense and well-defined, but not very coarse, not coarser and denser in anterior impressions than on remainder of tergal surface; on tergum VI slightly coarser and denser than on terga III-V, and on tergum VII finer and sparser than on terga III-VI. Tergum X apically elongated and broadly truncate (Fig. 27); setae testaceous and stout, in anterior half very dense (also in the middle); posterior median area without setae. 
o: unknown.

क: tergum VIII posteriorly weakly convex and with row of relatively sparse and long marginal setae; posterior margin of sternum VIII weakly convex and with moderately dense row of weakly modified marginal setae; spermatheca of similar size and morphology as in A. umbrosa, but capsule somewhat less globulous (Fig. 28).

\section{Comparative and systematic notes}

From $A$. umbrosa, $A$. sororcula is readily separated especially by the presence of genal carinae, the less marked posterior angles of the head, the relatively larger pronotum, the short, dense, and decumbent pubescence of the forebody, the shallower anterior impressions of the abdominal terga $I I I-V$, and by the characteristic puncturation of the abdomen. From other Palaearctic congeners, it differs in the slender antennae, the short, dense, and decumbent pubescence, the shallow anterior impressions of the abdominal terga III-V, and in the puncturation of the abdomen. Apparently based on the presence fine genal carinae, CAMERON (1939) attributed the species to the subgenus Lasiochara. However, as can be inferred from other characters, the species is more closely related to A. umbrosa, the type species of the nominal subgenus, than to $A$. bonnairei, the type species of Lasiochara (see section 3.3).

\section{Distribution and bionomics}

Only the holotype of this species has become known, which was collected from dung in Dehra Dun (Northern India: Uttar Pradesh).

\subsubsection{Amarochara inquilina (CASEY, 1906)}

Figs. 38, 43

Nasirema inquilina CASEY, 1906: 311.

Amarochara (s. str.) inquilina: BERNHAUER \& SCHEERPELTZ (1926).

\section{Types examined}

Lectotype $P$ [dissected and remounted, with two workers of brown Formica sp. attached to the pin], here designated: Iowa City Wickham V-22-99/ 5-5-5 / inquilina Cay / CASEY bequest 1925 / TYPE USNM 39749 / Lectotype \& Nasirema inquilina Casey desig. V. Assing 2000 / Amarochara inquilina (Casey) det. V. Assing 2000 (USNM). Paralectotype + [with worker of brown Formica sp. attached to the pin]: Iowa City Wickham / Nest 6 4.19.99 / CASEY bequest 1925 / inquilina-PARATYPE 2 USNM 39749 / Paralectotype + Nasirema inquilina Casey desig. V. Assing 2000 / Amarochara inquilina (Casey) det. V. Assing 2000 (USNM).

\section{Comments}

The original description is based on an unspecified number of type specimens. In order to fix a single name-bearing type and to secure the present interpretation of the species, the syntype in better condition is here designated as lectotype. 


\section{Redescription}

In size and general appearance similar to $A$. umbrosa, but distinguished as follows:

Coloration lighter, whole body \pm uniformly ferrugineous, with the anterior $3 / 4$ of abdominal tergum VI infuscate, and with the legs and the basal antennomeres testaceous. (In the paralectotype, the posterior part of the head is somewhat darker and the elytra are lighter than the pronotum.) Pubescence \pm decumbent.

Head with extremely weak, but (at higher magnifications) noticeable microsculpture. Head weakly (approximately 1.05 times) wider than long, larget in relation to pronotum than in A. umbrosa (see ratio below); hind angles very weakly marked, almost obsolete; eyes slightly smaller and less convex, only weakly protruding from lateral outline of head. Genal carina absent. Antenna of similar length as in A. umbrosa, but distinctly more massive; antennomere with distinct and rather long dorso-apical furrow, antennomere IV longer and less transverse, antennomeres VI-X much wider and more transverse than in $A$. umbrosa, more than twice as wide as long. Mouthparts of similar morpho$\operatorname{logy}$ as in A. umbrosa.

Pronotum 1.10 - 1.15 times as wide as long and only 1.20 times as wide as head; maximal width approximately halfway between middle and anterior angles; posterior angles obtuse, moderately marked; puncturation extremely fine; microsculpture similar to that of head or barely noticeable.

Elytra at suture approximately 0.9 times the length of pronotum and approximately 1.25 times as wide as pronotum, distinctly shorter in relation to pronotum than in $A$. umbrosa; posterior margin near posterior angles not or very indistinctly sinuate. Legs slightly longer than in $A$. umbrosa, first metatarsomere slightly or distinctly longer than the combined length of the two following tarsomeres. Mesosternum without median carina; mesosternal process apically acute.

Abdomen as in $A$. umbrosa, but puncturation on the whole finer, in anterior impressions of terga III-V moderately coarse and on remainder of dorsal surface very fine; tergum $\mathrm{X}$ of similar chaetotaxy as in $A$. umbrosa, but anteriotly less elongate (Fig. 43).

o: unknown.

q: posterior margin of tergum VIII truncate, with row of sparse marginal setae; posterior margin of sternum VIII weakly convex, marginal setae weakly modified, distinctly denser in the middle than laterally; spermatheca larger than in $A$. umbrosa (Fig. 38).

\section{Comparative notes}

From $A$. umbrosa, A. inquilina differs as indicated in the redescription abore. For distinction from other North American congeners see comparative notes and descriptions below.

\section{Distribution and bionomics}

The species is known only from the type locality in Iowa, where it was collected in ant nests (Formica sp.). 


\subsubsection{Amarochara fenyesi BLATCHLEY, 1910}

Figs. 29-37

Amarochara fenyesi BLATCHLEY, 1910: 361.

\section{Type examined}

Syntype 9: TYPE / Clark Co., Ind. W. S. B., 6' - 6-09/3594 det. A. Fenyes / Purdue Blatchley collection / Amarochara fenyesi sp. nov. 5996 / Amarochara fenyesi Blatchley det. V. Assing (PU).

\section{Additional material examined}

Kansas: 10 , Douglas $3.2 \mathrm{~km}$ N Baldwin, Breidenthal Reserve, 21.-29.V.1996, M. Panaras KAN 1P96 008, ex: flight intercept trap (UKNHM); $30^{*} \sigma^{*}, 2$ 우, Douglas $3.2 \mathrm{~km} \mathrm{~N}$ Baldwin, Breidenthal Reserve, 10 17.VII.1996, M. Panaras KAN 1P96 037, ex: flight intercept trap (UKNHM, cAss); 10 , 1 \% , same data, but 3.-10.VII.1996, KAN 1P96 034 (UKNHM, cAss); 107, same data, but 4.-7.VI.1996, KAN 1P96 010 (UKNHM); 2 o 9 , same data, but 14.-18.VI.1996, KAN 1 P96 019 (UKNHM); 1 o [?], same data, but 18.25.VI.1996, KAN 1P96 025 (UKNHM); 10, same data, but 7.-11.VI.1996, KAN 1 P96 016 (UKNHM); 10 , same data, but 17.-25.VII.1996, KAN 1 P96 041 (cAss). Georgia: 1 \& [identified by Fenyes as Nasirema inquilina CASEY], date and locality not specified (DEI).

\section{Description}

In general appearance similar to $A$. umbrosa, but distinguished as follows:

Body larger, $3.0-3.8 \mathrm{~mm}$ (abdomen fully extended). Coloration on average lighter than in A. umbrosa; head, antennae, and abdomen dark brown to blackish, with the tergal margins, the abdominal apex and sometimes the basal antennomeres lighter, ferrugineous to castaneous; pronotum castaneous to dark brown; elytra light brown; legs testaceous; maxillary palpi testaceous or infuscate. Microsculpture similar to that in $A$. umbrosa.

Head of similar shape and morphology as in $A$. umbrosa, but eyes relatively larger, in dorsal view approximately as long as postgenae or nearly so. Puncturation as in $A$. umbrosa. Genal carina absent. Antenna more massive than in $A$. umbrosa, antennomeres IV and V more transverse, and VI-X wider; dorso-apical furrow of antennomere I variable, usually pronounced (Fig. 37). Mouthparts as in A. umbrosa, but third joint of maxillary palpus longer and more slender (Fig. 34); labium as in Fig. 33.

Pronotum larger (especially in relation to head) than in $A$. umbrosa, approximately as wide as long and 1.25 - 1.3 times as wide as head; maximal width near the middle; puncturation very fine and sparser than in average $A$. umbrosa.

Elytra at suture 0.75 times the length of pronotum and approximately 1.25 times as wide as pronotum, distinctly shorter in relation to pronotum than in $A$. umbrosa, posterior margin near posterior angles weakly sinuate; puncturation similar to that in $A$. umbrosa. Legs longer and more slender than in $A$. umbrosa, first metatarsomere approximately as long as the combined length of the three following tarsomeres (Fig. 35). Mesosternum without median carina; mesosternal process apically \pm narrowly truncate or rounded, almost acute.

Abdomen as in A. umbrosa, but puncturation in anterior impressions of terga III-V and on remainder of dorsal surface coarser and sparser, integument with more shine; tergum $\mathrm{X}$ of similar chaetotaxy as in $A$. umbrosa, but anteriorly more strongly elongated 
(Fig. 36), at least in 9 occasionally with 2-4 setae near posterior membranous fringe. 0 : posterior margin of tergum VIII \pm truncate, that of sternum VIII weakly convex to almost truncate and with row of rather dense and long marginal setae; median lobe of aedeagus of similar general morphology as in $A$. umbrosa, but larger, more slender, with much longer ventral process, and with distinct crista apicalis and crista proximalis (Figs. 29-30); apical lobe of paramere short, broadly triangular, and with rather long setae (Fig. 31).

P: posterior margin of sternum VIII moderately convex, row of marginal setae very weakly modified, relatively thin; spermatheca of similar absolute, but of lower relative size than in A. umbrosa (Fig. 32).

\section{Comments and comparative notes}

The original description is based on material from "Lawrence and Clark counties", so that it can be assumed that Blatchley had at least two syntypes before him. Since the type
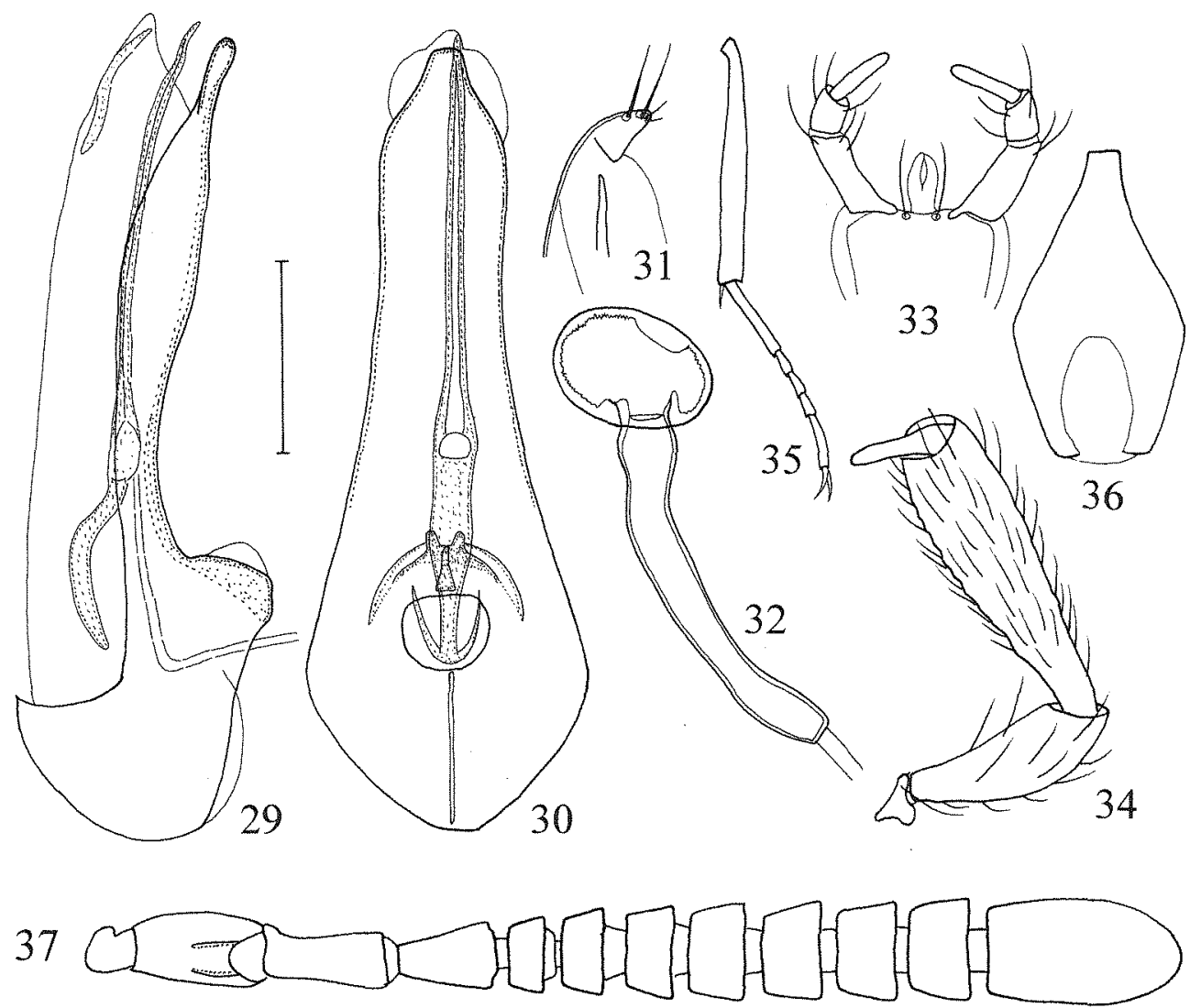

Figs. 29-37: Amarocbara fenyesi BLATCHLEY. Median lobe of aedeagus in lateral and in ventral view $(29,30)$; apical lobe of paramere (31); spermatheca (32); labium (33); maxillary palpus (34); metatibia and metatarsus, pubescence omitted (35); tergum X, pubescence omitted (36); antenna, pubescence omitted (37). Scale: 29-34: $0.1 \mathrm{~mm} ; 36-37: 0.2 \mathrm{~mm} ; 35: 0.4 \mathrm{~mm}$. 
specimen in the Blatchley collection is a female, it is not designated as lectotype; a male syntype may yet be discovered. Apparently, either the date indicated in the original description ("May 6-May 12") or that given on the label (June 6) is erroneous. Nevertheless, there is little doubt that the examined specimen from the Blatchley collection in fact represents a syntype.

The specimen from Georgia is of somewhat lighter coloration and its pronotum is even larger than in the material from Kansas. Since the examined syntype is a female, it is not absolutely certain that it is conspecific with the specimens listed as additional material examined. However, significant distinguishing characters were found neither in external morphology nor in the female primary and secondary sexual characters, so that they are here attributed to the same species.

From the similar $A$. umbrosa, $A$. fenyesi is readily distinguished especially by its larger size, the more massive antennae, the larger pronotum, the relatively shorter elytra, the longer first metatarsomere, and by the coarser puncturation of the abdomen (see description above). The North American A. inquilina is smaller, of lighter coloration, and has much shorter antennae, a more transverse and distinctly smaller pronotum (especially in relation to head), longer elytra, a much finer abdominal puncturation, and a larger spermatheca.

\section{Distribution and bionomics}

The species is known from Indiana, Kansas, and Georgia. Most of the specimens were collected with flight intercept traps between 21 May and 25 July (maximum in July), suggesting that $A$. fenyesi is an active flyer and more widespread than is currently known.

\subsubsection{Amarochara brevios sp. $\mathrm{n}$.}

Figs. $39-42$

\section{Types}

Holotype o : USA: Kansas; Douglas $3.2 \mathrm{~km} N$ Baldwin, Breidenthal Reserve, 10-25 July 1996, KAN 1 P96 047, M. Panaras, ex: flight intercept trap / Holotypus o Amarocbara brevios sp. n. det. V. Assing 2000 (UKNHM).

Paratypes: 1 \%: USA: Kansas; Douglas $3.2 \mathrm{~km}$ N Baldwin, Breidenthal Reserve, 10-17 July 1996, M. Panaras KAN 1P96 037, ex: flight intercept trap (cAss); $10^{\text {* }}$ : same data, but 3-10 July 1996, KAN 1P96 032C (UKNHM).

\section{Description}

In size and general appearance highly similar to $A$. umbrosa, but distinguished as follows. Length of holotype: $2.8-3.2 \mathrm{~mm}$. Coloration lighter than in $A$. umbrosa; head and abdomen dark brown, with margins of the abdominal terga and the abdominal apex lighter; pronotum brown, elytra light brown; legs and maxillary palpi testaceous; antennae bicoloured, dark brown, with the basal antennomeres yellowish to ferrugineous. Microsculpture similar to that in $A$. umbrosa. Pubescence relatively short and \pm decumbent.

Head of similar shape and morphology as in A. umbrosa, but posterior angles less marked. Antenna of similar length as in $A$. umbrosa, but distinctly more massive; antennomere I in the holotype with very short and weak dorso-apical furrow, antennomere III stouter and apically more strongly dilated than in $A$. umbrasa; antennomeres VI-IX wider 
and more transverse (distinctly more than twice as wide as long, antennomere VI almost three times as wide as long) than in $A$. umbrosa. Genal carina absent.

Pronotum and elytra of similar size, shape, and proportions as in $A$. umbrosa; puncturation of pronotum very fine and rather dense. Legs of similar morphology as in $A$. umbrosa, first metatarsomere longer than the combined length of the two following tarsomeres, but shorter than the combined length of the three following tarsomeres; length of metatarsus approximately $0.3 \mathrm{~mm}$. Mesosternum without median carina; mesosternal process apically narrowly truncate.

Abdomen with dense and relatively coarse puncturation, on the whole similar to $A$. umbro$s a$, but coarser, especially on tergum VI; punctures finer near posterior than near anterior tergal margins. Tergum $\mathrm{X}$ of similar shape and chaetotaxy as in $A$. umbrosa.

$\sigma^{*}$ : sternum VIII posteriorly weakly convex and with dense long marginal setae; aedeagus with median lobe very similar to that in $A$. umbrosa, but ventral process with pronounced lateral folds and crista proximalis not reduced (Figs. 39-40); apical lobe of paramere with longer setae (Fig. 41).

q: posterior margin of tergum VIII truncate, that of sternum VIII weakly convex, row of marginal setae very weakly modified; spermatheca with larger capsule and longer duct than in A. umbrosa (Fig. 42).

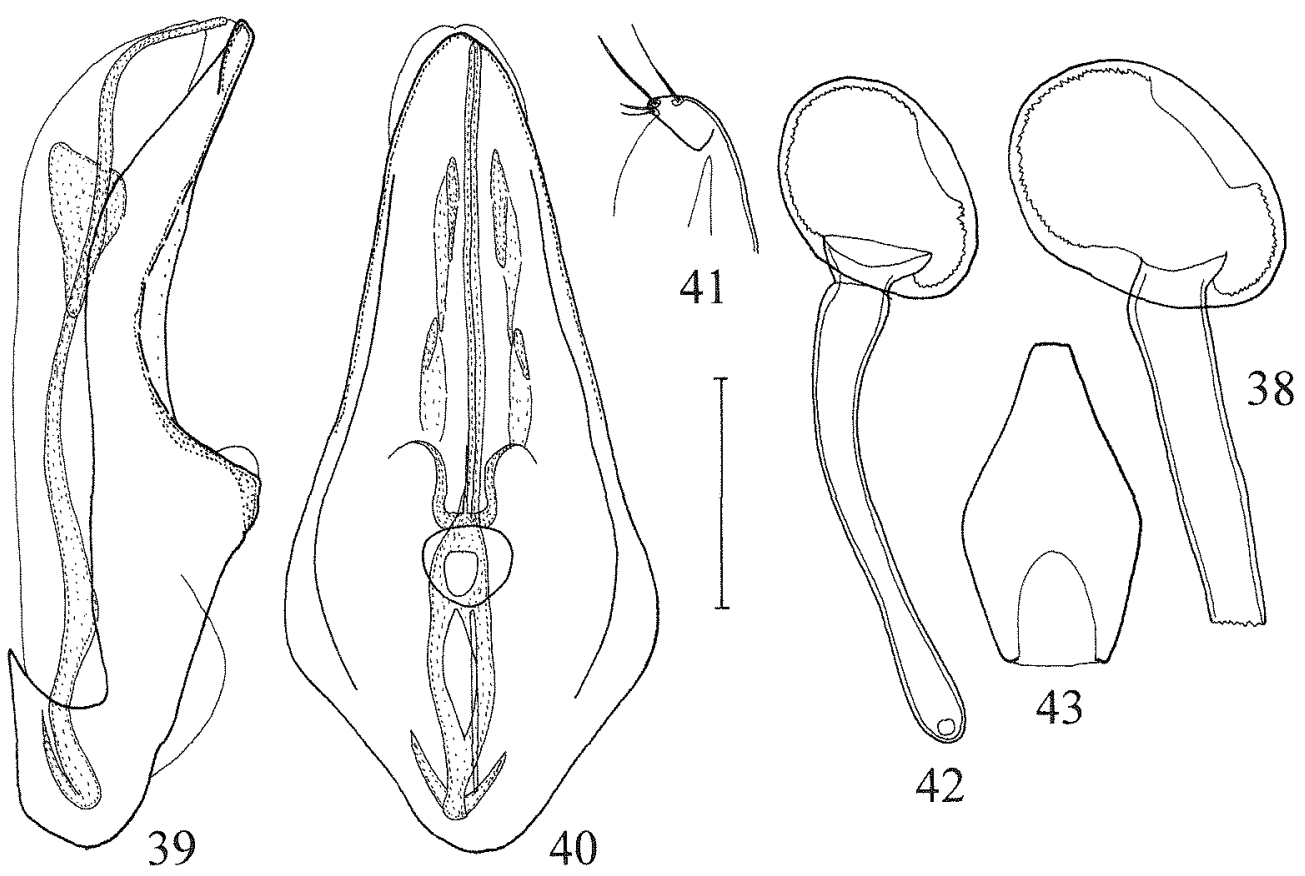

Figs. 38-43: Amarochara inquilina (CASEY) $(38,43)$ and A. brevios sp. n. (39-42). Spermatheca, duct incomplete (38); median lobe of aedeagus in lateral and in ventral view $(39,40)$; apical lobe of paramere (41); spermatheca (42); tergum $X$, pubescence omitted (43). Scale: 38-42:0.1 $\mathrm{mm} ; 43: 0.2 \mathrm{~mm}$. 
Derivatio nominis: The name is a noun in apposition composed of the Latin adjective brevis (short) and the Latin noun os (leg) and refers to the relatively short legs, which distinguish this species from other North American congeners.

\section{Comparative notes}

From the similar $A$. umbrosa, $A$. brevios is best distinguished especially by the lighter colour, the stouter antennae, and the different genitalia. The two other North American species, A. inquilina and $A$. fenyesi, differ in the longer legs, the different coloration, the distinctly sparser abdominal puncturation, the former in addition by the less convex eyes, the much finer abdominal puncturation, and the latter by larger size, a much larger pronotum, longer antennae, and a smaller spermatheca.

\section{Distribution and bionomics}

The species is known only from the type locality in Kansas, where it was collected with a flight intercept trap in July, together with $A$. fenyesi.

\subsection{The bonnairei group}

The bonnairei group includes at least 7 species, all of them occurring in the Western Palaearctic region (see also section 4.6). A. tingitana JARRIGE may belong to this group, too, but as the holotype is lost, its phylogenetic affiliations must remain doubtful. The position of $A$. crassicornis is somewhat uncertain, because the apical abdominal segments of the holotype were missing and no additional material was available for examination, so that the conditions of many significant characters are unknown.

The bonnairei group is relatively weakly defined, because most of the synapomorphies are either not realized in all the species of the group or because they also occur in other groups of Amarochara. In addition, the fact that the male sexual characters of two species are unknown renders a definition difficult.

The species of the bonnairei group share the following derived characters: relatively small eyes (exceptions: A. caeca, A. splendens, A. crassicomis), fine genal catinae (exception: $A$. splendens, A. crassicornis), long antennae, a slender third joint of the maxillary palpus, a large pronotum (exception: $A$. bonnairez), a long and slender first metatarsomere, a fine and sparse puncturation of the posterior halves of the abdominal terga III-VI (relatively coarse and dense in $A$. splendens), an anteriorly distinctly elongated tergum $\mathrm{X}$, an aedeagus with the ventral process distinctly separated from the main body of the aedeagus, a moderately or very long basal part of the internal tube of the aedeagus, and a relatively large spermatheca (see section 3.3.1). 


\subsubsection{Amarochara bonnairei (FAUVEL, 1865), nomen protectum}

Figs. 44-51, Map 3

Calodera (Ihobates) bonnairii [sic] FAUVEd, 1865: 287.

Oxypoda glabriventris RYE, 1865: 212; nomen oblitum.

Ilyobates (Mniobates) bonnairei: MULSANT \& REY (1875).

Amarochara (Lasiochara) bonnairei: GANGLBAUER (1895).

\section{Types examined}

Calodera bonnairei: Neotype o , here designated: Dijon Rouget / Coll. et det. A. Fauvel A. Bonnairei Fvl R.I.Sc.N.B. 17.479 / Neotypus Calodera bonnairei Fauvel desig. V. Assing 2000 / Amarochara bonnairei (Fauvel) det. V. Assing 2000 (IRSNB).

Oxypoda glabriventris: Lectotype + , here designated: Power. Mickleham / glabriventris, Rye / bonnairei Fvl. / W.A.Power B.M.1896-69 / ? Syntype / Lectotypus o Amarochara glabriventris (Rye), desig. V. Assing 2000 (BMNH). Paralectotypes: 2 o f : Power. Mickleham / W.A.Power B.M.1896-69 / ? Syntype / Paralectotypus of Amarochara glabriventris (Rye), desig. V. Assing 2000 (BMNH).

\section{Comments}

The original description of C. bonnairei FAUVEL is based on a "seul exemplaire" collected by M. Bonnaire "aux environs de Paris"; the author expressly thanks M. Bonnaite for the gift of the holotype. In the Fauvel collection at the IRSNB, four specimens of $A$. bonnairei were found: one from Marly, another one from Dijon, and two examples from Metz. Thus, the holotype is either lost or it was given away by Fauvel, who was in the habit of exchanging material with other coleopterists. If the latter is the case, there is little chance of ever recovering and identifying the holotype. In view of the similarity of Amarochara species and in order to preserve the long-standing interpretation of the species by fixing a single name-bearing type, the specimen from Dijon in the Fauvel collection is here designated as the neotype of the species.

FAUVEL (1865) described $A$. bonnairei as Calodera bonnairii, but dedicated the species to M. Bonnaire. The name was treated by subsequent authors as an incorrect original spelling and emendated to bonnairei, which is in accordance with Articles 32.5.1. and 33.2.2. of the ICZN (1999).

The original description of Oxypoda glabriventris RYE is based on "several examples ... taken in May and June, 1863, by Dr. Power ... in Jeadly Lane, Mickleham, Surrey". Both the locality and the collector are in agreement with the data indicated on the labels of the specimens listed above. In order to secure the long-standing synonymy with $A$. bonnairei and to fix a single name-bearing type, a syntype in good condition was selected as lectotype.

Both A. bonnairei (FAUVEL) and A. glabriventris (RYE) were described in 1865. As explicitly stated above the description of $A$. glabriventris, it was published in February of that year, whereas there is no indication in what month the description of $A$. bonnairei appeared in print. Thus, according to the ICZN (1999), December 1865 must be assumed as the publication date and, consequently, A. bonnairei is the junior synonym. Nevertheless, this name is here preserved and given precedence according to Article 23.9 of the current International Code of Zoological Nomenclature (ICZN, 1999). As will be shown, 
both conditions for the application of Article 23.9 are met. To my knowledge, the senior synonym Oxypoda glabriventris has not been used as a valid name after 1899. A. bonnairei (FAUVEL), in contrast, has been used as the valid name in a number of works by clearly more than 10 authors in the past 50 years. In order to fulfil the requirements of the Code, a selection of 27 publications, in which $A$. bonnairei is used as the valid name of the species in question, is here listed: AssING (1994b), BOHÁČ et al. (1993), BÖHME (1996), FrANZ (1970), GEISER (1998), GÜRIICH et al. (1995), HANSEN (1996), HANSEN et al. (1995), HORION $(1951,1967)$, KOCH (1974, 1989), KÖHLER (2000), KÖHLER \& KLAUSNITZER (1998), LIKOVSKÝ (1985), LINKE (1962), LOHSE (1967, 1974), LUCHT (1987), LuNDBERG (1995), MAJZLAN \& JÁSZAY (1997), PALM (1972), SCHEERPELTZ (1968), SCHMAUS (1969), SCHÜLKE et al. (1992), ZANETTI (1995), ZIEGLER et al. (1994). In conclusion, based on Article 23.9 of the Code, the following synonymy is established: Calodera bonnairei FAUVEL, 1865 (nomen protectum) = Oxypoda glabriventris RYE, 1865 (nomen oblitum).

\section{Additional material examined}

France: Picardie: 1 ex., Orry-la-Ville, pond, 21.VI.1949 (cAss). Île-de-France: 1 ex., Paris (DEI); *1 ex., Chamarande, VI.1986, leg. Fongond (cTro); *1 ex., Les Essatts-le-Roi, UV-light,VI.1990, leg. Fongond (cTro). Nord-Pas-de-Calais: 1 ex., Marly (IRSNB); 1 ex., Marly, leg. Brisout (NHMW). Lorraine: 2 exx., Metz (IRSNB). Locality not specified: 1 ex. (DEI).

Germany: Schleswig-Holstein: 1 ex., Eutin, Kellersee, Kalkhütte, rotting alder trunk, V.1916 (DED); 1 ex., same data, but VII.1916 (DEI); 1 ex., Eutin, Kalkhütte, 25.V.1920 (DEI). Niedersachsen: *1 ex., Hildesheim-Ochtersum, Trillkegut, pitfall trap, VII.2001, leg. Sprick (cAss). Nordrhein-Westfalen: 2 exx., Kleve, Reichswald, NWZ Rehsol, 19.VII.1995, leg. Köhler (cKöh, cWun); 2 exx., Kleve Reichswald, NWZ Geldenberg, 23.V.1995, leg. Köhler (cKöh, cAss); 1 ex., same data, but 23.VIII.1995 (cKöh); 1 ex., Jülich, Hambach, Lindenberger Wald, 28.VII.1991, leg. Köhler (cKöh); 4 exx., Hellberg near Scherfede, 24.V.1999, leg. Köhler (cKöh, cAss); 30 $0^{*}, 6$ 우 , Porta Westfalica, Wittekindsbetg, window trap, 2.VII.1992, leg. Borcherding (cAss, cWun); 19 , same data, 21.VI.1992 (cAss). Rheinland-Pfalz: $10^{\star}$, Saarburg/Saar, Taben-Rodt, car-net, 6.VI.1996, leg. Renner (cRen). Bayern: 1 ex., Kelheim, Hienheimer Forst, Buchberg, 1993 (cKöh); 1 ex., Kelheim, Hienheimer Forst, 1995, leg. Schubert (cKöh). Sachsen-Anhalt: 3 exx., Mößlitz near Zörbig, 18.\&21.VII.1955, leg. Dorn (MNHUB); 1 ex., Harz, Bodetal, 6.VII.1941, leg. Ihssen (MNHUB). Thüringen: 19, Ichstedt, Lindenallee, 16.VI.1996, leg. Grimm (cVog). Sachsen: 4 exx., Leipzig, Stötteritz, 12.IX.1956, leg. Linke (SMTD); 1 \% , Leipzig, park, sweep-net, 31.V.1979, leg. Kieberlein (cVog).

Switzerland: Zürich: 1 ex., Zürich, Shlwald, 700-800m, 24.V.-5.VI.1996, leg. Schiegg (cKap). Bern: 1 ex., Aarberg, VI.1950, leg. Linder (NHMW). Genève: 1 ex., Cartigny, VI.1976, leg. Toumayeff (MHNG).

Austria: Niederösterreich: 1 ex., Hainburg, Braunsberg, dry grassland, pitfall, 31.V.1996 (cKap); 1 ex., Semmering, leg. Strupi (NHMW); 1 ex., Vöslau (NHMB). Steiermark or Niederösterreich: 2 exx., Wechsel, 1889, leg. Ganglbauer (NHMW).

Italy: Friuli-Venezia Guilia: 1 ex., Trieste, Basovizza-Opcina, leg: Moczarski \& Scheerpeltz (NHMW). Abruzzo: $1 \sigma^{\star}, 20$ exx., Prov. di l’Aquila, $10 \mathrm{~km}$ SE Castel di Sangro, Mte. Pagano, leg. Paganetti (DEI, FIS, MHNG, MNHUB, NHMB, NHMW, NMP, SMNS, cAss); 3 exx., Castel di Sangro, leg. Paganetti (DEI, NHMW, SMTD). Basilicata: $10^{7}, 1$ \% , Pietrapertosa, Mt. Impiso (PZ), 6.VII.1987, leg. Angelini (cAss); 1 \%, La Maddalena, Abricola (PZ), 1400m, 7.-9.VII.1987, leg. Angelini (cWun).

Czech Republic: Bohemia: 1 ex., S Praha, Vrané nad Vltavou (NMP); 1 ex., Závist neat Praha, 10.VI.1888 (NHMW).

Slovakia: 1 ơ, 2 exx., Košice, 1927, leg. Machulka (NMP, cAss); 1 ex., E Banská Bystrica, Slorenska Lupča, 7.VI.1995, leg. Behne (DEI); 10 , Teplý Vtch, 2.V.1998, leg. Smatana (cSch); 1 \%, "SO-Slowakei", 2.-10.V.1967, leg. Vogt (FIS).

Hungary: 1 ex., Zirč , 18.V.1985, leg. Sieber (cVog).

Bosnia-Herzegovina: 2 exx., Maklen Pass, 1902, leg. Leonhard (DEI, NHMW). 
Turkey: 4 exx., ca. $20 \mathrm{~km}$ NE Istanbul, Alem Dagh, leg. Bodemeyer (DEI, NHMW, cAss); $10^{*}$, Sinop, 23 km N Boyabat, S Bektas, 20.V.1976, leg. Besuchet \& Löbl (cAss).

Locality not specified: 2 exx. (NHMW).

\section{Redescription}

$2.8-4.0 \mathrm{~mm}$ (abdomen fully extended). Colour of body usually yellowish brown to light brown, with the head, antennomeres IV-XI, the anterior central areas of the abdominal terga VI-VII, sometimes also of terga IV-V, often slightly darker brown; legs and mouthparts testaceous. Microsculpture of head and pronotum shallow, but usually distinct, composed of meshes of variable length and orientation; abdominal terga VI-VIII or VVIII usually with extremely weak transverse microsculpture or traces of such microsculpture. Pubescence of forebody relatively long and suberect.

Head of ovoid shape, slightly (approximately 1.10 times) wider than long; noticeably dilated behind eyes, maximal width therefore not across eyes, but at some distance behind eyes; eyes moderately large, weakly convex, not distinctly projecting from lateral outline of head, only little more than half the length of postgenae in dorsal view, the latter smoothly curved towards posterior constriction. Puncturation very fine and sparse. Genal carina present, but fine. Antenna as in Fig. 50, antennomere I with dorso-apical

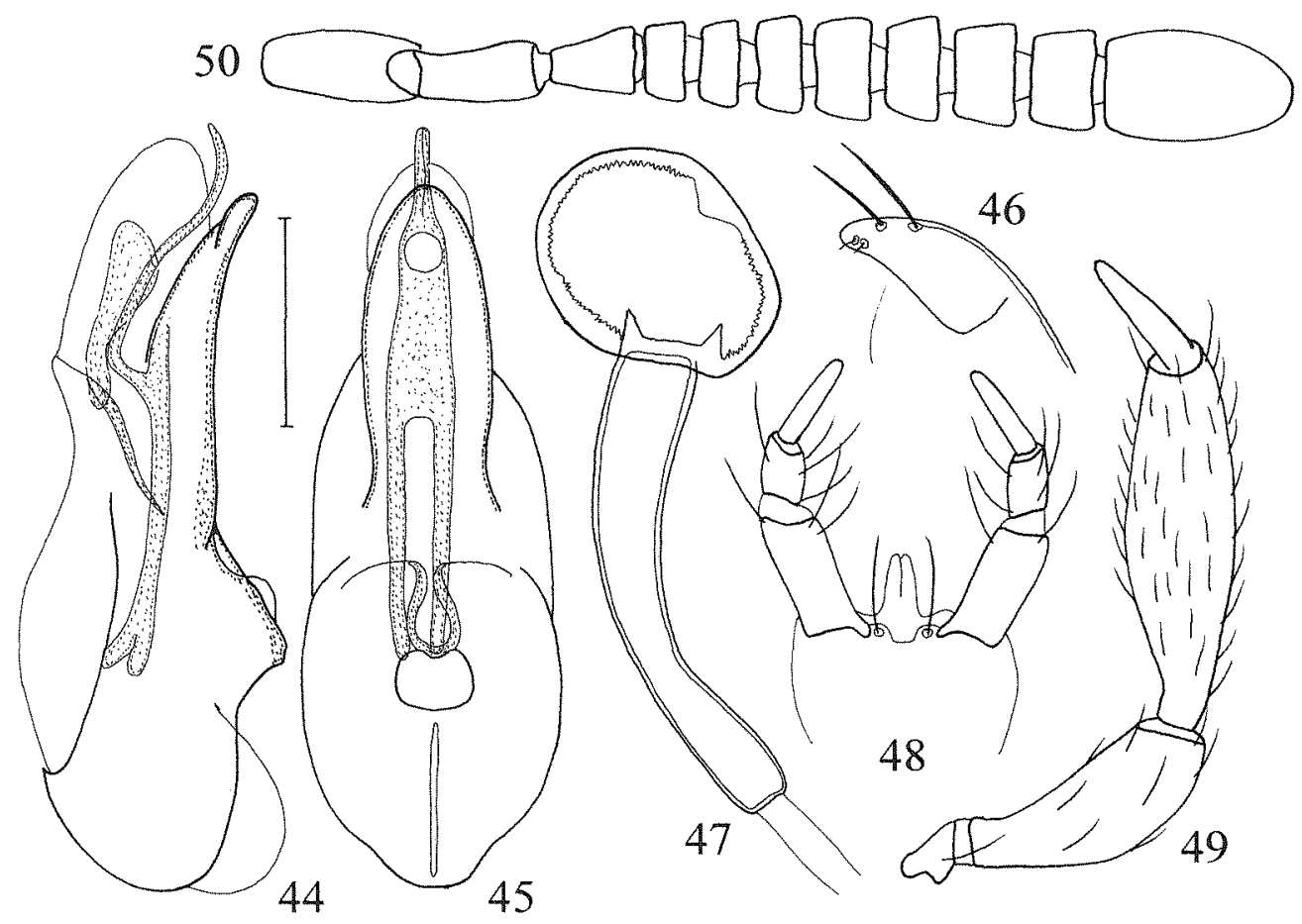

Figs. 44-50: Amarochara bonnairei (FAUVEL). Median lobe of aedeagus in lateral and in ventral view (44, 45); apical lobe of paramere (46); spermatheca (47); labium (48); maxillary palpus (49); antenna, pubescence omitted (50). Scale: 46-49:0.1 mm; 44-45: 0.125 mm; 50:0.2 $\mathrm{mm}$. 
furrow less pronounced than in $A$. umbrosa. Third joint of maxillary palpus very long and slender, more so than in $A$. umbrosa (Fig. 49); labium as in Fig. 48.

Pronotum approximately 1.1 times as wide as long and 1.2-1.3 times as wide as head; maximal width in or near the middle; puncturation similar to that of head or slightly denser and more distinct.

Elytra relatively shorter than in $A$. umbrosa, at suture $0.74-0.80$ times the length of pronotum and approximately 1.20 times as wide as pronotum; posterior margin near posterior angles weakly sinuate; puncturation weakly granulose, more distinct and somewhat denser than that of pronotum. Hind wings fully developed. Legs long and slender, first metatarsomere approximately as long as the combined length of the three following tarsomeres. Mesosternum with microsculpture similar to that in A. umbrosa, without median carina; mesosternal process acute.

Abdominal terga III-V with moderately deep, tergum VI without appreciable anterior impression; sterna III-V anteriorly constricted; puncturation fine, but distinct, denser and slightly coarser near anterior margin than on posterior part of terga; tergum $\mathrm{X}$ similar to that in the following species (cf. Fig. 56).

$\sigma^{\mathrm{t}}$ : posterior margin of tergum VIII slightly convex, with a row of moderately long and a sparse row of long thin setae; posterior margin of sternum VIII distinctly convex, with a dense row of relatively short marginal and a sparse row of long submarginal thin setae; aedeagus with ventral process of median lobe distinctly separated, in ventral view slender, in lateral view almost straight, with small basal fold, weak crista apicalis, and with pronounced crista proximalis; internal tube with very long basal part (Figs. 44-45); apical lobe of paramere as in Fig. 46.

$q$ : tergum VIII of similar shape and chaetotaxy as in 0 ; posterior margin of sternum VIII weakly convex, row of marginal setae very weakly modified, relatively thin, and very short; spermatheca as in Fig. 47.

\section{Comparative notes}

A. bonnairei is readily distinguished from $A$. umbrosa especially by larger size, lighter colour, the different shape of the head, the smaller and less prominent eyes, the more transverse antennomere VI, the more slender maxillary palpi, the presence of genal carinae, the microsculpture on head and pronotum, the more transverse and differently shaped pronotum, the shorter elytra, and the different primary and secondary sexual characters. For distinction from other species of Amarochara, especially the similar $A$. cribripennis (MULSANT \& REY), see the following species sections and the key in section 5. For systematic comments see remarks below the following species and section 3.3.

\section{Distribution and bionomics}

The area of distribution of $A$. bonnairei, evidently a very rare and local Ponto-Mediterranean element sensu LATTIN (1967), ranges from France and southern England in the west and northwest to southern Italy, the Balkans, and northern Anatolia in the south and southeast (Map 3). It seems to have been collected only once in Denmark and on very rare occasions in England. In France, it is known from several localities in the northern, eastern, central, and southeastern parts. In Germany, it has been recorded from the following regions: Bayern, Baden-Württemberg, Hessen, Rheinland-Pfalz, 
Nordrhein-Westfalen, Niedersachsen (first confirmed record), Schleswig-Holstein, Sachsen-Anhalt, Brandenburg, Thüringen, and Sachsen. It has been recorded from few localities in Italy (both from the north and from the south), from Switzerland, from several Austrian provinces, from the Czech Republic and Slovakia, Hungary, Bosnia-Herzegovina, and from northwestern Turkey (material examined, BOHÁČ et al., 1993, HANSEN et al., 1995, Horion, 1967, KÖHLER \& KLAUSNitzer, 1998, Luigioni, 1929, MAjZLAN \& JÁSZAY, 1997, SCHEERPELTZ, 1968).

The ecologica data available are highly diverse and not very conclusive as regards the habitat requirements of the species. It has been observed in various types of woodland (beechwoods, mixed deciduous forests, xerothermous oakwoods), in grassland, arable land, and in bogs (material examined, HORION, 1967, KÖHLER, pers. comm.). On various occasions it has been found in or near old or rotting tree trunks inhabited by the ants Lasius fuliginosus and L. brunneus (GANGLBAUER, 1895, HORION, 1967). In the original description of A. glabriventris, RYE (1865) writes: " Several examples (accompanied by Homoeusa acuminata) were taken in May and June ... in the 'runs' leading from a nest ... of Formica fuliginosa, at the root of an old beech tree". One of the beetles from Schleswig-Holstein was taken from a rotting alder trunk; see also LOHSE (1967). LINKE (1962) found four specimens in black humus under grass roots. A considerable proportion of the specimens examined were collected by sweeping (May), at windows (July), and espe-

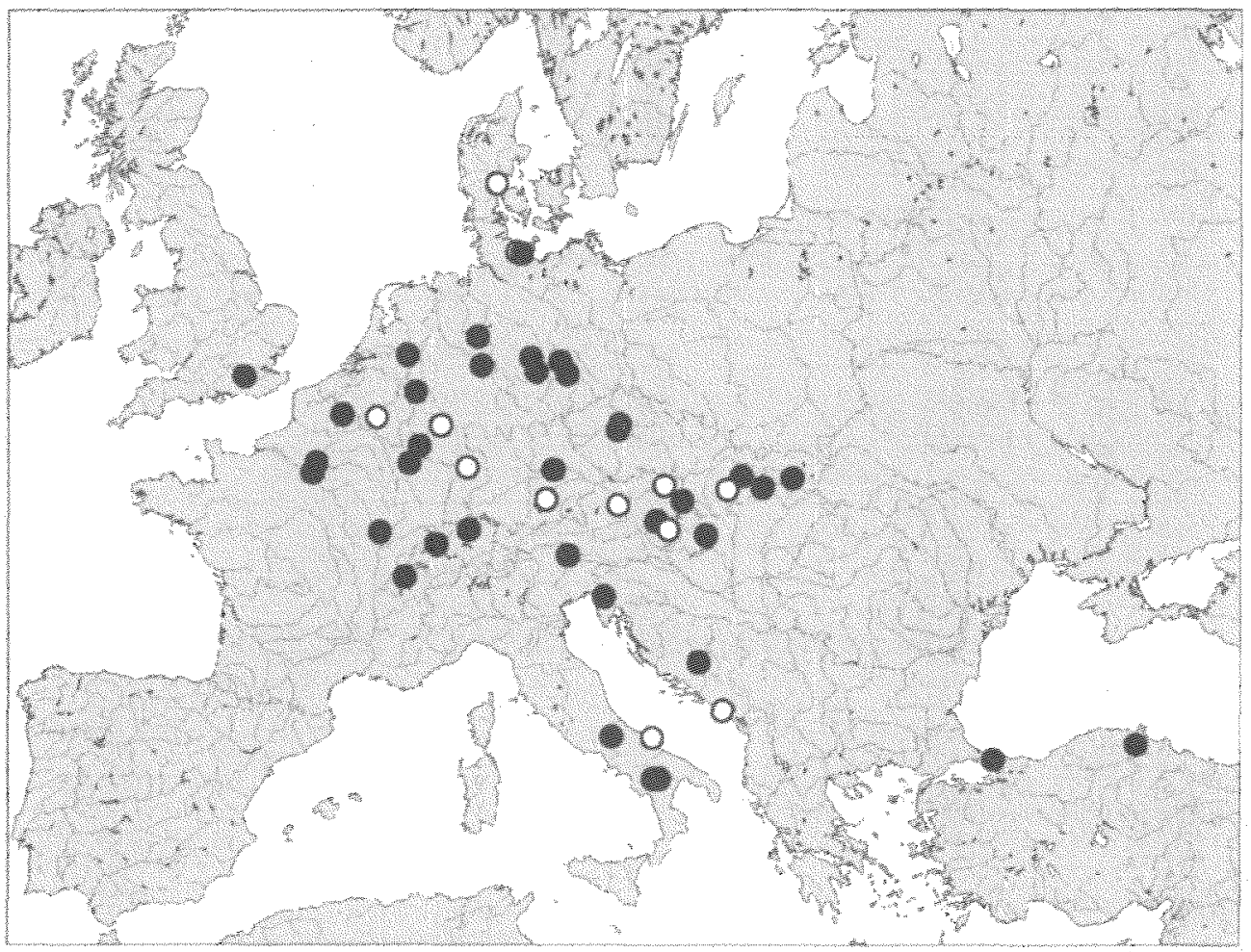

Map 3: Distribution of Amarochara bonnairei (FAUT'EL) based on revised records (filled circles) and selected literature records (open circles). 
cially in flight-intercept traps (May through July) (material examined and KÖHLER, pers. comm.). These observations suggest that $A$. bonnairei reproduces (and hibernates) in an unknown subterranean habitat and that it disperses by flight in late spring and early summer.

The vast majority of the material examined was collected during the period from May through July; only one specimen was found in August (Fig. 51). According to HORION (1967), the species has been found also April and September. Records from late autumn, winter, and early spring are unknown.

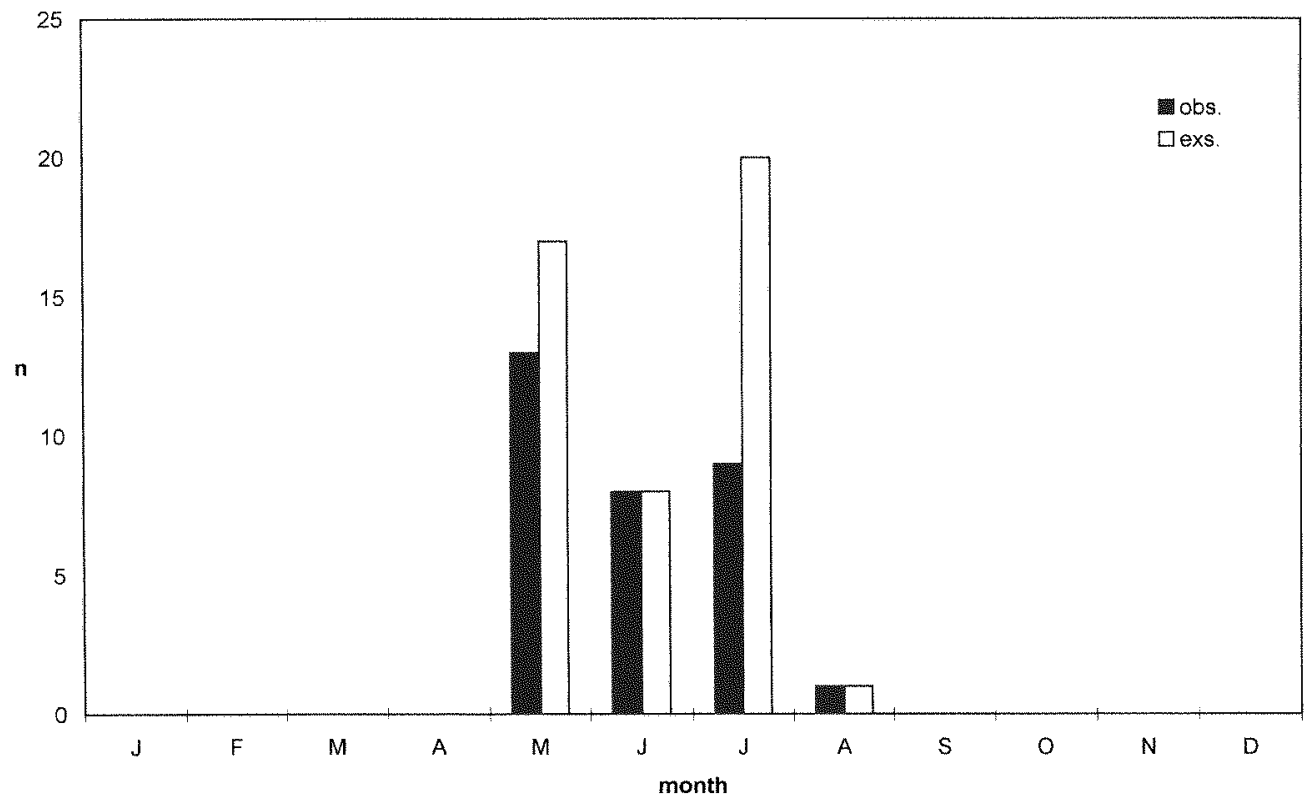

Fig. 51: Amarochara bonnairei (FAUVEL). Seasonal phenology based on material examined. Abbreviations: obs. $=$ number of observations or samples; exs. $=$ number of specimens.

\subsubsection{Amarochara cribripenmis (MULSANT \& REY, 1875)}

Figs. 52-57, Map 4

llyobates cribripennis MULSANT \& REY, 1875: 340f.

Amarochara (Lasiochara) cribripennis: BERNHAUER (1902).

\section{Types examined}

Lectotype $q$ [dissected prior to present study], here designated: $q$ / Corse Revel / Lectotypus Ityocara cribripennis M. R. des. J. Vogel 1991 / Amarochara bonnairei corr. J. Vogel / Lectotypus o Ihyobates cribripennis Mulsant \& Rey desig. V. Assing 2000/ Ama- 
rochara cribripennis (Mulsant \& Rey) det. V. Assing 2000 (MHNL). Paralectotype: 우 / Corse / Paralectotypus Ityocara cribripennis M. R. des. J. Vogel 1991 / Amarochara bonnairei cort. J. Vogel / Paralectotypus o Ilyobates cribripennis Mulsant \& Rey desig. V. Assing 2000/ Amarochara cribripennis (Mulsant \& Rey) det. V. Assing 2000 (MHNL).

\section{Comments}

The publication year indicated by most previous authors is 1874 (see e. g. BERNHAUER \& SCHEERPELTZ (1926)). According to BLACKWELDER (1952), however, the work containing the description of $A$. cribripennis was published in 1875 .

The original description is based on an unspecified number of syntypes from the "environs de Corte en Corse (collection Revélière)", two of which were located in the collections of the MHNL. J. Vogel, Görlitz, had examined these syntypes and attached a lectotype label to one of them, but a lectotype designation has not been published. In order to fix a single name-bearing type and preserve the present interpretation of the species, the $q$ in better condition is here designated as the lectotype.

\section{Additional material examined}

France: Corse: 2 exx., Pietranera, 1.V.1955, leg. Fagel (IRSNB, cAss); 1 q, Castagniccia, M. San Petrone, 1150m, sifted litter, 7.IV.1990, leg. Wunderle (cWun); 2 exx., Vizzavona, 11.VI.1909 (FIS); 1 ex., Bocognano, 900m, 9.V.1927 (FIS); 1 ot, Oletta, under stone, 27.IV.1973, leg: Vit (cAss); 1 ex., locality not specified, pitfall trap, 19.V.1969 (MHNG); 4 exx., locality not specified (DEI, MNHNP, NHMW).

Italy: Sardegna: 1 ex., 20km NW Nuoro, Lula, 15.V.1961 (MHNG); 10 , 2우, Bruncu Spina (NU), 1500m, 22.IV.1992, leg. Scheuern (cWun); 1 ex., Monti del Gennargentu, leg. Krüger (DEI); 1 ex., San Pietro island, Carloforte, 1918, leg. Dodero (SMTD); 1 ex., Ozieri, 20.II.1892, leg. Dodero (NHMW); 1 ex., Ozieri, 10.IV.1902, leg. Dodero (NHMW); 2 \% q, Ozieri, leg. Dodero (NHMW, cAss); 1 ex., Macomer (NHMB); 1 ex., locality not specified (NMP); 2 exx., locality not specified, U. Lostia (IRSNB).

\section{Redescription}

In size and many other external characters very similar to $A$. bonnairei. Colour of body variable; usual coloration: whole body brown to dark brown, with the hind margins of the abdominal segments and often the elytra and the antennae, at least partly, lighter brown; legs light brown; palpi testaceous. Occasionally, the body may be yellowish brown and of similar colour as in $A$. bonnairei, with only the head and the preapical abdominal segments somewhat darker. Microsculpture on head, pronotum, and abdomen absent or nearly so. Pubescence somewhat shorter and \pm decumbent, distinctly less erect than in A. bonnairei.

Head of \pm ovoid shape, indistinctly (approximately 1.05 times) wider than long, not distinctly dilated behind eyes; width across eyes equal to width across postgenae; eyes moderately convex, larger than in A. bonnairei, distinctly more than half the length of postgenae in dorsal view. Puncturation very fine and sparse, similar to that in $A$. bonnairei. Genal carina present. Antenna of similar morphology as in $A$. bonnairei, but longer and more massive (Fig. 57). Mouthparts as in A. bonnairei.

Pronotum of similar shape as in $A$. bonnairei, but on average broader, approximately 1.10 - 1.15 times as wide as long and 1.25 - 1.35 times as wide as head; maximal width in or near the middle; puncturation very fine, finer than in average $A$. bonnairei, and illdefined. 


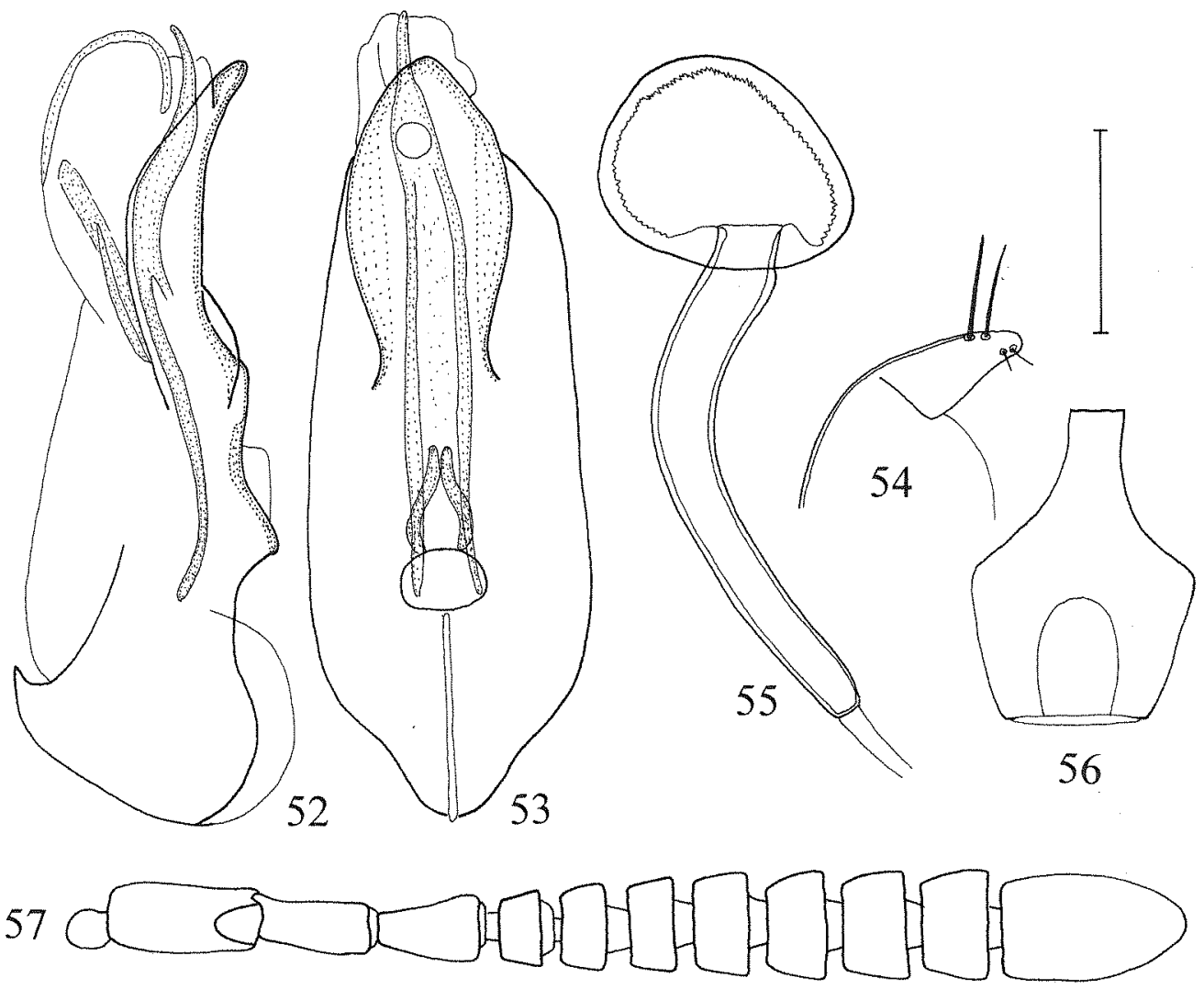

Figs. 52-57: Amarocbara cribripennis (MULSANT \& REY). Median lobe of aedeagus in lateral and in ventral view $(\mathbf{5 2}, \mathbf{5 3})$; apical lobe of paramere (54); spermatheca (55); tergum $X$, pubescence omitted (56); antenna, pubescence omitted (57). Scale: 54-55: 0.1 mm; 52-53:0.125 mm; 56-57:0.2 mm.

Elytra on average relatively longer than in $A$. bonnairei, at suture $0.8-0.9$ times the length of pronotum and usually $1.10-1.15$ times as wide as pronotum; posterior margin near posterior angles weakly sinuate; puncturation distinctly denser and more granulose than in A. bonnairei. Hind wings fully developed. Legs long and slender, first metatarsomere very long, longer than in average $A$. bonnairei, at least as long as the three following tarsomeres. Mesosternum with microsculpture similar to that in $A$. umbrosa, without median carina; mesosternal process apically acute.

Abdominal terga III-V with moderately deep, tergum VI without appreciable anterior impression; sterna III-V anteriorly constricted; puncturation as in $A$. bonnairei sparser in posterior than in anterior parts of terga and sparser on posterior than on anterior terga, but on the whole much denser and more distinct, particularly so on terga III and IV; tergum $\mathrm{X}$ with long anterior extension (Fig. 56).

$\sigma^{*}$ : tergum and sternum VIII as in $A$. bonnairei, but marginal setae of sternum VIII longer; aedeagus of similar general morphology as in $A$. bonnairei, but ventral process of median lobe in ventral view broader, in lateral view subapically more distinctly curved, 
and with more distinct basal folds (Fig. 52-53); apical lobe of paramere similar to that in A. bonnairei (Fig. 54).

i: tergumVIII, sternum VIII, and spermatheca (Fig. 55) similar to those in A. bonnairei.

\section{Comparative and systematic notes}

From the similar A. bonnairei, A. cribripennis is readily distinguished above all by the (mostly!) darker colour, the absence of microsculpture on head and pronotum, the longer and more massive antennae, the denser and coarser puncturation of the elytra and the abdomen (especially on terga III and IV), and by the morphology of the aedeagus. For distinction from other West Palaearctic congeners see the species sections below and the key in section 5 .

A. cribripennis is apparently the adelphotaxon of $A$. bonnairei. Both species are highly similar in external and sexual characters and share the following synopomorphies: abdominal terga III-VI anteriorly with fine puncturation, ventral process of aedeagus basally with lateral carinae, and internal tube of aedeagus with very long basal part (see section 3.3).

\section{Distribution and bionomics}

A. cribripennis has a Tyrthenian distribution sensu LATTIN (1967); its range is confined to Corsica and Sardinia (Map 4). It has been found by sifting leaf litter, under stones, and with pitfall traps at elevations of $900-1500 \mathrm{~m}$ in February and during the period from April through June (material examined).

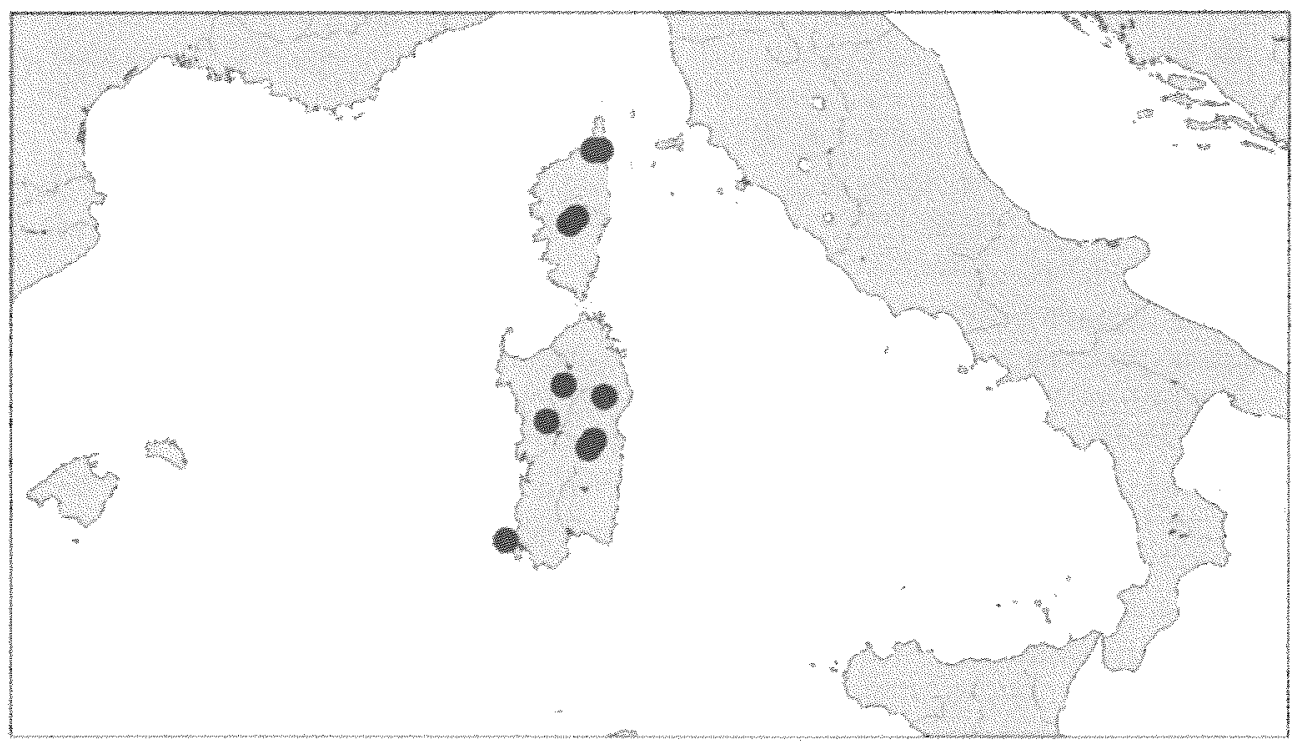

Map 4: Distribution of Amarochara cribripennis (MULSANT \& REY) in Corsica and Sardinia based on tevised records. 


\subsubsection{Amarochara siculifera sp. $\mathrm{n}$.}

Figs. 58-61, Map 5

\section{Types}

Holotype ơ: Namrun, Anat.m., 11.-26.5.60, leg. F. Schubert / Holotypus o Amarochara siculifera sp. n. det. V. Assing 2000 (NHMW).

Paratypes: 1 9 : 831 / 246 / versicolor Fauv. Syria. Beirut. Mus. Vindob. / c. Epplsh. Steind. d. (NHMW); $10^{\star}: 832$ / B. M / versicolor Fauv. Libanon. Mus. Vindob. / crassicornis Quedenf. Berl. Zt. 1882 p. 181 / c. Epplsh. Steind. d. (cAss); 1 o : o [sic] / Libanon, Dr. Couteau [?] / Amarochara crassicornis Quedenf. / ex coll. Scheerpeltz (NHMW); 1 q: Turquie Mersin S/ Yeniköy, 1000-1200m, 29.IV.78, Besuchet Löbl (MHNG).

\section{Description}

Relatively large species, $3.8-4.6 \mathrm{~mm}$ (abdomen fully extended). Colour of body variable: forebody uniformly rufous or bicoloured; head and pronotum rufous to dark brown, elytra rufous to light brown; abdomen dark brown to blackish, with the tergal margins and the abdominal apex lighter; legs and antennae testaceous to light brown; mouthparts testaceous. Forebody and most of abdomen without appreciable microsculpture, apical abdominal segments occasionally with traces of shallow transverse microsculpture. Pubescence of forebody relatively long and suberect to decumbent.

Head of subcircular shape, approximately as wide as long; not or only weakly dilated behind eyes; eyes moderately large, weakly convex, not distinctly projecting from lateral outline of head, distinctly more than half the length of postgenae in dorsal view, the latter smoothly curved towards posterior constriction. Puncturation sparse and extremely fine. Genal carina present, but fine. Antenna very long and massive, similar to that in the following species (cf. Fig. 66), antennomere I with dorso-apical furrow reduced, very short and inconspicuous; antennomere III as long as II. Third joint of maxillary palpus very long and slender (Fig. 61), similar to that in A. bonnairei.

Pronotum large in relation to head, 1.15 - 1.25 times as wide as long and approximately 1.4 times as wide as head or even wider; maximal width in or near the middle; posterior angles \pm ill-defined; puncturation similar to that of head.

Elytra of similar relative length as in $A$. bonnairei, at suture usually $0.75-0.80$ times the length of pronotum and approximately $1.15-1.20$ times as wide as pronotum; posterior margin near posterior angles noticeably sinuate, somewhat more so than in $A$. bonnairei (but distinctly less than in $A$. forticornis); puncturation weakly granulose, more distinct than in $A$. bonnairei and much more conspicuous than that of pronotum. Hind wings fully developed. Legs long and slender, first metatarsomere approximately as long as the combined length of the three following tarsomeres or slightly longer.

Abdominal terga III-V with deeper anterior impressions than in $A$. bonnairei, tergum VI without or with very weak anterior impression; sterna III-V anteriorly constricted; puncturation in impressions of terga III-V and near anterior margin of tergum VI very coarse and dense, on remainder of dorsal abdominal surface distinctly finer and sparser, punctures on abdomen on the whole coarser, deeper and larger than in $A$. bonnairei.

$\sigma^{*}$ : posterior margin of tergum VIII moderately convex, with a row of moderately long 
and a sparse row of long thin setae; posterior margin of sternum VIII strongly convex, with a dense row of relatively long marginal and a sparse row of very long submarginal thin setae; aedeagus of similar general morphology as in A. bonnairei, with ventral process distinctly separated from median lobe; at base of ventral process with remarkable median process (similar to the process of the crista apicalis in species of Geostiba THOMSON s. str.), crista apicalis relatively small, crista proximalis pronounced; internal sac with long tube (Figs. 58-59).

q: tergum VIII of similar shape and chaetotaxy as in $\sigma^{*}$, but with denser marginal setae; posterior margin of sternum VIII weakly convex, row of marginal setae very weakly modified, relatively thin, and short; spermatheca as in Fig. 60.

Derivatio nominis: The name (Lat., adj.: siculus = small dagget) refers to the distinctive median process at the base of the ventral process of the aedeagus.

\section{Comparative notes and comments}

From A. bonnairei and A. cribripennis, this species is separated by larger size, a distinctly larger and more transverse pronotum (especially in relation to head), by more massive and longer antennae, by the much coarser puncturation of the anterior impressions of the abdominal terga III-V, and by the morphology of the aedeagus. A siculifera is extremely similar to the following species, from which it is distinguished with absolute certainty only by the shape of the aedeagus. Due to relatively high intraspecific variation in external characters and some overlap in the distinguishing characters indicated in the description of $A$. inermis sp. n., it may be difficult to attribute the $q 9$ to either of the species.

\section{Distribution and bionomics}

The known distribution of the species, evidently a Syrian element sensu LATTIN (1967), ranges from central southern Anatolia to Lebanon (Map 5). Two of the types were collected in April and May. Apart from the altitude (1000-1200m) indicated on the label attached to the specimen from Mersin, no further bionomic data are available.

\subsubsection{Amarachara inermis sp. n.}

Figs. 62-67, Map 5

\section{Types}

Holotype o : ISRAËL: côte Maagan Mikhael, 16.IV.1982, Besuchet Löbl / Holotypus ơ Amarochara inermis sp. n. det. V. Assing 2000 (MHNG).

Paratypes: $10^{*}$ : ISRAËL: Galilée, Eilon, N. Betzet, 20.IV.1982, Besuchet Löbl (MHNG); $2 \sigma^{0}, 2$ ㅇ ㅇ: ISRAËL: Galilée, Ginosar; -200m, 28.IV.1982, Besuchet Löbl (MHNG, NHMW, cAss); 1 ex.: ISRAËL: Golan, Mahjar; -200m, 27.IV.1982, Besuchet Löbl

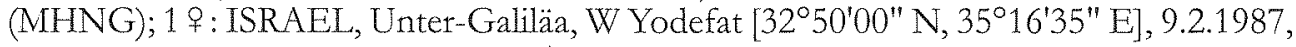
$300 \mathrm{~m}$, leg. Schawaller \& Schaufuss / Amarochara crassicornis Quens. [sic] det. E. Ulbrich 1988 (SMNS); $10^{*}$ : Bonnairei Fauv. Gal. / coll. Hampe (cAss). 


\section{Description}

Externally almost identical to $A$. siculifera, distinguished only by the following characters: Body on average more distinctly bicoloured, with pronotum and elytra yellowish brown to rufous and with head and most of abdomen dark brown to blackish. Antenna in most specimens with less transverse antennomeres $\mathrm{III}-\mathrm{V}$ and with antennomeres IV and V less distinctly differing in length (Fig. 66).

First metatarsomere longer than in average $A$. siculifera, usually longer than the combined length of the three following metatarsomeres (Fig. 67).

0 : aedeagus with ventral process longer and less distinctly tapering apically, at base without long process (Figs. 62-63); apical lobe of paramere as in A. siculifera (Fig. 64). ㅇ: shape of spermatheca very similar to that of $A$. siculifera (Fig. 65).

\section{Derivatio nominis}

The name (Lat., adj:: unarmed) refers to the fact that, in contrast to the otherwise extremely similar $A$. siculifera, the aedeagus of $A$. inermis lacks the conspicuous median process at the base of the ventral process.

\section{Comparative and systematic notes}

For distinction from $A$. siculifera, evidently the sister species of $A$. inermis, the description above is referred to. For separation from other species of Amarochara see the comparative notes below $A$. siculifera.

\section{Distribution and bionomics}

The species is known only from Israel, where the types were collected in February and April at low elevations of up to $300 \mathrm{~m}$ (Map 5). The specimen from Yodefat was collected in Quercus macchia (SCHAWALLER, pers. comm.).

\subsubsection{Amarochara caeca sp. n.}

Fig. 68

\section{Types}

Holotype 우 18.3.84 Tunesien, Umg. Ain Draham, leg. H. Meybohm / Holotypus of Amarochara caeca sp. n. det. V. Assing 2000 (cAss).

Paratype o : T. [ETunisia] Le Kef, 3-1940, Dr. Normand / B. 6811 / Paratypus 9 Amarochara caeca sp. n. det. V. Assing 2001 (c'Tro).

\section{Description}

Length of holotype: $4.0 \mathrm{~mm}$ (abdomen fully extended). Colour of head, pronotum, elytra, and abdomen (except for the slightly lighter tergal hind margins) uniformly blackish; legs pitchy brown; antenna brown, with the antennomeres I-III infuscated; maxillary palpi testaceous. Head and pronotum with shallow, but distinct microsculp- 

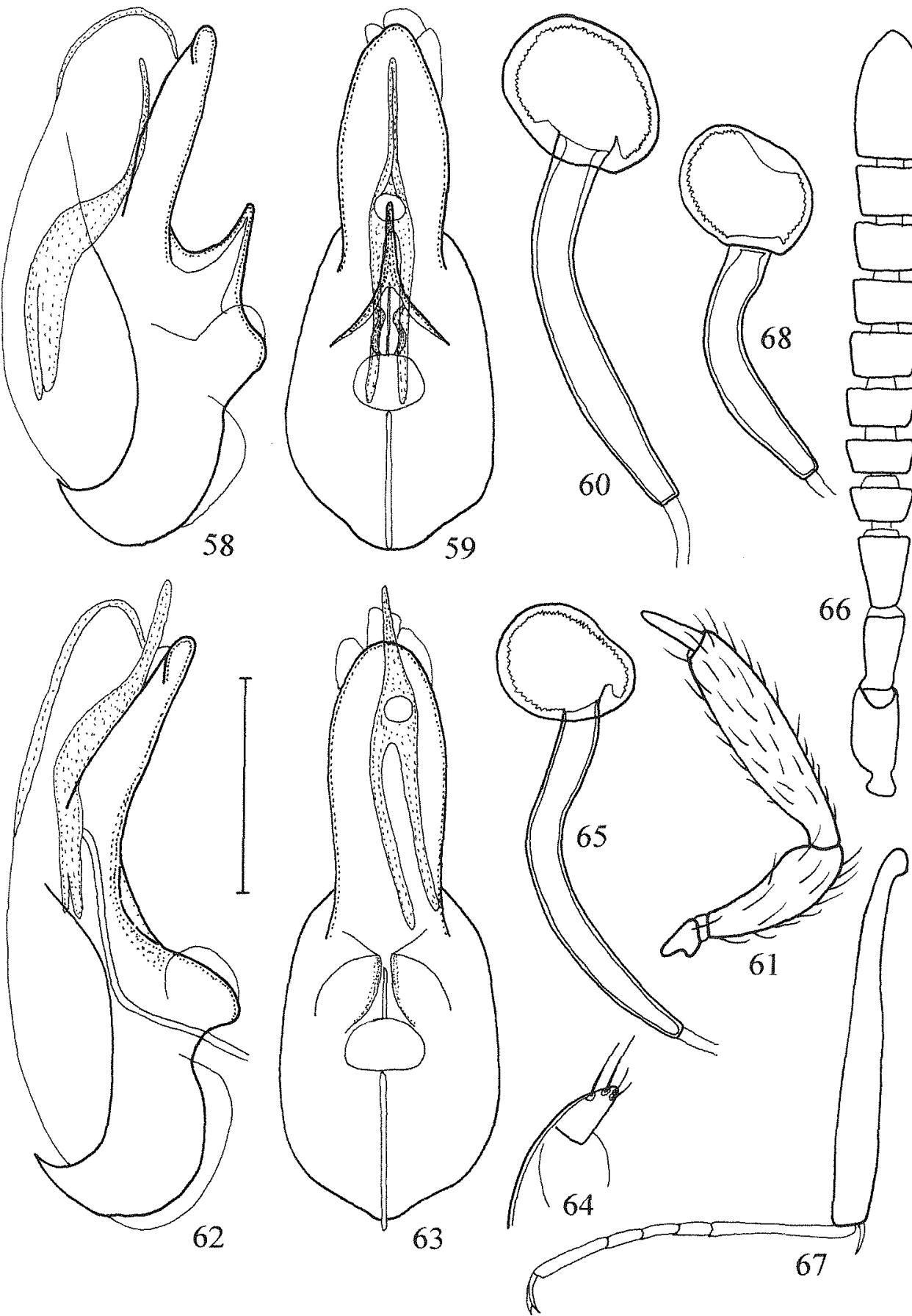

Figs. 58-68: Amarochara siculifera sp. n. (58-61), A. inernis sp. n. (62-67), and A. caeca sp. n. (68). Median lobe of aedeagus in lateral and in ventral view $(58,59,62,63)$; spermatheca $(60,65,68)$; maxillary palpus $(61)$; apical lobe of paramere (64); antenna, pubescence omitted (66); metatibia and metatarsus, pubescence omitted (67). Scale: 58-65: 0.2 mm; 66-67:0.4 mm. 
ture (similar to $A$. bonnairez), elytra only near hind margin with traces of transverse microsculpture; abdominal terga III-VI without, tergum VII with very fine transverse microsculpture. Pubescence of forebody moderately long and suberect, similar to that in $A$. bonnairei.

Head of subcircular shape, indistinctly wider than long; not dilated behind eyes; eyes large (much larger than in $A$. bonnaire 2 ), moderately convex, slightly projecting from lateral outline of head (maximal width across eyes), almost as long as postgenae in dorsal view, the latter smoothly curved towatds posterior constriction. Puncturation sparse and extremely fine. Genal carina present, but fine. Antenna longer and more massive than in $A$. bonnairei; antennomere III as long as II, IV and V short and strongly transverse, IV - $\mathrm{X}$ not gradually increasing in length (as is the case in $A$. bonnairel), but $\mathrm{V}$ distinctly (approximately 1.5 times) longer and less transverse than $\mathrm{V}$, and $\mathrm{V}-\mathrm{X}$ almost subequal in length; antennomere I with dorso-apical furrow reduced, very short and inconspicuous. Third joint of maxillary palpus very long and slender, similar to that in $A$. bonnairei.

Pronotum larger in relation to head than in $A$. bonnairei, 1.14 times as wide as long and approximately 1.3 times as wide as head; maximal width approximately in the middle; posterior angles weakly marked; puncturation very fine and sparse, barely noticeable in the microsculpture.

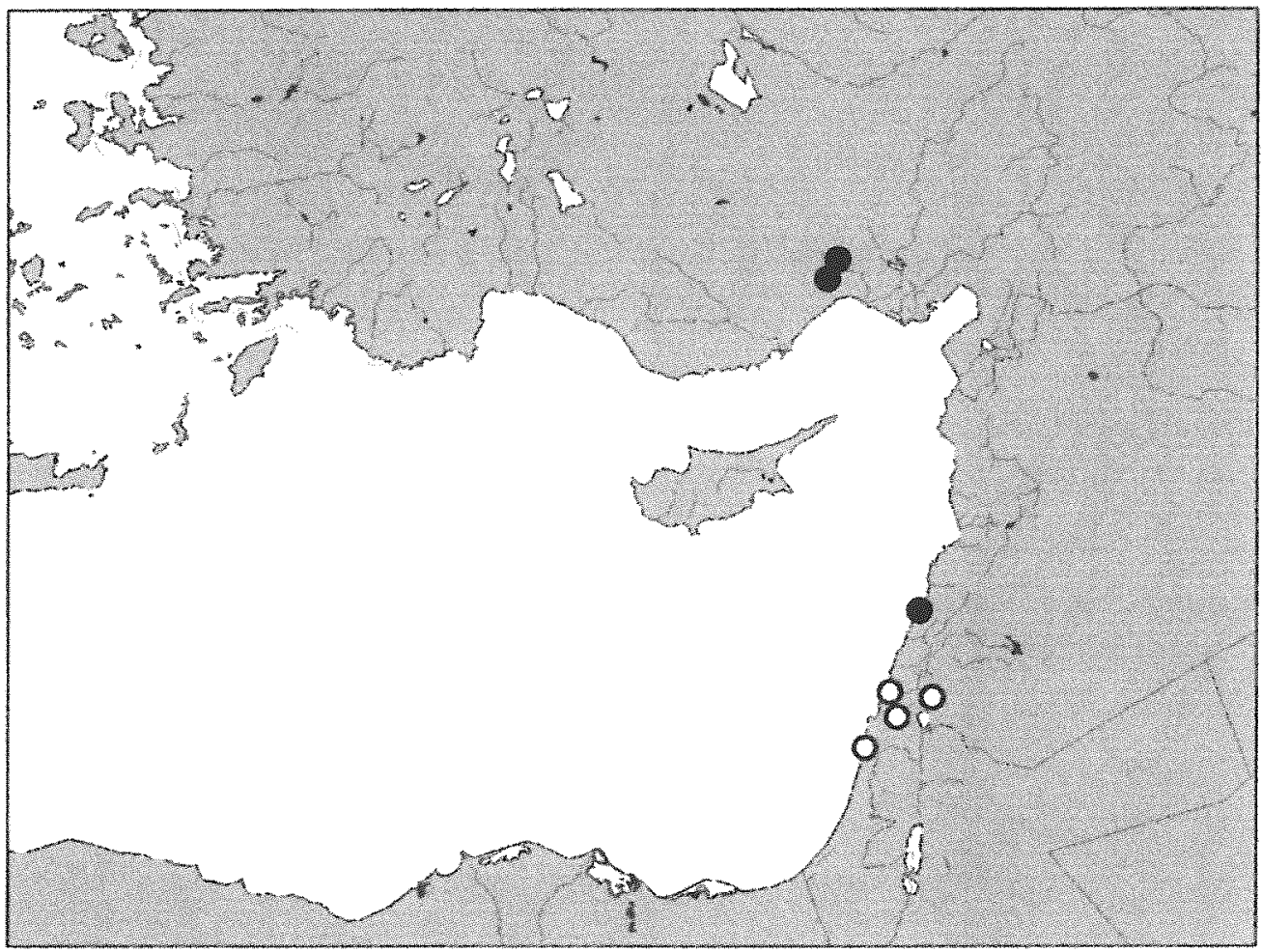

Map 5: Distribution of Amarochara siculifera sp. n. (filled circles) and A. inermis sp. n. (open circles) in the Eastern Mediterranean. 
Elytra relatively longer than in $A$. bonnairei, at suture usually 0.84 times the length of pronotum and 1.17 times as wide as pronotum; posterior margin near posterior angles as in $A$. bonnairei weakly sinuate; puncturation indistinctly granulose, somewhat denser and slightly coarser than in A. bonnairei, and much more conspicuous than that of pronotum. Hind wings fully developed. Legs long and slender, first metatarsomere approximately as long as the combined length of the three following tarsomeres.

Abdominal terga III-V with impressions of similar depth as in $A$. bonnairei, tergum VI without anterior impression; sterna III-V anteriorly constricted; puncturation in impressions of terga III-V and near anterior margin of tergum VI rather coarse and dense, much coarser and denser than in $A$. bonnairei, on remainder of dorsal abdominal surface distinctly finer and sparser, but slightly more conspicuous than in $A$. bonnairei.

ot: unknown.

O: tergum VIII posteriorly moderately convex and with row of rather long thin marginal setae; posterior margin of sternum VIII weakly convex, row of marginal setae almost unmodified, relatively thin, and moderately short; spermatheca as in Fig. 68.

Derivatio nominis: The name (Lat., adj.: dark) refers to the distinctive uniformly dark colour of the species.

\section{Comparative notes and comments}

From other Western Palaearctic congeners, $A$. caeca is distinguished especially by its uniformly dark colour and by the distinct microsculpture of the head and pronotum, which it only shares with $A$. bonnairei. For additional characters separating it from the latter species and from the similarly dark $A$. splendens see description above and section 4.6 , respectively.

\section{Distribution and bionomics}

The species is currently known only from Tunisia. Data other than those indicated on the labels attached to the two type specimens are not available.

\subsubsection{Amarochara crassicornis (QUEDENFELDT, 1882)}

Figs. 69-70

Ilyobates crassicornis QUEDENFELDT, 1882: 181.

Amarochara (Lasiochara) crassicornis: BERNHAUER. (1902).

\section{Type examined}

Holotype [severely damaged: part of the legs, abdominal segments VI and following missing]: Maroc / crassicornis / 6718 / Type / Ilyobates crassicornis Quedf. / Amarochara/ Holotypus Ilyobates crassicornis Quedenfeldt, rev. Assing 1998 (MNHUB). 


\section{Redescription}

Of similar size as $A$. forticornis (see section 4.3.1). Head dark brown, pronotum brown, elytra yellowish brown; abdomen dark brown with lighter tergal margins; antennae brown, with slightly lighter basal antennomeres; legs and mouthparts testaceous. Head and pronotum with very shallow, but noticeable microsculpture. Pubescence of forebody similar to that in $A$. forticornis.

Head of subcircular shape, indistinctly transverse; eyes large, though not strongly convex, slightly more distinctly projecting from lateral outline of head than in $A$. forticornis, their diameter almost as long as postgenae in dorsal view. Puncturation finer, shallower, and less distinct than in A. forticornis. Genal carina present. Antenna long and massive, of similar morphology as in A. forticornis, but antennomeres IV and V less transverse (Fig. 69). Mouthparts similar to those in A. forticornis.

Pronotum relatively large and broad (width: $0.74 \mathrm{~mm}$ ), 1.18 times as wide as long and 1.4 times as wide as head; maximal width approximately in the middle; posterior angles weakly marked; puncturation similar to that of head, but denser.

Elytra relatively longer and broader than in A. forticornis; at suture 0.85 times the length of pronotum and approximately 1.1 times as wide as pronotum; posterior margin near posterior angles weakly sinuate; puncturation much finer than in $A$. forticornis and not granulose. Legs very long and slender; length of metatibia and metatarsus (without claws) $0.72 \mathrm{~mm}$ and $0.63 \mathrm{~mm}$, respectively; first metatarsomere slightly longer than the combined length of the three following tarsomeres (Fig. 70). Mesosternum without median carina; mesosternal process apically rounded, almost acute.

Abdominal terga III- $V$ with anterior impressions of similar depth as in $A$. carinata (see section 4.3.2), distinctly less deep than in $A$. forticornis; puncturation of terga III-V similar to that in $A$. carinata (much denser than in $A$. forticornis), only slightly coarser and denser in anterior impressions than on remainder of dorsal surface. Sexual characters missing in the holotype.

\section{Comparative notes}

From $A$. forticornis, the species is readily distinguished especially by the larger eyes, the larger and broader pronotum, the presence of shallow microsculpture on head and pronotum, the weakly sinuate posterior margins of the elytra, the much finer elytral puncturation, the longer legs, the shallower transverse impressions on the abdominal terga III$\mathrm{V}$, and the puncturation of the abdomen, which is finer in the anterior impressions of terga III-V and much denser on the posterior parts of these terga. A. carinata, which is similar in coloration and many other external characters, differs from $A$. crassicornis in the morphology of the antennae (shorter antennomere III, much more transverse antennomeres IV-X, antennomere $\mathrm{V}$ approximately 3 times as wide as long), in the much smaller eyes, the absence of microsculpture on head and pronotum, the smaller and less transverse pronotum, the relatively shorter elytra, the distinctly shorter legs, the presence of a carina on the mesosternum, and the slightly less dense abdominal puncturation. 


\section{Distribution and bionomics}

A. crassicornis has become known only from Morocco, Algeria, and Tunisia (COIFFAIT, 1973, NORMAND, 1934, QUEDENFELDT, 1882). Literature records from the eastern Mediterranean refer to either A. inermis or A. siculifera. The record from Sicily (PORTA, 1926, ZANETTI, 1995) should be considered doubtful until the corresponding specimen has been revised. Neither the original description nor the labels attached to the holotype reveal any data regarding the circumstances of collection.

\subsection{The forticornis group}

Primarily based on the presence of a median carina on the mesosternum, two species are here tentatively attributed to this group. However, as is the case with the bonnairei group, the monophyly of $A$. forticornis and $A$. carinata is only weakly supported, especially because the remaining synapomorphies also occur in other Amarochara and because the male sexual characters of $A$. carinata as well as of some species of the bonnairei group are unknown (for further details see section 3.3). In addition, A. forticornis is characterized by several distinct autapomorphies, especially the presence of a distinct anterior impression on abdominal tergum VI and the posteriorly strongly sinuate elytra.

\subsubsection{Amarochara forticornis (LACORDAIRE, 1835)}

Figs. 71-77, 80-81, Map 6

Bolitochara forticornis LACORDATRE, 1835: 543.

Calodera forticornis: ERTCHSON (1839).

Ibrobates forticornis: KRAATZ (1856).

Iyobates (Mniobates) forticomis: MULSANT \& REY (1875).

Amarocbara (Mniobates) forticornis: GANGLBAUER (1895).

\section{Material examined}

Great Britain: $1 \delta^{7}$, Hampshire, Titchfield near Gosport, Upper Titchfield Haven, flood litter, 24.I.1971, leg. Appleton (cOwe).

Spain: 297 , "Hispania" (SMTD, cAss).

France: Bretagne: 1 ex., Lorient (NHMW). Aquitaine: 1 ex., Camblanes, 15.IV.1902 (MHNG). Centre: 1 ex., Perrusson, X.1905, leg. Méquignon (MHNG); 2 exx., Perrusson, inundation, leg. Méquignon (MHNG); 2 exx., Vendôme (MHNG); 6 exx., Loire-et-Cher, Meslay, I.1910, leg. Méquignon (MHNG). Île-de-France: 1 ex., Meaux, I.1910 (NHNG); *3 exx., Bray/Seine, flood refuse, 25.I.1981, leg. Tronquet (cTro); 1 ex., Paris, leg. Fischer (MNHUB); 2 exx., Paris (DEI). Champagne-Ardennes: 1 ex., Corbeil, 28.V.1915, leg. Méquignon (MHNG). Franche-Comté: 2 ex., Fraisans, 30.X.1935 \& 9.III.1939, leg. Gruardet (MHNG, cAss). Rhône-Alpes: 1 ex., Lyon, leg. Fischer (MNHUB); 1 ex., Jardin (MHNG); 1 ex., Pierre-Bénite, inundation (MHNG). Midi-Pyrénées: 2 exx., Toulouse (MHNG, SMNS). Languedoc-Roussillon: 10 , Hérault, Lattes, leg. Perrot (cAss); 1 ex., *Molitg-les-Bains, soil washing, 20.XII.1996, leg. Tronquet (cTro). Provence: 1 ex., Var, Argens inundation, III.1956 (MHNG); 1 ex., Var, Marais inundation, XII.1959 (MHNG); 4 exx., Var, Fréjus, Marais inundation, XII.1956 (MHNG); $10^{\circ}$. Fréjus, XII.50; 1 ex., Siagne (MHNG); 1 ex., Siagne inundation, XI.1951 (MHNG); $10^{3}$, Loup inundation, II.1951 (cAss); $10^{3}$, Loup inundation, XI.1951 (cAss); 2 exx., Nice (MHNG); 1 \&, Camargue, Bouches-du-Rhône, XI.1979 (MHNG). 
Alsace: 2 exx., Haguenau ["Hagenauer Forst"] (MNHUB) Locality not specified, not identified, or ambiguous: 1 ex., Gazinet, 27.IV.1901 (MHNG); 1 ex., Casaux, 22.V.1904; 1 ex., Mt. Lembert, 2.XI.1918 (MHNG); 6 exx., "Gallia" (DEI, FIS, MHNG, SNTD); 1 ex., "Gallia mer.", leg. Reitter (NMP); 1 ex., "Gallia m." (NMP).

Germany: Schleswig-Holstein: 1 ex., Husum, leg. Varendorff (NHMW). Niedersachsen: $30^{\circ}, 1$, Hannover, Langenhagen, ditch, pitfall, 17.-31.V.1991, leg. Sprick (cAss); $30^{*} \sigma^{*}, 2 q q$, same data, but 1.30.VI.1991 (cAss, cWun); $20^{*}$, same data, but 30.VI.-20.VII.1991 (cAss); 1 ex., Hannover, Ricklinger Teiche, 22.IV.1983, leg. Assing (cAss); 1 \%, Hannover, Herrenhausen, bank of Leine river, pitfall, 14.IV.1990, leg. Assing (cAss); 10 , same data, but 4.IV.1990 (cAss); 2 우, same data, but 15.V.1990 (cAss); 3 우 옹 same data, but 7.VI.1990 (cAss); 1 \%, 1 ex., Hannover, Stöcken, fallow, pitfall, 3.V.1986, leg. Assing (cAss); $10^{*}$, same data, but 16.VI.1986 (cAss); 1 ex., same data, but 2.VIII.1986 (MHMG); 1 ex., same data, but 18.VIII.1986 (cWun); $10^{7}$, same data, but 7.V.1987 (cWun); 10, Hannover, Stöcken, bank of Leine river, flood debris, 31.I.1994, leg. Assing (cAss); 2 q $q$, Hannover, Ahlem, fallow, pitfall, 13.V.1992, leg. Sprick (cAss); 1 ex., Hannover, garden, pitfall, 3.V.1986, leg. Assing (cKöh); 1 9, same data, but 17.V.1986 (cAss); 10 , S Hildesheim, Steinberg, Mesobrometum, pitfall, 1.IV.1997, leg. Schmidt \& Sprick (cAss); 1 \%, same data, but 1.V.1997 (cAss); $20^{\star}$ o $^{\star}$, same data, but 17.V.1997 (cAss); $10^{\star}, 1$ 9, $3 \mathrm{~km} \mathrm{NE} \mathrm{Alfeld/L.,} \mathrm{Wernershö-}$ he, arable land, pitfall, V.1998, leg. Schmidt \& Sprick (cAss); 1 \%, same data, but III.1998 (cAss); 1 \%, same data, but TV.1998 (cAss); 1 ㅇ, same data, but VI.1998 (cAss); 1 ex., Alfeld/Leine, Ortsberg, Mesobrometum, pitfall, 31.VII.1985, leg. Assing (cAss); 19 , Osnabrück, Hasetorwall, pitfall, VIII.1993, leg. Kache (cAss). Nordthein-Westfalen: 1 \%, Zülpich, 12.IX.1978, leg. Boneß (cRen); 5 exx., Wesseling, Rheinaue Urfeld, 30.I.1995, leg. Köhler (cKöh, cKap); 1 ex., Niederkassel, Rheinaue Rheidt, 24.I.1995, leg. Köhler (cKöh); 2 exx., Erftstadt, Kiesgrube Herrig, 27.VII.1994, leg. Köhler (cKöh); 1 ex., Bornheim, Hemmerich, garden, IV.1995, leg. Köhler (cKöh); 1 우, Kreis Unna, Geisecke, pitfall, 12.-26.VI.1996, leg. Flake (cFel); 1 ex., Elberfeld, leg. Cornelius (NHMW); 1 ex., Kreis Coesfeld, E Billerbeck, Baumberge, 51 58N, 07²3E, deciduous forest, malaise trap, VIII.1998, leg. Ribbrock (cFel); 1 ex., Jülich, leg. Eichhoff (NHMW); 1 ex., Krefeld (DEI). Rheinland-Pfalz: 2 exx., Nahetal, Aue Staudernheim, 24.I.1995, leg. Köhler (cKöh); 4 exx., Mannheim, 15.V.1917 (NHMB, SMNS); 10, 3 exx., Ingelheim, Neumühle, arable land, 1993, leg. Köhler (cKöh, cAss); 3 exx., Speyer, XI.1882 (NHMW); 1 ex., Speyer (MNHUB); 1 ex., Dürkheim (NHMW); 15 exx. [1 ex. with worker of Lasius platythorax attached to the pin], Ludwigshafen, 15.V.1917, leg. Ihssen (MNHUB). Baden-Württemberg: 1 ex., Dürrheim, leg. Eppelsheim (DEI); 1 ex., Freiburg (MNHUB); 2 exx., Neckarsulm, 22.IV.1957, leg. Ullrich (SMNS); 1 ex., Gottenheim, 10.VI.1980, leg. Lunau (SMNS); 1 ex., "Breisgau" (MNHUB); 1 ex., Schwarzwald (NHNWW); 1 ex., Baden, locality illegible (MNHUB), Bayern: 1 ex., Plattling, Isar-Auen, 16.V.1920, leg. Stöcklein (NHMB); 1 ex., Hainsbach, 3.V.1924, leg. Stöcklein (NHMB); 1 ex., Mitterteich, car-net, 20.V.1998, leg. Köhler (cKöh). Hessen: 1 ex., Höchst, 22.III.1914, leg. Sattler (FIS); 1 ex., Darmstadt (DEI). Mecklenburg-Vorpommern: 2 exx., "Mecklenburg" (NHMW). Sachsen-Anhalt: 1 ex., Eisleben (FIS); 1 ex., Athenstedt near Halberstadt, 25.X.1981, leg. Jung (cVog); $10^{*}$, Merseburg, 9.IV.1982, leg. Fritsche (cVog); 4 exx., Naumburg, leg. Maertens (SMTD); 12 exx., Naumburg, 12.-26.V.1934, leg. Maertens (MNHUB, NHMB). Brandenburg/Berlin: 14 exx., Kalkberge near Rüdersdorf, leg. Neresheimer (DEI); 1 ex., Liepnitzsee (MNHUB). Thüringen: 1 ex., Seehausen, Seehäuser Ried, pitfall, 1.V.1996, leg. Grimm (NME). Sachsen: 1 ex., Leipzig, Lindenthal, 19.IV.1914, leg. Linke (SMTD); 1 ex., Leipzig, Seifertshain, 8.XI.1977, leg. Linke (SMTD). Locality not specified: 1 ex., "Rheinland" (DEI).

Switzerland: Basel: 1\%, Pratteln, fallow "Löli", pitfall, 10.-17.V.1997, leg. Luka (cFel); 2 exx., Allschwil, 17.IV.1941 (MHNG).

Austria: Niederösterreich/Wien: 1 ex., Ulrichskirchen, leg. Spurny (FIS); 1 ex., Ulrichskirchen (DEI); 3 exx., Donauauen, leg. Breit (FIS, NHMB, NHMW); 2 exx., Stockerau, leg. Bernhauer (NHMN, SMINS); 1 ex., Korneuburg, leg. Liepoldt (NHMB); 1 ex., Moosbrunn, 6.IV.1953, leg. Schubert (NHMW); 1 ex., Herzogenburg, leg. Grundmann (NHMW); 1 ex., Weidlingburg (NHMW); 1 ex., Kahlenberg, leg. Blühweiss (NHMW); 1 ex. [with worker of Lasius brunneus attached to the pin], Lg.-Enzersdorf, leg. Luze (NHMW); 1 ex. [with worker of Lasius brunneus attached to the pin], Bisamberg, leg. Luze (NHMW); 2 exx., Vöslau (NHMB); 1 ex., Wien, Strebersdorf, 21.IV.1901 (NHMW); 1 ex., Albern near Wien, Donauauen, leg. Scheerpeltz (NHMW); 2 exx., Wien Kagran, leg. Kniz (NMP); 2 exx., Wien, Laaerberg (NHMW); 8 exx., Wien env., leg. Winkler, Haberditz, Strauss (NHMB, NHMW, SMTD); 1 ex., Wienerwald (NHMW); 1 ex., Marchfeld, Oberweiden (MNHUB); 1 ex., locality not specified, leg. Zoufal (MHNG). Steiermark: 2 exx., Bezirk Feldbach, Reigersberg, 320m, near pond, 14.III.1998, leg. Holzer (cAss); 1 ex., Graz (NHMW); 1 ex., Gösting near Graz (NHMW). Burgenland: 1 ex., Bruck a. d. Leitha, in rotting Salix, 1928 (SMNS); 6 exx., Neusiedler See (FIS, NHMB, SMTD); 12 exx., Neusiedlersee, leg. Bernhauer, Luze, Reitter, Scheerpeltz, 
Schuster (MNHUB, NHMW, SMTD). Locality not specified: 1 ex. (DED); 1 ex., leg. Schüppel (MNHUB).

Italy: Piemonte: 2 exx., locality not specified (FIS, SMNS). Veneto: 2 exx., Villafranca (VR), 27.IV.1982, leg. Daccordi (cWun, cZan); 1 ex., Verona, near stadium, with Lasius fuliginosus, 23.I.1975, leg. Zanetti (cZan); 3 exx., San Floriano (VR), vineyard, 8.III.\&24.IV. (UKNHM, cZan); 1 ex., same data, but 28.V. (cZan); 2 exx., same data, but 16.V. (cZan); 1 ex., Trissino (VI), meadow, nest of Talpa, 12.I.1997, leg. Tagliapietra \& Zanetti (cZan). Emilia-Romagna: 1 ex., N Modena, San Cataldo, 28.III.1897, leg. Fiori (MNHUB); 3 exx., locality illegible, 30.XI.1889 \& 11.XI.1906, leg. Fiori (MNHUB). Toscana: 10 , Torre del Lago Puccini, VII.1939, leg. Gagliardi (cAss); 1 ex., Vallombrosa, leg. Fiori (MNHUB); 1 ex., Lucca (NHMW). Lazio: 3 q q , 6 exx., Roma, leg. Luigioni (DEI, SMTD, cAss); 1 ex., Roma, 14.V.1940, leg. Frey \& Koch (NHMB); 1 ex., Roma, Aniene inundation, 2.XII.1900, leg. Luigioni (MNHUB); 1 ex., Monte Libretti, 15.IV.1975, leg. Rossi (cZan); 2 exx., locality not specified (NHMW). Sicilia: $10^{*}$, Erice (TP), mixed forest, 10.XII.1993, leg. Sabella (cAss); 10 , Segesta, V.1976 (MHNG); 1 ex., Monte Pelegrino, 1906, leg. Leonhatd (DEI); 2 exx., locality not specified (DEI, NHMW). Locality ambiguous: 1 ex., Alessio, V.1916 (NMP).

Czech Republic: Bohemia: 1 ex., České středohor̆i, v. Borač, 30.III.1994, leg. Moravec (cAss); 1 ex., České středohoři, wrch Kaná, 15.IV.1993, leg. Moravec (cAss); 6 exx., Btandýs n.L., leg. Skalitzky, etc. (NHMW); 1 ex., Praha, leg. Skalitzky (NHMW); 10 , S Praha, Modrany, leg. Klička (cAss); 1 ex., NE Praha, Celákovice, leg. Klička (NMP); 1 ex., NE Praha, Sojovice, leg. Klička (NMP); 3 exx., NE Praha, Toušen, 15.III.1906 (NMP, cAss); 1 ex., Vltava river [= Moldau], 9.III.1906 (NMP). Moravia: 1 ex., Čejč, VII.1940, leg. Hoffer (NMP); 1 ex., Dyje river, leg. Fleischer (NMP); 1 ex., Prostëjor ["Prossnitz"], leg. Zoufal (NHMW); 4 exx., locality not specified (NMP).

Slovakia: 1 ex., Kamenný Most, 3.IV.1955, leg. Bohac (cRen); 2 exx., Kamenný Most, 3.V.1955, leg. Smetana (MNHUB, cVog); 1 ex., Zliechor, 25.IV.1917 (NMP).

Hungaria: 12 exx., Szeged, 15.X.1920 (1 ex.), 21.VI.1926 (1 ex.), V.1927 (1 ex.), 8.IV.1928 (1 ex.), 17.IV.1932 (3 exx.), 8.III.1934 (1 ex.), 26.III.1934 (1 ex.), 8.III.1937 (2 exx.), leg. Stiller (HNHM, cAss); 5 exx., Kalosca (NHMW, NMP); $10^{\circ}, 3$ exx., Siófok (NMP); 2 exx., Neusiedler See (NHMW, SMTD); 1 ex., Sopron Com. (MHNG); 1 ex., Balaton (NHMW).

Croatia: 10 [with worker of Lasius fuliginosus attached to the same pin], Zagreb, leg. Weingärtner (FIS).

Bosnia-Herzegovina: 1 ex., Sarajevo, V.1907 (NMP); 10 , 1 \% , 2 exx., Sarajevo, flood (SMTD, cAss); 2 exx., Gacko, leg. Hoffmann (NHMW); 1 ex., "Pasino", Brdo, X.1931 (NHMB); 1 ex., "Bosnia, Illidze" (NMP); 3 exx., "Bosnien, Reitter.Leder" (NHMB, NHMW).

Yugoslavia: Montenegro: 1 ex. [previously identified as A. bonnairez], S Herceg-Nori, Bay of Kotor ["Bocche di Cattaro"], IV.1916, leg. Matcha (NMP); 19 [nanistic, previously identified as A. bonnairei], Kameno, leg. Paganetti (cAss). Serbia: 1 ex., Silbas ["Szilbasz"], 45²4N, 19²8E (NMP).

Albania: 1 ex., Skutari (cVog); 1 ex., Miljoti ["Miloti"], leg. Matzenauer (FIS); 1 ex., Vlorë ["Avlona"] (MNHUB).

Macedonia: 1 ex., locality not specified (NHMW).

Greece: continental: 19 , Fthiotis, Parnassos Oros, Kelatia ski resort, $38^{\circ} 33^{\prime} 02 \mathrm{~N}, 22^{\circ} 34^{\prime} 48 \mathrm{E}, 1780 \mathrm{~m}$, 15.IV.2000, leg. Assing (cAss); 1 ex., Fthiotis, Xeroruni Oros, 700-800m, sifted, 30.V.-1.VI.1933, leg. Beier (NHMW); 1 ex., same data, but 6.VL.1933 (NHMN); 1 ex., Nestos estuary, $7 \mathrm{~km}$ from mouth, 17.III.1993, leg. Sabella (cZan). Pelopónnisos: 1o, Erimanthos, Stavrodromi, 400m, 23.III.1992, leg. Kerner (cAss); 10, 1 ㅇ, Meligalas, Derreni, 600m, 26.III.1992, leg. Frisch (cWun, cAss); 1 ex., Taygetos, leg. Brenske (NHMW); 2 exx., Kiparissía, 5.-10.V.1956, leg. Schubert (NHMW); 1 ex., Yithion, 18.-29.IV.1956, leg. Schubert (cAss); 1 \% "Nordpelopones" [sic], leg. Franz (cAss). Islands: 1 ex., Lefkas, 25.-26.V.1932, leg. Beier (NHMTW); 1 ex., Lefkas, 25.V.-3.VI.1932, leg. Beier (NHMW); 7 exx., Kefallinia, leg. Paganetti (DEI, MHNG, NHMW, SMNS, SMTD, cAss); 1 \%, Kefallinia, Argostoli, 1905, leg. Leonhard (DEI); 1 ex., Kefallinia, Megalo-Viaro, forest, leg. Moczarski (NHMW); 2 exx., Kérkira [=Corfu], Val de Ropa, leg. Woetz (NHMW, cAss); 2 exx., Kérkira, leg. Breit, Reitter (NHMW). Locality not specified: 1 ex., leg. Scriba (NHMW); 1 ex. (MNHUB).

Ukraine: 1 ex., Bukowina, Tscherniwzi ["Czernowitz"] (NHMW).

Locality ambiguous, not identified, illegible, or not specified: 1 ex., "Latif Oboti" (cAss); 1 ex., "Wnhgt Dulcigno" (NHMB); 1 ex., Eyrquern (MHNG); 1 ex., "Hungaria" (FIS); 31 exx. (DEI, MNHUB, NHMN, NMP). 


\section{Redescription}

$3.0-5.0 \mathrm{~mm}$ (abdomen fully extended). Size and size-related characters subject to considerable intraspecific variation. Colour of body highly variable, too; usual coloration: head and abdominal segments III-VII, except for the hind margins, dark brown to blackish; elytra, hind margins of abdominal segments III-VII, and abdominal apex ferrugineous; pronotum ferrugineous to dark brown, in most specimens \pm castaneous; antennae (except for the lighter basal antennomeres) \pm castaneous; legs and mouthparts yellowish to yellowish brown, sometimes with the maxillary palpi partly infuscate; occasionally whole body of similar colour as in A. umbrosa, apart from the legs and mouthparts \pm completely dark brown to blackish, or of similar colour as $A$. bonnairei, with the whole body \pm uniformly dark testaceous, ferrugineous, or yellowish brown. Head, pronotum, elytra, and most of abdomen usually without appreciable microsculpture; abdominal terga VII and VIII with very shallow transverse microsculpture. Pubescence of forebody relatively long and suberect.

Head of subcircular shape, approximately as wide as or slightly wider than long; not or only indistinctly dilated behind eyes, eyes moderately large and convex, weakly projecting from lateral outline of head, their diameter approximately half the length of postgenae in dorsal view or slightly larger, the latter smoothly curved towards posterior constriction or \pm subparallel. Puncturation moderately fine and sparse, more distinct than in A. umbrosa. Genal carina distinct. Antenna long and massive, antennomere I with dorso-apical furrow of somewhat variable length and depth; antennomere III approximately as long as antennomere II (Fig. 77). Third joint of maxillary palpus very long and slender, similar to that in $A$. bonnairei (Fig. 76); labrum of similar shape of chaetotaxy as in A. bonnairei; labium as in Fig. 75 .

Pronotum approximately 1.1 times as wide as long and $1.25-1.35$ times as wide as head; maximal width in or a short distance anterior to middle; puncturation shallow, \pm similar to that of head or finer.

Elytra at suture in most specimens $0.80-0.85$ times the length of pronotum and approximately 1.20 times as wide as pronotum; posterior margin near posterior angles strongly sinuate; puncturation granulose, relatively dense and coarse, near scutellum often \pm rugose. Hind wings fully developed. Legs long and slender, first metatarsomere approximately as long as the combined length of the three following tarsomeres or only slightly shorter. Mesosternum with median carina of variable length; sometimes only a short rudiment of such a carina present near anterior margin; mesosternal process apically acute.

Abdominal terga III-V with very deep, tergum VI with moderately deep anterior impression; sterna III-VI anteriorly constricted, this constriction more pronounced on sterna III-V than on sternum VI; puncturation in anterior impressions of terga III-VI dense and coarse, on remainder of dorsal surface fine and sparse; tergum X as in Fig. 79. o: posterior margin of tergum VIII weakly convex, with a row of rather long thin marginal setae; posterior margin of sternum VIII distinctly convex, with a dense row of moderately long marginal and a sparse row of very long submarginal thin setae; aedeagus with ventral process of median lobe not distinctly separated, in lateral view weakly bent, and with distinct basal fold; crista apicalis of reduced, crista proximalis of moderate size; internal sac with relatively short tube (Figs. 71-72); apical lobe of paramere relatively slender and with external fold-like structure (Fig. 73). 

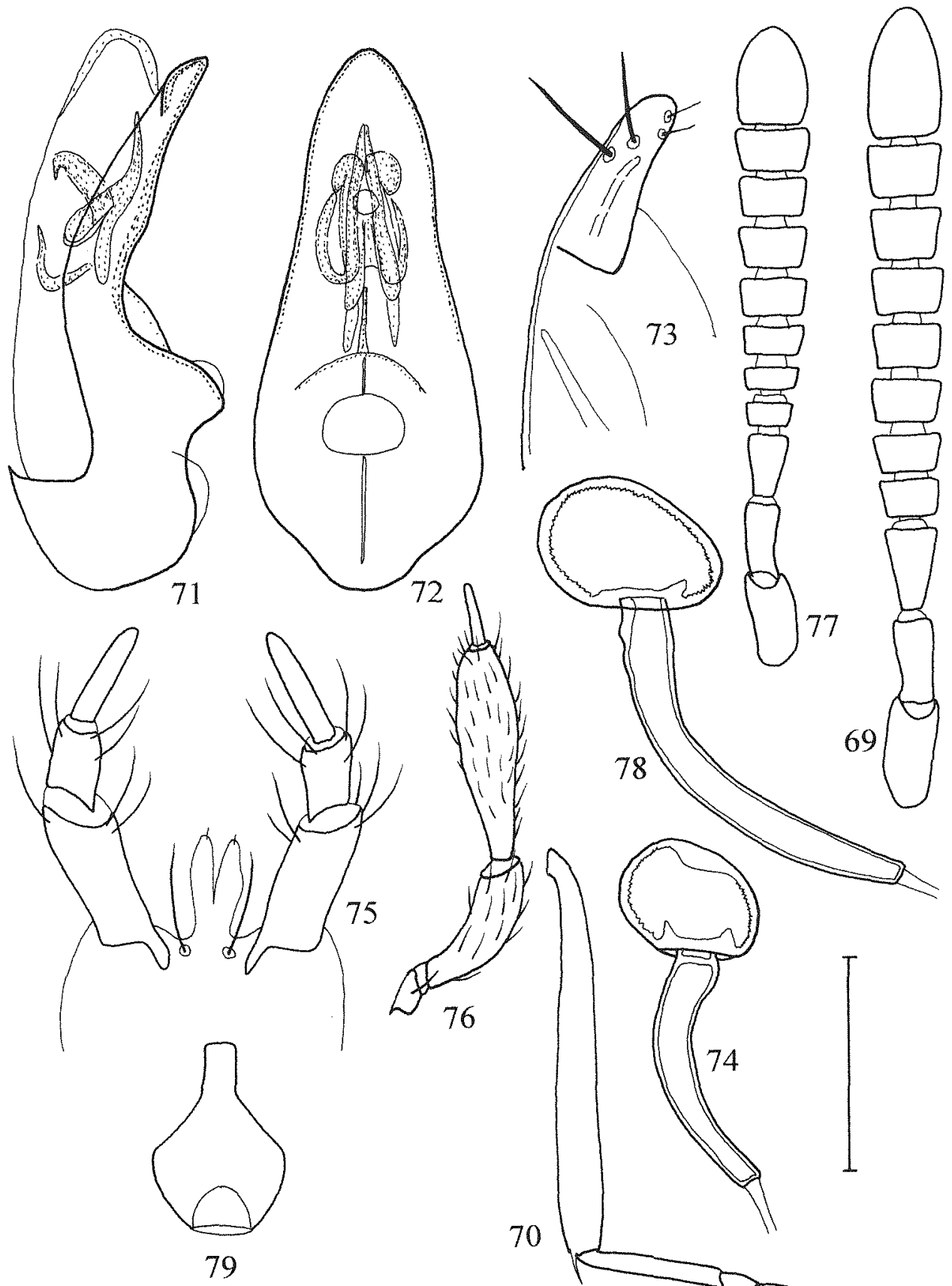

Figs. 69-79: Amarochara crassicornis (QUEDENFELDT) (69-70), A. forticomis (LACORDAIRE) (71-77), and $A$. carinata sp. n. (78-79). Antenna, pubescence omitted $(69,77)$; metatibia and metatarsus, pubescence omitted (70); median lobe of aedeagus in lateral and in ventral view $(71,72)$; apical lobe of paramere (73); spermatheca $(74,78)$; labium (75); maxillary palpus (76); tergum X, pubescence omitted (79). Scale: 73,75 : $0.1 \mathrm{~mm} ; 71,72,74,76,78: 0.2 \mathrm{~mm} ; 69,70,77,79: 0.4 \mathrm{~mm}$. 
OP: tergum VIII of similar shape and chaetotaxy as in $0^{*}$; posterior margin of sternum VIII weakly convex, row of marginal setae very weakly modified, relatively thin, and very short; spermatheca as in Fig. 74 .

\section{Intraspecific variation}

Coloration and all size-related parameters may vary considerably. Nanistic specimens were seen from various regions, especially from the Balkans. The shape of the posterior margin of the elytra and the depth of the anterior impression of the abdominal tergum VI, too, are subject to some intraspecific variation.

\section{Comments}

The original description is based on an unspecified number of syntypes ("Rare. Fontainebleau"). Repeated requests for a loan of the types, which are deposited in the Muséum National d'Histoire Naturelle Paris, have remained unanswered.

\section{Comparative notes}

A. forticornis is distinguished from all congeners occurring in the Holarctic region especially by the presence of a distinct anterior impression on the abdominal tergum VI, by the strongly sinuate posterior margin of the elytra, and by the male primary sexual characters (morphology of the median lobe of the aedeagus and of the apical lobe of the paramere. From all species, except $A$. carinata, it is additionally separated by the presence of a median carina on the mesosternum.

\section{Distribution and bionomics}

Like A. bonnairei, A. forticornis is apparently a Ponto-Mediterranean element (see LATTIN, 1967), its distribution ranging from (northern?) Spain, France, southern England, southern Scandinavia, and Estonia in the west, northwest, and north, to southern Italy, southern Greece, and Ukraine in the south, southeast, and east (Map 6) (material examined and FOXXER, 1888, HABERMAN, 1983, HORION, 1938, JOY, 1932, LuIgIONI, 1929, LUNDBERG, 1995, SILFVERBERG, 1992, ZANETTI, 1995). The species is also indicated from the Caucasus region and from Turkey (BerNHAUER, 1902, Horion, 1967), but these records require confirmation.

Only two specimens were seen from Spain; the precise locality, however, is not specified on the labels. OUTERELO (1980) reports the species from southern Spain (Sierra de Cazorla, under pine bark), but this record is based on a misidentification and in fact refers to a species of Pbloeopora ERICHSON (specimen examined). In France, the species is apparently widespread, but rare (material examined; Horion, 1967); it has also been reported from Corsica (Luigioni, 1929, SAINTE-Claire Deville, 1914). In England and Scandinavia, its known distribution is confined to the south (FOWLER, 1888, HORION, 1967, JoY, 1932, LundBerG, 1995, PALM, 1972). It has become known from various regions in Denmark (HANSEN, 1964, HANSEN, 1996) and was rather recently discovered also in Estonia (HABERMLAN, 1983).

In Germany, it is present in almost all regions (see material examined) and here also recorded from Mecklenburg-Vorpommern, from where it was previously unknown (KÖH- 
LER \& KLAUSNITZER, 1998); for additional records see KOCH (1968), KÖHLER (1997), LINKE (1907, 1927, 1962), LOHSE (1967), SCHOLZE \& JUNG (1994), and STRZELCZYK (1988).

A. forticomis is here - apparently for the first time - recorded from Switzerland; neither HORION (1967) nor LUCHT (1987) indicate any Swiss records. Material from eastern and southeastern Austria (Niederösterreich, Steiermark, Burgenland) was examined; HORION (1967) and SCHEERPELTZ (1968) also report the species from Vorarlberg, Oberösterteich, and Kärnten, but the corresponding specimens were not found in the Scheerpeltz collection, where they are supposedly kept (HORION, 1967). In Italy, it is distributed from the north to the extreme south, including Sardinia and Sicily (material examined; LuIgIONI, 1929, PORTA, 1926, ZANETTI, 1995).

A. forticornis has repeatedly been recorded from the Czech Republic, Slovakia, Hungary, and Romania; for additional records see ÁDÁM (1987), BOHÁČ et al. (1993), HORION (1967), KOCIAN (1993), VÁSÁRHELYI (1985). Records from the Balkans are relatively scarce (see material examined and COIFFAIT (1976), NOVAK (1952), and SCHEERPELTZ (1959, 1967)).

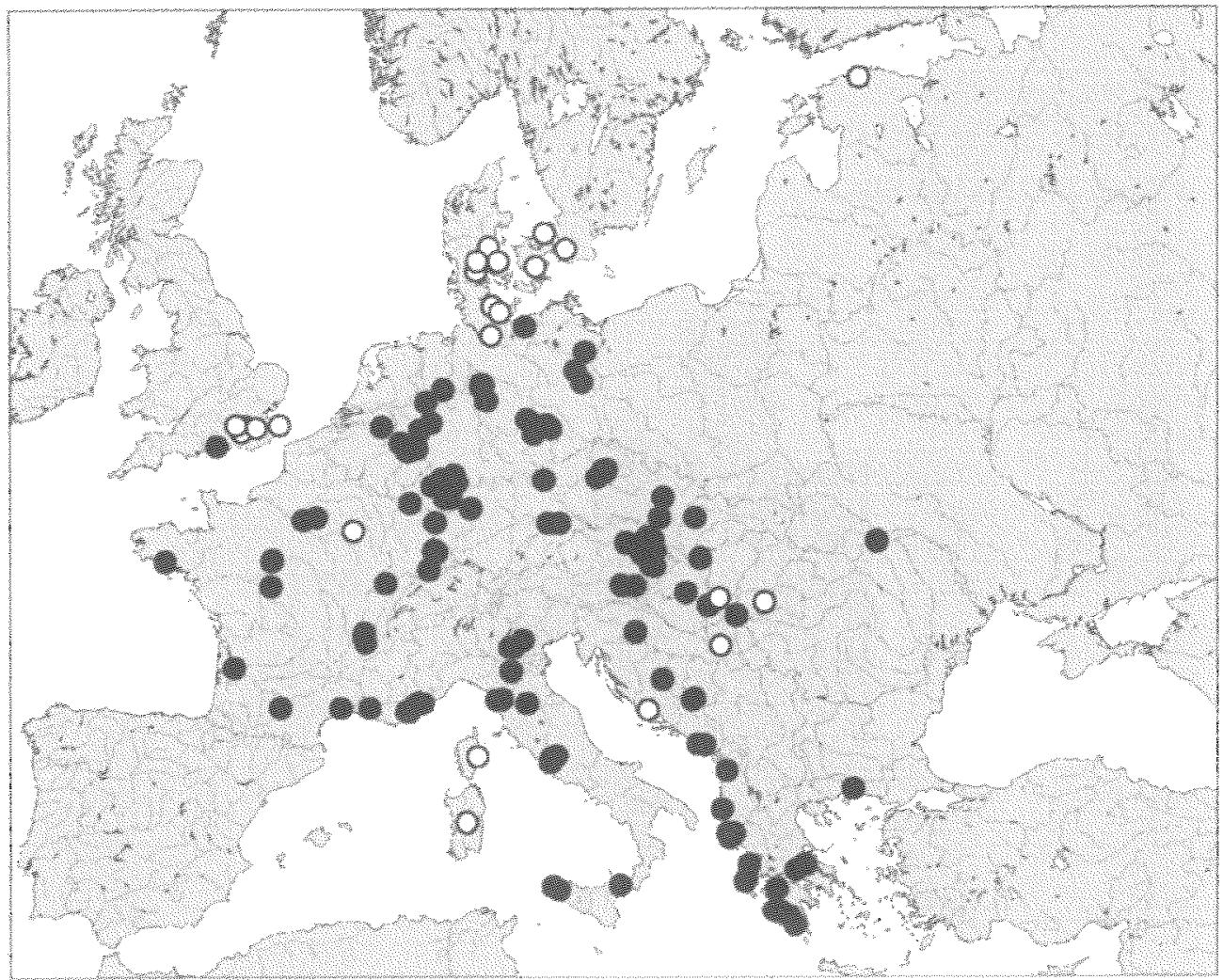

Map 6: Distribution of Amarochara forticornis (LACORDAIRE) based on revised records (filled circles) and selected literature records (open circles). 


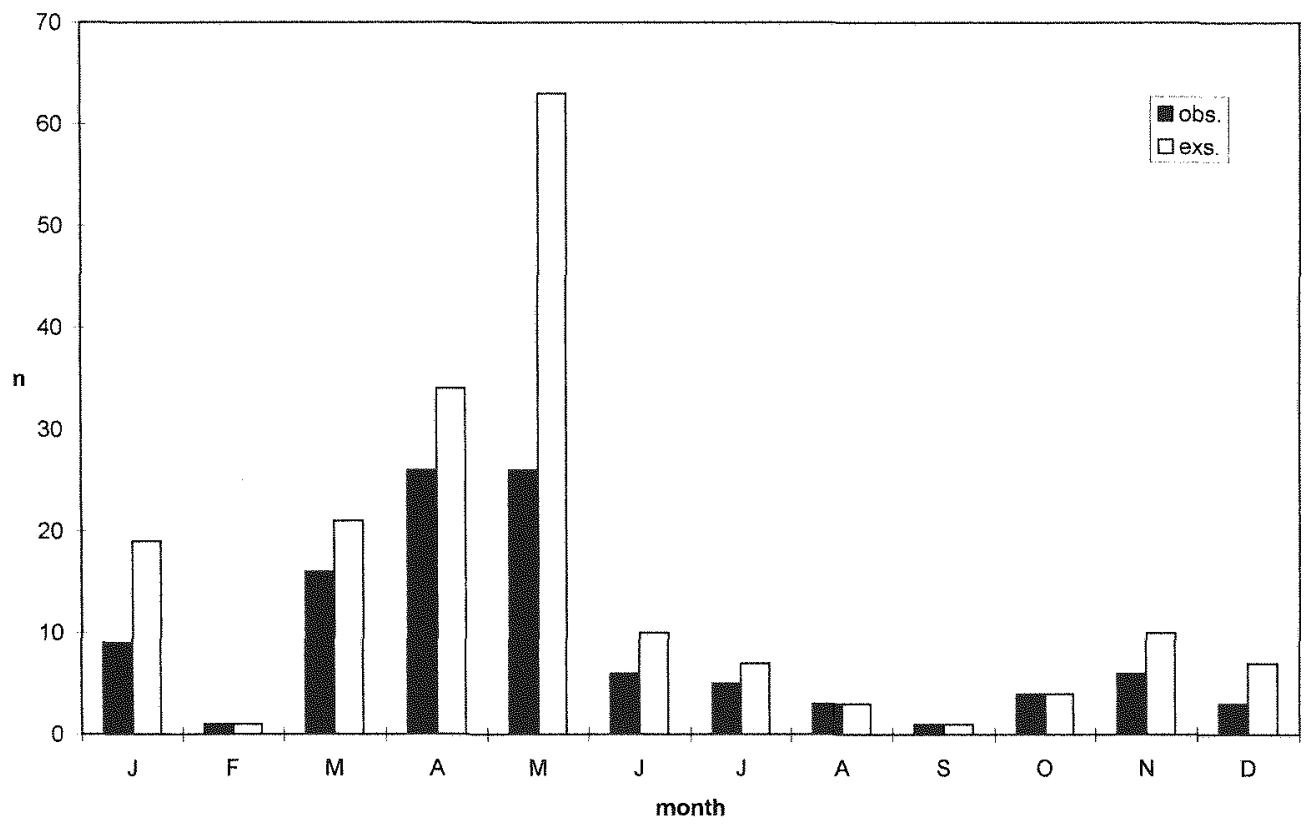

Fig. 80: Amarochara forticornis (LACORDAIRE). Seasonal phenology based on material examined. Abbreviations: obs. $=$ number of observations or samples; exs. = number of specimens.

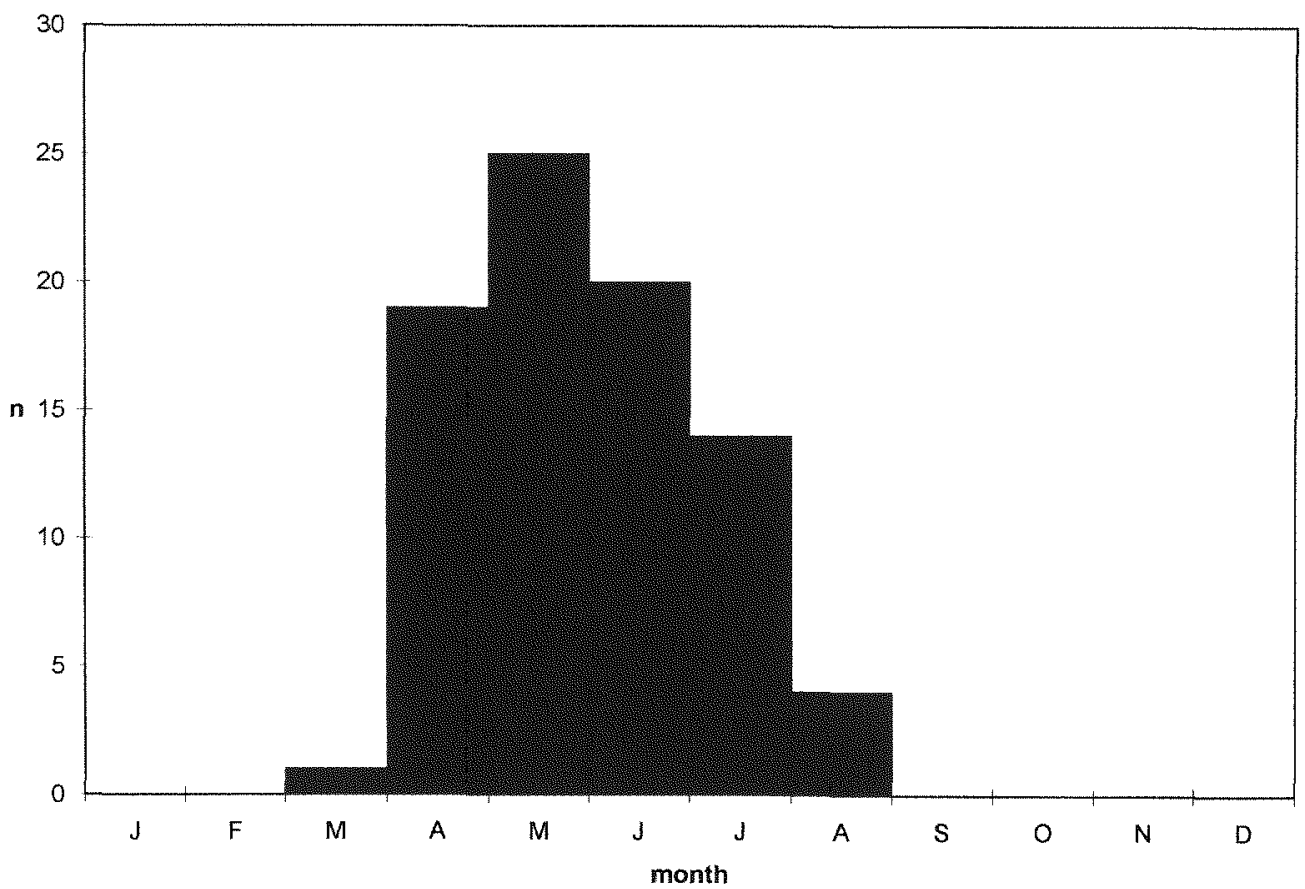

Fig. 81: Amarochara forticornis (LACORDAIRE). Seasonal epigeic activity based on pitfall trap studies in the surroundings of Hannover, Germany (55 pitfall traps). 
As in the case of $A$. umbrosa and $A$. bonnairei, the species was observed in a wide range of biotopes, but reproduction and hibernation take place in an unknown subterranean habitat. Based on the material examined (additional literature data given in brackets), the species was found in various forest types (flood-plain, mixed, and deciduous woodland), on or near banks of ponds, lakes, and rivers (HORION, 1967), in ditches, in xerothermous grassland (ASSING 1994a, HORION, 1967), on one occasion in the alpine region near a snowfield, several times in arable land (HORION, 1967), vineyards, in sand and gravel pits (FOVRLER, 1888, KÖHLER, 1997), and often also in urban habitats (fallows, gardens, etc.) (AsSING, 1988, KACHE, 1998, STRZELCZYK, 1988). The vast majority of records are from lower elevations, but one Greek specimen was taken at an altitude of almost $1800 \mathrm{~m}$.

A. forticornis has been observed throughout the year, with a maximum in spring (Fig. 80). In spring and summer, the species is usually collected on the ground surface by pitfall trapping or by manual sampling methods, whereas nearly all the late autumn and winter records (November through March) are from flood debris, suggesting that the beetles were washed out of their cryptic hibernation habitat. Some winter records are also from ant nests (Lasius platytborax, L. brunneus, L. fuliginosus) and from mole nests; for additional data see HORION (1967) and OSELLA \& ZANETTI (1975). Remarkably, one specimen was found in December using a floating technique (soil-washing) used for collecting endogean beetle species (see material examined). Pitfall trap studies showed that epigeic activity begins in March and ends in summer, with a maximum from April through June (Fig. 81). From the observations that the species may be caught in greater numbers by pitfall trapping in spring, but is rarely found by sifting or other manual samp-ling techniques or during other seasons of the year, it can be inferred that the beetles are epigeically active only to search for suitable (subterranean) reproduction habitats and/or for mates. Dispersal activity by flight was recorded with malaise traps and car-nets in May and August (material examined; KÖHLER, pers. comm.). However, no flight muscles were found in four dissected beetles collected by pitfall trapping, suggesting that $A$. forticornis may be dimorphic regarding its flight-muscle development. One female caught in April had a mature egg in the ovaries; teneral adults were observed in August.

\subsubsection{Amarochara carinata sp. $\mathrm{n}$.}

Figs. 78-79

\section{Type}

Holotype \%: E - LEON 1900m, Pena Trevinca- Pena Surbia, 28.5.1996, Starke leg. / Holotypus + Amarochara carinata sp. n. det. V. Assing 2000 (cAss).

\section{Description}

$4.2 \mathrm{~mm}$ (abdomen fully extended). Pronotum, elytra, legs, mouthparts, clypeus, anterior part of frons, antennomeres I and II, margins ot abdominal terga, and abdominal apex yellowish brown to rufous; apical part of antenna brown; posterior part of head dark brown; abdominal terga III-VII, except for the lighter margins, blackish. Head, pronotum, elytra, and most of abdomen without appreciable microsculpture; traces of shallow microsculpture present only on the preapical abdominal terga. Pubescence of forebody shorter, less erect, and less conspicuous than in $A$. forticornis. 
Head of subcircular shape, approximately as wide as long; weakly dilated behind eyes; eyes slightly smaller than in A. forticornis, weakly projecting from lateral outline of head, their diameter approximately half the length of postgenae in dorsal view, the latter more distinctly convex than in A. forticornis, smoothly curved towards posterior constriction. Puncturation finer, shallower, and less distinct than in A. forticornis. Genal carina present. Antenna long and massive, of similar morphology as in A. forticornis, but antennomere III wider and more strongly dilated apically, antennomere IV slightly wider and more transverse, $\mathrm{V}$ wider, more transverse, and only slightly longer than IV; antennomeres VI - X more transverse and wider than in A. forticornis, X approximately 1.5 times as wide as long, and XI longer than the combined length of IX and X. Mouthparts similar to those in A. forticornis.

Pronotum 1.13 times as wide as long and 1.3 times as wide as head; maximal width a short distance anterior to middle; puncturation fine, more distinct than that of head, and more clearly defined than in average $A$. forticornis.

Elytra shorter and narrower in relation to pronotum than in $A$. forticornis; at suture 0.74 times the length of pronotum and approximately 1.1 times as wide as pronotum; posterior margin near posterior angles weakly sinuate; puncturation slightly granulose, somewhat denser than in A. forticornis, near scutellum denser, but not rugose. Legs long and slender; metatarsi longer and more slender than in A. forticornis, first metatarsomere distinctly longer than the combined length of the three following tarsomeres. Mesosternum with median carina; mesosternal process apically acute.

Abdominal terga IIII-V with anterior impressions of similar depth as in $A$. bonnairei, much less deep than in A. forticornis; tergum VI with shallow anterior impression; sterna III-VI anteriorly constricted, this constriction more pronounced on sterna III-V than on sternum VI; punctures in anterior impressions of terga III-VT distinct and larger than in $A$. bonnairei, but much less coarse than in $A$. forticomis; puncturation on posterior parts of terga III-VII, however, distinctly denser and coarser than in A. forticornis; tergum $\mathrm{X}$ as in Fig. 79.

ot: unknown.

O: posterior margin of tergum VIII moderately convex and with row of relatively long setae; posterior margin of sternum VIII, with row of short and moderately modified marginal setae; spermatheca as in Fig. 78.

Derivatio nominis: The name (Lat., adj.) refers to the presence of a median carina on the mesosternum, which distinguishes the species from all Palaearctic and Nearctic congeners, except for $A$. forticornis.

\section{Comparative notes}

From $A$. forticornis, the species is readily distinguished especially by the lighter coloration of the forebody, the wider and more transverse antennomeres, the relatively shorter and narrower elytra, the weakly sinuate elytral hind margin, the longer metatarsomere I, the distinctly shallower anterior impressions of the abdominal terga III-VI, and by the completely different puncturation of the abdomen (see description above). From the similarly coloured $A$. bonnairei, it differs in the larger size, the much more massive antennae, the presence of a mesosternal carina, the relatively larger and flatter pronotum, the much longer metatarsomere I, and by the much coarser abdominal puncturation. 


\section{Distribution and bionomics}

The species is known only from one locality in northern Spain, where it was collected at an altitude of $1900 \mathrm{~m}$.

\subsection{The loebli group}

Species of the loebli group occur in the Eastern Palaearctic region and have been recorded from the Himalayas, China, and Taiwan. The monophyly of this group is secured by several significant synapomorphies, i. e. a posteriorly constricted head with a distinct neck, broad connecting joints between the antennomeres IV-XI, a short ventral process of the median lobe of the aedeagus, and by the derived morphology of the apical lobe of the paramere. In addition, they share the following characters, some of them synapomorphic, but shared also with other species groups, and some plesiomorphic: genal carinae absent, eyes relatively large, antennomere III shorter than II, third joint of maxillary palpus long and slender, ligula only apically bifid, long and slender mandibles, pronotum slender and with relatively sparse puncturation, mesosternum without carina, mesosternal process apically broadly truncate, tarsi long and slender, anterior impressions of abdominal terga III-V deep and coarsely punctate, posterior parts of abdominal terga III-VI sparsely punctate, abdominal tergum $\mathrm{X}$ with numerous stout setae also in the median area and with relatively short anterior extension, internal sac of aedeagus with distinctly sclerotized internal structures (see also section 3.3).

\subsubsection{Amarochara loebli PACE, 1992}

Figs. 82-91, Map 7

Amarochara (s. str.) loebli PACE, 1992: $282 \mathrm{f}$.

\section{Type examined}

Holotype $\sigma^{*}$ [elytra and one antenna missing]: NEPAL (Prov. Bagmati), Tarke Ghyang, 2650m, 19.IV.81, Löbl \& Smetana / HOLOTYPUS Amarochara loebli m. det. R. Pace 1988 / Amarochara loebli sp. n. det. R. Pace 1988 (MHNG).

\section{Additional material examined}

1 i , Nepal, Kathmandu, Dis. Siwapuri Dara, 2300m, 3.V.1985, leg. Smetana (MHNG); 1 \%, same data, but 2400m, 30.TV.1985 (MHNG); 10 , Khandbari District, forest above Ahale, 2200m, 4.IV.1984, leg. Smetana \& Löbl (cAss); 1 q [with mature egg in ovaries], Khandbari District, Induwa Khola Valley, 2850m, 15.IV.1984, leg. Smetana \& Löbl (MHNG); 4 \& [1 9 teneral, $2 \%$ with mature egg in ovaries], Nepal, Sankhua Sabha District, above Pahakhola, 2600-2800m, 31.V.-3.VI.1988, leg. Schawaller (SMNS).

\section{Redescription}

2.9 - $3.3 \mathrm{~mm}$. Head dark brown; pronotum brown, elytra slightly lighter, yellowish brown to light brown; abdomen brown, with the tergal margins and the abdominal apex lighter; antenna dark brown, with antennomeres I-II or I-III light brown to brown; legs yellowish 
brown to brown. Head with extremely shallow, barely noticeable microsculpture, apical abdominal terga with very fine and shallow transverse microstriae; remainder of dorsal surface without microsculpture and shining. Pubescence of forebody moderately long and \pm suberect.

Head of subcircular shape, slightly wider than long, not dilated behind eyes; posteriorly with distinct and slender neck of approximately half the width of head; eyes large and convex (but less so than in A. umbrosa), weakly projecting from lateral outline of head, their diameter slightly shorter than postgenae in dorsal view, the latter smoothly curved towards posterior constriction, posterior angles \pm obsolete. Puncturation extremely fine and sparse, barely noticeable. Genal carina absent. Antenna massive, antennomere I with dorso-apical furrow of somewhat variable length and depth, similar to that in $A$. umbrosa; antennomere III slightly shorter than II; connecting joints between antennomeres broad, more so than in W-Palaearctic congeners (Fig. 90). Maxillary palpi of similar morphology as in A. umbrosa, but fourth joint slightly longer (Fig. 89); ligula apically rounded, not distinctly bifid; apical joint of labial palpi very long and slender (Fig. 88); mandibles very long and slender, more so than in $A$. umbrosa.

Pronotum approximately $1.10-1.15$ times as wide as long and $1.24-1.31$ times as wide as head; maximal width in or a short distance anterior to middle; puncturation shallow, fine, and ill-defined.

Elytra at suture $0.85-0.90$ times the length of pronotum and approximately $1.25-1.30$ times as wide as pronotum; posterior margin near posterior angles not distinctly sinuate; puncturation fine (but more distinct than that of head and pronotum), indistinctly granulose, somewhat sparser than in A. umbrosa. Legs relatively longer than in A. umbrosa; first metatarsomere at least slightly longer than the combined length of the two following tarsomeres, sometimes as long as the combined length of the three following tarsomeres. Mesosternum without median carina; mesosternal process apically broadly truncate (much broader than in $A$. umbrosa).

Abdominal terga III-V with deep (slightly deeper and more well-defined than in $A$. umbrosa), tergum VI without anterior impression; sterna III-V anteriorly constricted; puncturation in anterior impressions of terga III-V and near anterior margin of tergum VI very dense and coarse, on remainder of tergal surface very fine and sparse. Tergum $\mathrm{X}$ anteriorly not distinctly elongated, narrowly truncate (Fig. 91); with very dense stout setae.

$o^{\lambda}$ : posterior margin of tergum VIII truncate, with a row of moderately long thin marginal setae; posterior margin of sternum VIII convex, with a dense row of moderately long and thin marginal setae and with interspersed long thin submarginal setae; aedeagus with short, slender, and, in lateral view, weakly bent ventral process; at base of ventral process with distinct median process; crista apicalis reduced, crista proximalis long, but not very prominent; internal sac with short tube and with additional pairs of long, moderately sclerotized structures (Figs. 82-83, 86); paramere strongly modified, apical lobe of paramere separated from basal part of paramerite by membrane, strongly bent, flattened, with torsion, and apically broadly rounded (Figs. 84-85).

q: tergum VIII of similar shape and chaetotaxy as in $\delta^{*}$; posterior margin of sternum VIII weakly convex, row of marginal setae unmodified, thin, and short; spermatheca as in Fig. 87. 


\section{Comparative and systematic notes}

From all Western Palaearctic congeners, $A$. loebli is readily distinguished by the presence of a distinct slender neck. For distinction from other species with a distinct neck see the species sections below. As can be inferred from the morphology of the aedeagus, $A$. loebli is the adelphotaxon of A. armata (see section 4.4.3).

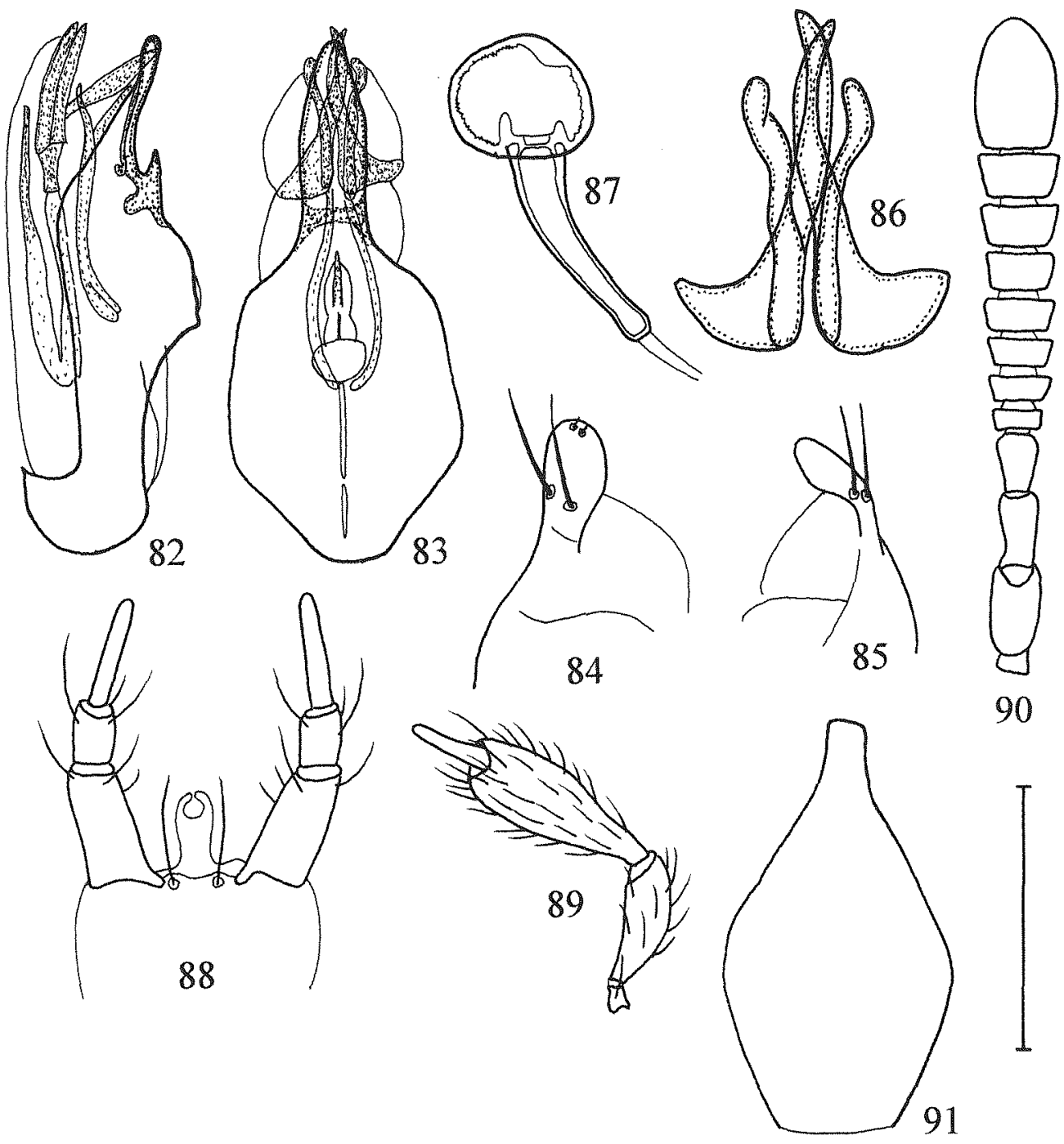

Figs. 82-91: Amarochara loebli PACE. Median lobe of aedeagus in lateral and in ventral view (82, 83); apical lobe of paramere in two different aspects $(84,85)$; apical internal structures of aedeagus in ventral view (86); spermatheca (87); labium (88); maxillary palpus (89); antenna, pubescence omitted (90); tergum X, pubescence omitted (91). Scale: $84-86,88: 0.1 \mathrm{~mm} ; 82,83,87,89,91: 0.2 \mathrm{~mm} ; 90: 0.4 \mathrm{~mm}$. 


\section{Distribution and bionomics}

This species, which was previously known only from the type locality in the Bagmati province, is here recorded also from Kathmandu, from the Sankhua Saba District and from the Khandbari District in Nepal (Map 7). The holotype and the additional specimens examined were collected in April and May at altitudes of $2200-2850 \mathrm{~m}$. The ovaries of three females taken in April and in the beginning of June contained mature eggs.

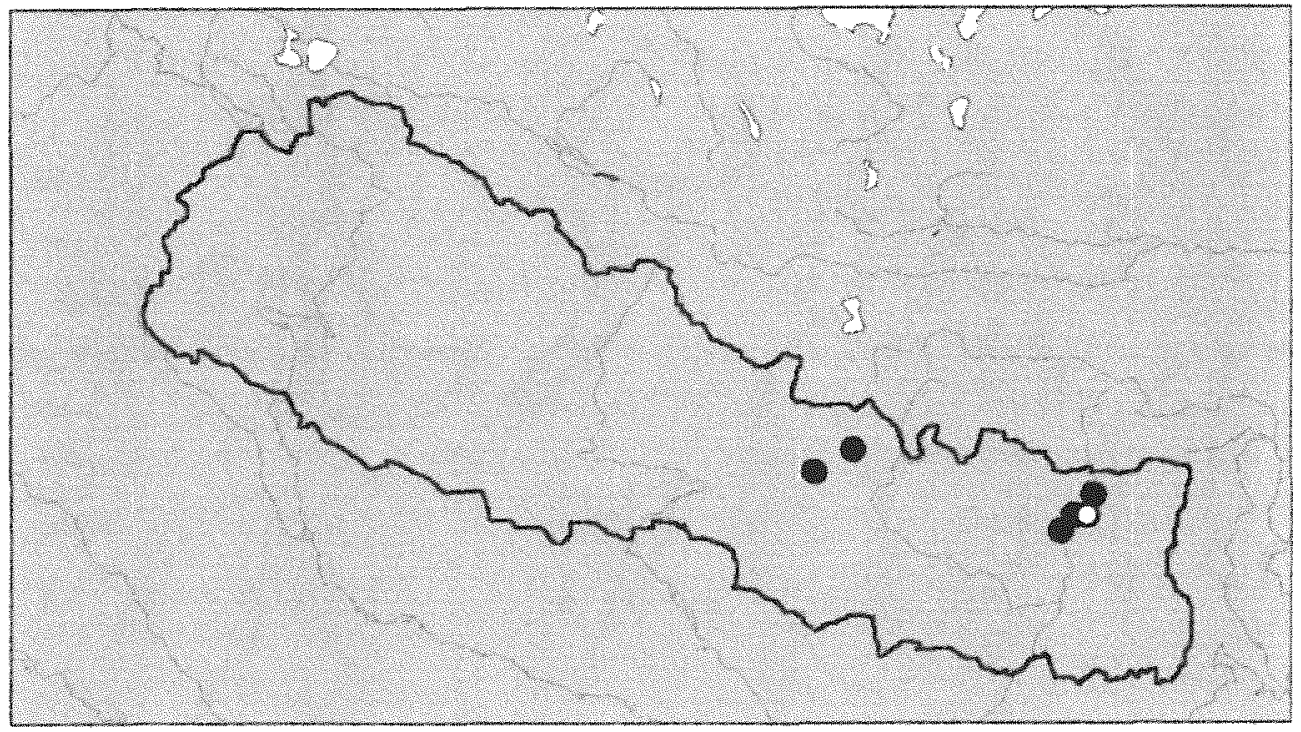

Map 7: Distribution of Amarocbara loebli PACE (filled circles) and A. seriepunctata sp. n. (open circle) in Nepal.

\subsubsection{Amarocbara seriepunctata sp. $\mathrm{n}$. Map 7}

\section{Type}

Holotype + [slightly damaged]: E. NEPAL: KOSI, Val. Arun ss/Num, 1100m, 21.IV.84, Löbl - Smetana / Holotypus o Amarochara seriepunctata sp. n. det. V. Assing 2000 (MHNG).

\section{Description}

Highly similar to $A$. Loebli, distinguished only by the following characters:

Head slightly more transverse, approximately 1.1 times as wide as long; pronotum distinctly transverse, approximately 1.15 times as wide as long; legs distinctly shorter, length of metatibia $0.39 \mathrm{~mm}$ (in $A$. loebli $0.48-0.52 \mathrm{~mm}$ ).

Abdominal terga III and IV with one, tergum $V$ with two conspicuous transverse series of dense and coarse punctures in posterior half. 
§: unknown.

\%: posterior margin of tergum VIII distinctly convex and with longer marginal setae; posterior margin of sternum VIII more strongly convex; a spermatheca was not found in the apparently teneral holotype.

\section{Derivatio nominis}

The name (Lat., adj.) refers to the distinctive transverse series of dense and coarse punctures on the abdominal terga III-V.

\section{Comparative notes}

A. seriepunctata is separated from all other species of the Holarctic region by the characteristic puncturation of terga III - V. For additional characters distinguishing it from $A$. loebli and other Eastern Palaearctic congeners see description above and the comparative notes in the following species sections, respectively. The phylogenetic affiliations to the other species of the loebli group are uncertain, particularly because the male sexual characters are unknown.

\section{Distribution and bionomics}

The species is known only from the type locality in eastern Nepal, where it was collected in April at an altitude of only $1100 \mathrm{~m}$.

\subsubsection{Amarochara armata sp. n.}

Figs. 92-95, Map 8

\section{Types}

Holotype $0^{*}$ : CHINA W.Sichuan (Ganzi Tibet. Aut. Pref, Luding Co.) W Erlang Shan Pass, 2600 m, $7 \mathrm{~km}$ SSE Luding, 2951N/102¹5E, 20.-29.VI.1999, D.W. Wrase / Holotypus o Amarochara armata sp. n. det. V. Assing 2000 (cAss). Paratype ơ: CHINA: Shaanxi Prov., Taibai Shan above Houshenzi, $1300-1700 \mathrm{~m}$, 9.VI.-3.VII.1998, leg. P. Jäger \& J. Martens (SMNS).

\section{Description}

3.2-3.9 $\mathrm{mm}$. In external appearance highly similar to A. loebli, but distinguished as follows:

Coloration as in dark $A$. Loebli. Antenna equally massive, but somewhat longer; antennomere I with dorso-apical furrow of variable length. Meso- and metasternal process broadly truncate (holotype) or very narrowly truncate, almost acute (paratype). First metatarsomere shorter than the combined length of the three following tarsomeres.

Anterior impressions of abdominal terga III-V in the holotype slightly more pronounced, wider, and with more and slightly coarser punctures. Tergum $\mathrm{X}$ with less dense and less stout setae. 
$0^{\pi}$ : posterior margin of tergum VIII almost truncate and with row of rather long marginal setae; sternum VIII posteriorly strongly convex, almost pointed, and with dense row of relatively long thin setae; aedeagus of similar general morphology as in $A$. Loebli; ventral process of median lobe short in relation to median lobe and in ventral view of triangular shape; at base of ventral process with weak and obtuse median process; crista apicalis reduced; crista proximalis very long and prominent; internal sac with several pairs of large and distinctly sclerotized structures; internal tube relatively short (Figs. 9293); paramere as in $A$. loebli strongly modified (Fig. 94); apical lobe of paramere flattened, transverse, and apically truncate (Fig. 95).

q: unknown.

\section{Derivatio nominis}

The name (Lat., adj.) refers to the distinctive set of sclerotized structures in the internal sac.

\section{Intraspecific variation, comparative notes, and phylogenetics}

The holotype is much larger, somewhat darker, has longer first metatarsomeres, and, above all, has an apically broadly truncate meso- and metasternal process, whereas in the paratype, the meso- and metasternal process is almost acute. In both specimens, however, the - highly derived and very distinctive - morphology of the aedeagus is identical, so that they are here attributed to the same species. The paratype is apparently a nanistic specimen, the reduction in size presumably also involving changes in other external characters.

Based on external characters alone, this species is difficult to distinguish from $A$. Loebli (see description above). The morphology of the aedeagus, however, is highly distinctive and separates this species from all known congeners. The similarly highly derived morphology of the median lobe of the aedeagus and of the paramera suggest that $A$. armata and $A$. loebli are adelphotaxa.

\section{Distribution and bionomics}

The species is known from Shaanxi and Sichuan (China) (Map 8), where it was collected at altitudes between 1300 and $2600 \mathrm{~m}$. The holotype was sifted in a damp degraded deciduous forest near a stream (SCHÜLKE, pers. comm.).

\subsubsection{Amarochara wrasei sp. n.}

Figs. 96-99, Map 8

\section{Types}

Holotype o: CHINA S.Sichuan (Ya'an Pref., Shimian Co.) Xiaoxiang Ling, side-valley above Nanya Cun nr. Caluo, $11 \mathrm{~km}$ S Shimian, ca. 1250m, 7.VII.1999, D.W. Wrase / Holotypus o Amarochara wrasei sp. n. det. V. Assing 2000 (cAss). Paratypes 1 q : same data as holotype (cSch); 19 : same date and locality, but leg. Pütz (cAss); 1 q : CHINA: 
S-Shaanxi (Qinling Shan), pass on rd. Zhouzhi - Foping, $105 \mathrm{~km} \mathrm{SW} \mathrm{Xi'an,} \mathrm{N-slope,}$ $1700 \mathrm{~m}, 33^{\circ} 46 \mathrm{~N}, 107^{\circ} 58 \mathrm{E}$, leg. M. Schülke [C01-02] / 3.VII.2001, small creek valley, mixed deciduous forest, moss [sifted] [C01-02] (cSch).

\section{Description}

$3.1-3.3 \mathrm{~mm}$. In external appearance highly similar to $A$. loebli, but distinguished as follows:

Coloration as in dark A. loebli. Head distinctly transverse, $1.13-1.17$ times as wide as long. Antenna conspicuously massive, more so than in $A$. loebli, antennomeres IV-X wider and more transverse, IV and V almost disc-like and approximately 4 times as wide as long; antennomere I with distinct dorso-apical furrow. First metatarsomere approximately as long as or slightly shorter than the combined length of the three following tarsomeres.

Abdomen as in A. loebli.

$0^{*}$ : posterior margin of tergum VIII weakly convex and with row of rather long marginal setae; sternum VIII posteriorly convex and with moderately dense row of relatively long thin setae; aedeagus with ventral process longer in relation to median lobe than in A. loebli, at base of ventral process without distinct median process; crista apicalis reduced; crista proximalis long, but not very prominent; internal sac with distinctive apical pair of sclerotized structures; internal tube of similar length as in $A$. Loebli (Figs. 96-97); paramere of similar general morphology, but less strongly modified than in $A$. loebli; apical lobe of paramere flattened, oblong, slightly curred, and apically rounded (Fig. 98).

o : tergum VIII posteriorly almost truncate and with row of sparse and rather long thin marginal setae; posterior margin of sternum VIII weakly convex, with row of dense and short, distinctly modified marginal setae and with interspersed long thin setae; spermatheca with longer duct than in $A$. loebli (Fig. 99).

\section{Derivatio nominis}

The species is dedicated to David W. Wrase (Berlin), specialist of Carabidae, who collected two of the types.

\section{Comparative notes}

From the similar $A$. loebli and $A$. armata, $A$. wrasei is distinguished by the more transverse head, the more massive antennae (more strongly transverse antennomeres IV-X), and especially by the morphology of the aedeagus.

\section{Distribution and bionomics}

The types were sifted in southern Sichuan (Map 8) and in southern Shaanxi at an altitude of 1250 and $1700 \mathrm{~m}$. 

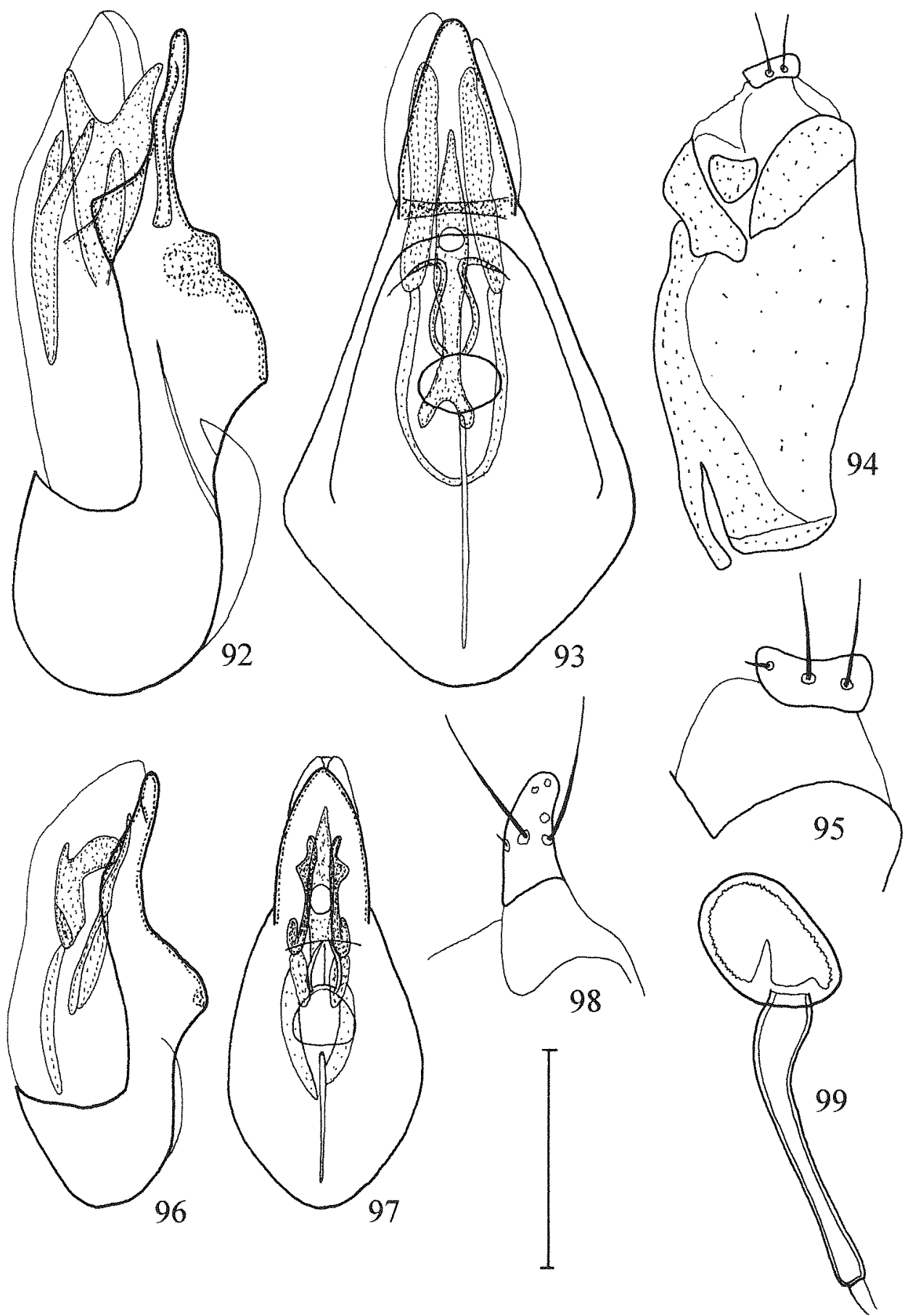

Figs. 92-99: Amarochara armata sp. n. (92-95) and A. wrasei sp. n. (96-99). Median lobe of aedeagus in lateral and in ventral view $(92,93,96,97)$; paramere (94); apical lobe of paramere $(95,98)$; spermatheca (99). Scale: $95,98: 0.1 \mathrm{~mm} ; 92-94,96,97,99: 0.2 \mathrm{~mm}$. 


\subsubsection{Amarochara megalops sp. n.}

Figs. 100-103, Map 8

\section{Types}

Holotype o*: TAIWAN Nantou Hsien, Shanlinchi, 1650m, 16.V.1990 A. Smetana [T60] / Holotypus o Amarochara megalops sp. n. det. V. Assing 2000 (MHNG). Paratypes: 1 \%: same data as holotype (MHNG); $10^{\star}$ [right antenna and antennomere XI of left antenna missing]: NEPAL Khandbari Distr. Induwa Khola Valley, 2050m, 16.IV.84, Smetana \& Löbl (cAss).

\section{Description}

3.5 - $3.6 \mathrm{~mm}$. In external appearance similar to $A$. loebli, but distinguished as follows:

Coloration as in dark $A$. loebli; base of antenna not lighter than apical antennomeres. Head transverse, nearly 1.10 times as wide as long; neck relatively narrower. Eyes very large, distinctly larger than in $A$. loebli, their diameter approximately as long as postgenae in dorsal view or nearly so; maximal width of head actoss eyes; outline of postgenae in dorsal view very weakly convex, almost straight, hind angles completely obsolete. Antenna longer than in $A$. loebli, antennomere III more slender and only slightly shorter than II, antennomeres V-X less transverse and longer; dorso-apical furrow of antennomere I longer and more pronounced than in $A$. loebli.

Pronotum with maximal width clearly in anterior half; posterior angles obtuse, but welldefined; dorsal surface with or without very shallow traces of microsculpture; pubescence somewhat longer and more erect than in $A$. loebli. Pubescence of elytra denser and more distinct. Legs longer and stouter than in $A$. loebli. First metatarsomere as long as the combined length of the three following tarsomeres or nearly so.

Anterior impressions of terga III-V deeper and broader than in $A$. loebli and with more and coarser punctures (similar to A. armata); punctures in posterior areas of terga III-V coarse and very distinct, much coarser than in $A$. loebli, sometimes forming irregular transverse rows.

$0^{*}$ : posterior margin of tergum VIII weakly convex, almost truncate, and with row of rather long and dense marginal setae; sternum VIII posteriorly moderately convex and with dense row of relatively long thin setae; aedeagus with ventral process of median lobe longer than in $A$. loebli, but less slender, and at base without median process; crista apicalis and crista proximalis small; internal tube relatively shorter and other internal structures of different shape (Figs. 100-101); paramere less modified than in A. loebli, apical lobe of paramere less flattened, not distinctly bent, and more slender (Fig. 102). q: tergum VIII posteriorly weakly convex, almost truncate, and with tow of dense and rather long thin marginal setae; posterior margin of sternum VIII moderately convex, with row of dense and short, weakly modified marginal setae and with interspersed long thin setae; spermatheca larger and with longer duct than in A. loebli (Fig. 103).

\section{Derivatio nominis}

The name refers to the conspicuously large eyes of this species. 


\section{Comparative notes}

From all other congeners with a distinct neck, this species is readily distinguished especially by the large eyes, the coarse puncturation of the posterior half of the abdominal terga III-V, and by the morphology of the aedeagus.

\section{Distribution and bionomics}

The fact that the types were collected in Taiwan and in Nepal (Map 8) suggests that $A$. megalops is very widespread in the Eastern Palaearctic region. The specimens were collected in April and May at altitudes of 1650 and $2050 \mathrm{~m}$.

\subsection{The formosana group}

The formosana group currently includes two species from Taiwan and Japan, which share the following synapomorphies: head and pronotum with distinct microsculpture, a short and dense pubescence of the forebody, abdominal terga III-VI in posterior half densely punctate. There are probably additional synaporphies in the morphology of the aedeagus, but the male of $A$. densepunctata is unknown. The formosana group is linked to the loebli group, presumably its adelphotaxon, by several derived characters: antennomere III shorter than II, ligula only apically bifid, mesosternal process broadly truncate, aedeagus with distinctly sclerotized internal structures (see also section 3.3).

\subsubsection{Amarochara formosana sp. n.}

Figs. 104-111, Map 8

\section{Type}

Holotype ơ: TAIWAN Ilan Hsien, Chyr Duan, 1100m, 19.IV.90, A. Smetana [T9] / Holotypus of Amarochara formosana sp. n. det. V. Assing 2000 (MHNG). Paratypes: 699 [1 우 without head]: same data as holotype (MHNG, cAss).

\section{Description}

Relatively large species, $3.7-4.1 \mathrm{~mm}$. Body blackish brown to blackish; posterior margins of abdominal terga and legs brown to dark brown; elytra dark yellowish brown. Head and pronotum with distinct, elytra with shallower microreticulation; posterior parts abdominal terga III-VI with very shallow and indistinct, terga VII and VIII with more distinct reticulation. Pubescence of forebody relatively short, but very dense and erect. Head of \pm subquadrate shape, slightly (approximately 1.10 times) wider than long, not dilated behind eyes; posteriorly without neck, posterior angles weakly marked; eyes large and convex, distinctly projecting from lateral outline of head, their diameter approximately as long as postgenae in dorsal view. Puncturation extremely fine, barely noticeable in the microreticulation. Genal carina present, but very fine. Antenna massive and long, antennomere I with pronounced and long dorso-apical furrow; antennomere III shorter 
than II, antennomere $\mathrm{V}$ moderately transverse (less so than in most congeners), connecting joints between antennomeres relatively narrow (Fig. 110). Third joint of maxillary palpi very long and slender (Fig. 109); ligula as in Fig. 108; anterior margin of labrum in the middle with two very short stout sensilla; mandibles very long and slender, right mandible with indistinct tooth and weakly serrate in dorsal molar region.

Pronotum $1.00-1.05$ times as wide as long and approximately 1.2 times as wide as head; maximal width approximately halfway between anterior angles and middle; lateral outline near posterior angles weakly concave or straight, posterior angles well-defined; puncturation extremely dense, more distinct than that of head.

Elytra at suture $0.80-0.85$ times the length of pronotum and approximately 1.4 times as wide as pronotum; posterior margin near posterior angles indistinctly sinuate; puncturation fine and dense. Legs long and slender; first metatarsomere as long as the combined length of the three following tarsomeres or nearly so. Mesosternum without median carina; mesosternal process apically broadly truncate.

Abdominal terga III-V with moderately deep and broad, tergum VI without anterior impression; sterna III-V anteriorly constricted; puncturation in anterior impressions of terga III-V and near anterior margin of tergum VI very dense and coarse; puncturation of terga III-V on remainder of tergal surface finer than in anterior impression, but rather coarse and dense (much more so than in $A$. loebh), interstices on average narrower than punctures; puncturation of terga VI and VII finer and sparser, but still very distinct. Tergum $X$ anteriorly not distinctly elongated, broadly truncate (Fig. 111); with very dense, stout, long and dark setae on all of its surface.

$\sigma^{*}$ : posterior margin of tergum VIII truncate, with a dense row of long thin marginal setae; posterior margin of sternum VIII convex with a very dense row of long and thin marginal setae and with interspersed long thin submarginal setae; median lobe of aedeagus with long and slender ventral process, at base of ventral process with pair of weakly prominent lateral folds; crista apicalis and crista proximalis present, but rather small; internal sac with very long tube and pair of weakly sclerotized apical structures of distinctive shape (Figs. 104-105); paramere unmodified, apical lobe of paramere broadly coniform and apically acute (Fig. 106).

q : tergum VIII of similar shape and chaetotaxy as in $\sigma^{*}$; posterior margin of sternum VIII weakly convex, row of marginal setae unmodified, thin, and short; spermatheca relatively small, with rather short, wide, and apically distinctly bent duct (Fig. 107).

\section{Derivatio nominis}

The name (Lat., adj.) is derived from Formosa, the former name of Taiwan.

\section{Comparative notes and comments}

A. formosana is distinguished from all its congeners, except for $A$. densepunctata (see the following species section), by a number of characters, especially the densely punctured pronotum, the distinct microsculpture of the whole forebody, the short, dense, and erect pubescence, the densely and coarsely punctured abdominal terga III-V, and by the primary sexual characters in both sexes. 


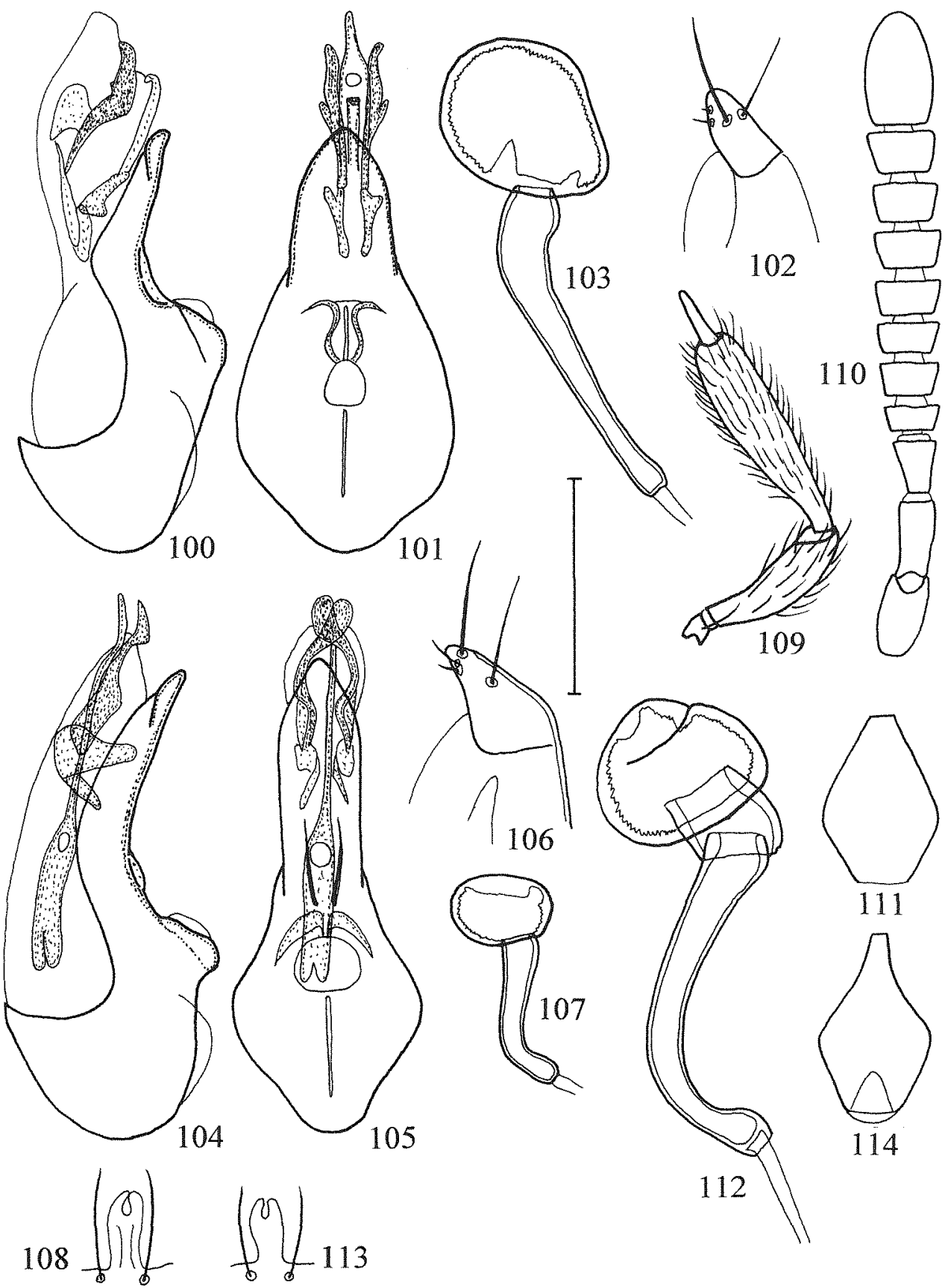

Figs. 100-114: Amarocbara megalops sp. n. (100-103), A. formosana sp. n. (104-111), and A. densepunctata sp. n. (112-114). Median lobe of aedeagus in lateral and in ventral view $(100,101,104,105)$; apical lobe of paramere $(102,106)$; spermatheca $(103,107,112)$; ligula $(108,113)$; maxillary palpus (109); antenna, pubescence omitted (110); tergum X, pubescence omitted (111, 114). Scale: 102, 106, 108, 112, 113; $0.1 \mathrm{~mm} ; 100,101$, 103-105, 107, 109: $0.2 \mathrm{~mm} ; 110,111,114: 0.4 \mathrm{~mm}$. 


\section{Distribution and bionomics}

The types were collected in Chihtuan (NE-Taiwan, Yilan Hsien) (Map 8) at an altitude of $1100 \mathrm{~m}$ in April.

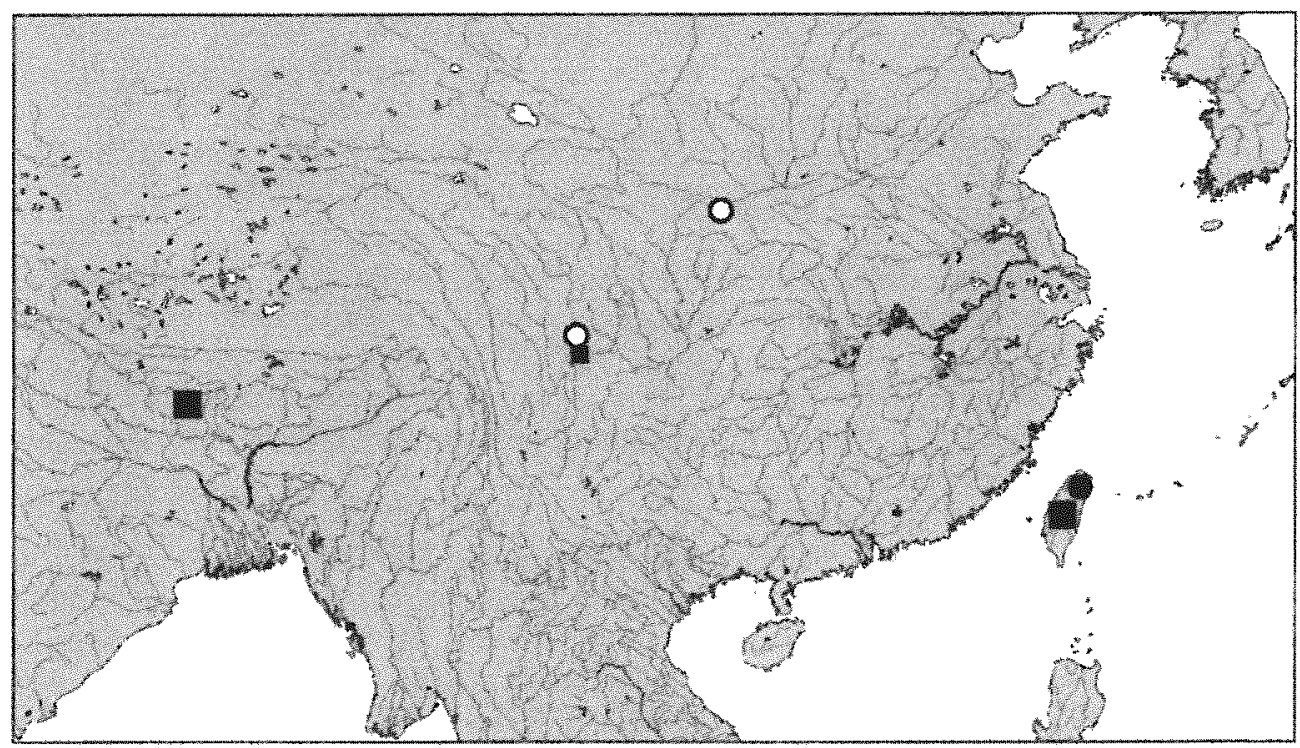

Map 8: Distribution of Amarachara megalops sp. n. (large squares), A. wrasei sp. n. (small square), A. armata sp. n. (open circles), and A. formosana sp. n. (filled circle).

\subsubsection{Amarochara densepunctata sp. n.}

Figs. 112-114

\section{Type}

Holotype ?: JAPON GUNMA, $4 \mathrm{~km}$ SW Tsumagoi, 1050 m, 18.7.1980, Löbl / Holotypus ot Amarochara densepunctata sp. n. det. V. Assing 2000 (MHNG).

\section{Description}

In size $(3.7 \mathrm{~mm})$, coloration, and general appearance somewhat resembling $A$. inermis. Head and antenna dark brown with the basal antennomere slightly lighter, pronotum, legs, and abdomen (except for the lighter tergal margins and abdominal apex) brown; elytra slightly lighter brown; maxillary palpi testaceous. Microreticulation on head weak, on pronotum very weak (visible only at high magnifications), on elytra absent, and on abdomen very shallow. Pubescence of forebody relatively short and dense, weakly erect, almost decumbent.

Head of subcircular shape, approximately as wide as long, not dilated behind eyes; posteriorly without neck, posterior angles weakly marked; eyes moderately large and weakly convex, weakly projecting from lateral outline of head, their diameter slightly shorter 
than postgenae in dorsal view. Puncturation very fine and rather sparse. Genal carina pronounced. Antenna massive and long, antennomere I with pronounced and long dorsoapical furrow; antennomere III slightly shorter than II, antennomeres IV-X of similar proportions as in $A$. formosana, but IV and V more transverse, and $\mathrm{X}$ less transverse and longer than IX, connecting joints between antennomeres relatively narrow. Maxillary palpi, labium, and labrum of similar morphology as in A. formosana (Fig. 113); mandibles apically less long and acute than in $A$. formosana; right mandible without tooth.

Pronotum 1.08 times as wide as long and approximately 1.4 times as wide as head; maximal width before the middle; lateral outline near posterior angles \pm straight, posterior angles rather well-defined; puncturation very fine, more ill-defined and much denser than that of head.

Elytra at suture 0.82 times the length of pronotum and approximately 1.2 times as wide as pronotum; postetior margin near posterior angles weakly, but noticeably sinuate; puncturation slightly granulose, very dense, and much more distinct than that of pronotum. Legs long and slender; first metatarsomere very long, longer than the combined length of the three following tarsomeres. Mesosternum without median carina; mesosternal process apically broadly truncate.

Anterior impressions of abdominal terga III-V of similar depth and breadth as in $A$. formosana, tergum VI without anterior impression; sterna III-V anteriorly constricted; puncturation in anterior impressions of terga III-V and near anterior margin of tergum VI very dense and coarse; puncturation on remainder of dorsal surface finer than in anterior impressions of terga III-V, but conspicuously coarse, somewhat granulose, and dense in comparison to other Amarochara species, interstices on terga III-VI distinctly narrower than punctures. Tergum $X$ anteriorly distinctly elongated, narrowly truncate; with very dense, stout, long and dark yellowish setae; posterior median area without setae (Fig. 114).

ơ: unknown.

$q$ : posterior margin of tergum VIII almost truncate and with rather dense row of marginal setae (broken off in the holotype); posterior margin of sternum VIII with dense row of short unmodified marginal setae; spermatheca of characteristic derived morphology, relatively small, with apically constricted capsule, the latter connected to duct by a short and broad tube; duct rather short, subapically thin and distinctly bent, and apically noticeably dilated (Fig. 112).

\section{Derivatio nominis}

The name (Lat., adj.) refers to the conspicuously dense puncturation of the abdomen.

\section{Comparative notes and comments}

A. densepunctata is distinguished from all its congeners especially by the dense puncturation of the abdomen and by the morphology of the spermatheca. It additionally differs from other Eastern Palaearctic species in the lighter coloration and the large pronotum, which is much wider than the head and only slightly narrower than the elytra.

\section{Distribution and bionomics}

The holotype was collected near Tsumagoi (Gunma, Honshu), some $140 \mathrm{~km}$ NW Tokyo at an altitude of approximately $1000 \mathrm{~m}$. 


\subsection{Amarochara splendens JARRIGE, 1952}

Figs. 122-126

Amarochara (Mniobates) splendens JARRIGE, 1952: $138 \mathrm{f}$.

\section{Type examined}

Holotype $\sigma^{*}$ [dissected and remounted]: Tamtierf, Gd Atlas (Kocher), $1400-4.48 /$ Amarochara splendens / TYPE / Muséum Paris Ex Collection J. Jarrige 1976 / Holotypus Amarochara splendens rev. V. Assing 2001 (MNHNP).

\section{Redescription}

Relatively large species, $4.6 \mathrm{~mm}$. Colour of body distinctive: head, pronotum, and abdomen blackish, except for the slightly lighter abdominal apex; elytra bicoloured, with the anterior and lateral margins blackish and with the posterior and interior two thirds of each elytron brightly rufous, thus forming a clear and large reddish spot; antennae and legs light brown to yellowish brown. Whole body without appreciable microsculpture. Pubescence of forebody relatively long, decumbent to suberect.

Head of subcircular shape, approximately as wide as long; eyes very large, moderately convex, weakly projecting from lateral outline of head, almost as long as postgenae in dorsal view; postgenae smoothly curved towards posterior margin. Puncturation moderately sparse and moderately fine, but distinct, with interspersed micropunctures. Genal carina present and distinct. Antennae very long and massive, similar to those in A. siculifera ASSING, antennomere I with dorso-apical furrow reduced, rery short and inconspicuous; antennomere III even slightly longer than II; IV and V moderately transverse; VI - X weakly transverse. Third joint of maxillary palpus very long and slender, similar to that in $A$. siculifera.

Pronotum large in relation to head, 1.15 times as wide as long and 1.37 times as wide as head; maximal with a short distance anterior to middle; posterior angles weakly defined; puncturation similar to that of head, but much denser, interstices mostly narrower than punctures.

Elytra relatively long, at suture 0.85 times the length of pronotum and approximately 1.20 times as wide as pronotum; posterior margin near posterior angles weakly sinuate; puncturation, especially in anterior half, coarse and very dense, interstices reduced to narrow ridges. Hind wings fully developed. Mesosternum without carina, mesosternal process long and apically acute. Legs very long and slender, metatarsus almost as long as metatibia and first metatarsomere as long as the combined length of the three following tarsomeres.

Abdominal terga III-V with deep anterior impressions, tergum VI without anterior impression; sterna III-V anteriorly weakly constricted; puncturation in impressions of terga III-V and near anterior margin of tergum VI very coarse and dense, on remainder of dorsal abdominal surface moderately dense and relatively coarse, denser and much coarser than in A. siculifera and A. forticornis. Tergum and sternum VIII long and slender. Tergum $\mathrm{X}$ with long anterior extension. 
$\sigma^{*}$ : posterior margin of tergum VIII moderately convex, that of sternum VIII strongly convex, with a dense row of relatively long marginal and a sparse row of very long submarginal thin setae (Figs. 125-126); median lobe of aedeagus long and slender, at base of ventral process with distinct median process best seen in lateral view; crista apicalis completely, crista proximalis partly reduced; internal tube with relatively long basal part; internal sac without distinctly sclerotized structures (Figs. 122-123); apical lobe of paramere slightly modified, the two apical setae very short (Fig. 124).

q: unknown.

\section{Comparative notes and comments}

A. splendens is distinguished from other species of the genus by the distinctive coloration alone. Based on the morphology of the median lobe of the aedeagus (especially the presence of a median process at the base of the ventral process), the morphology of the antennae and mouthparts, the extremely long and slender legs, and other morphological
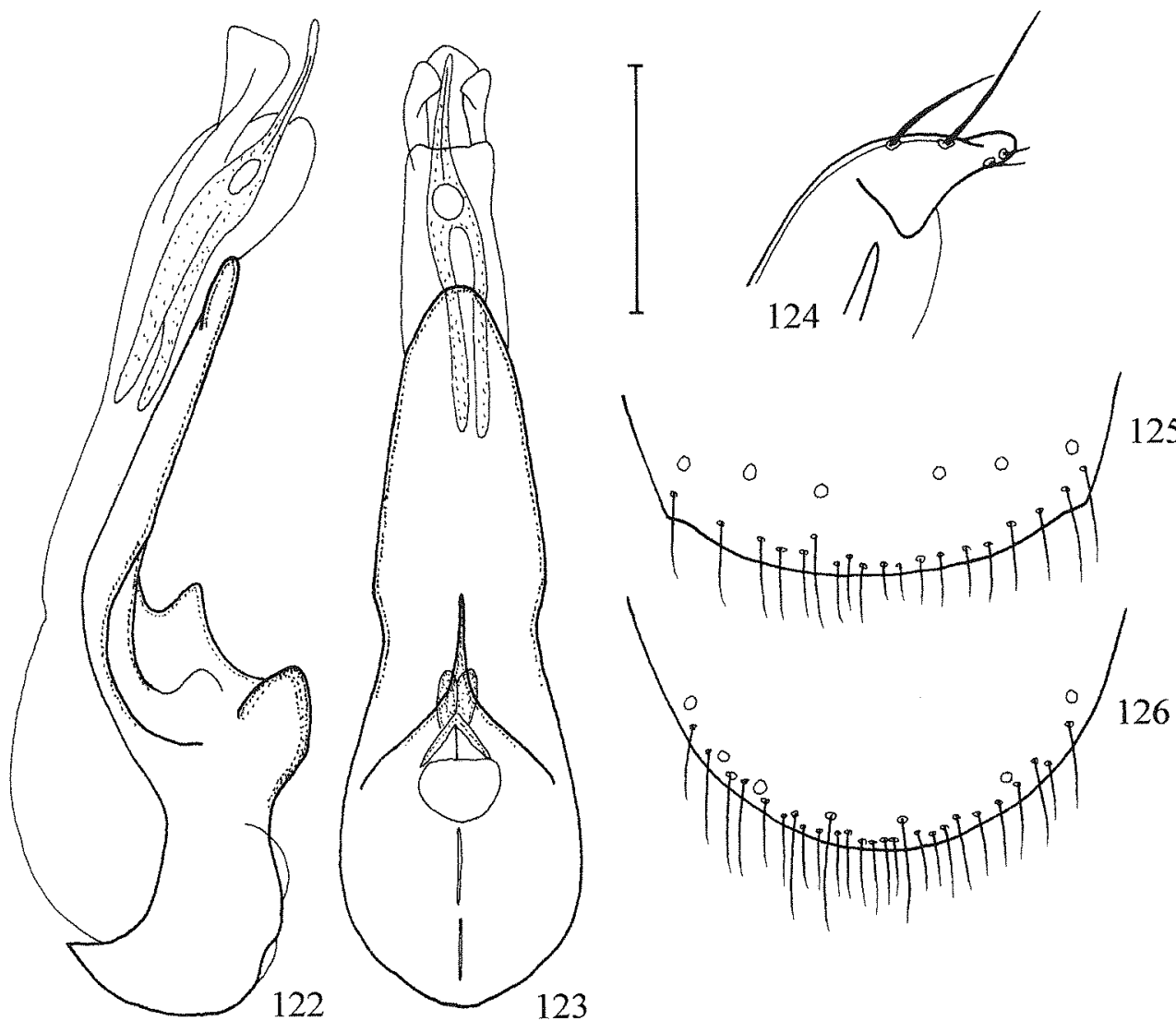

Figs. 122-126: Amarachara splendens J ARRIGE. Median lobe of aedeagus in lateral and in ventral view (122, 123); apical lobe of paramere (124); posterior margin of $\sigma^{*}$ tergum VIII (125); posterior margin of $\delta$ sternum VIII (126); long setae omitted in 125 and 126. Scale: 124:0.1 mm; 122-123, 125-126:0.2 mm. 
characters, $A$. splendens is closely related to $A$. siculifera sp.n. and $A$. inermis sp.n. from the eastern Mediterranean. It is additionally separated from these species by much larger eyes, the more distinct puncturation of head, pronotum, elytra, and of the posterior areas of the abdominal terga III - VII, by the more slender median lobe of the aedeagus, and by the different shape and chaetotaxy of the apical lobe of the paramere.

\section{Distribution and bionomics}

The species is known only from the type locality in the Haut Atlas, Marocco, where it was collected at an altitude of $1400 \mathrm{~m}$ in April.

\subsection{Amarochara tingitana JARRIGE, 1952}

Amarochara (Mniobates) tingitana JARRIGE, 1952: 138.

\section{Comments}

The holotype of this species was apparently lost in the process of posting. The labels read as follows: 94 Tanger / Maroc ex Musae E. Vaucher 1908 / Amarochara tingitana Jarr. / TYPE / Muséum Paris Ex Collection J. Jarrige 1976 (MNHNP). Unfortunately, the original description is based on a single specimen, so that an interpretation of this species and a neotype designation will have to wait until additional material becomes available.

5. Key to the Amarochara species of the Holarctic region

The following key does not account for $A$. tingitana JARRIGE (see chapter 4.7).

1. Head posteriorly without a distinct neck. ........................................................ 2

Head posteriorly with a distinct neck. Eastern Palaearctic region.................... 19

2. Relatively large species, $3.0-5.0 \mathrm{~mm}$. Abdominal tergum VI with distinct anterior impression (though this impression is shallower than those of terga III-V). Elytral hind margins near posterior angles strongly sinuate. Mesosternum with median carina. Aedeagus and spermatheca as in Figs. 71-72, 74. W-Palaearctic (Map 6).

A. forticormis (LACORDAIRE)

Abdominal tergum VI at most with very indistinct anterior impression. Elytral hind margins near posterior angles not or only weakly sinuate. Mesosternum usually without, only in one species from Spain with carina. Genitalia different. 3

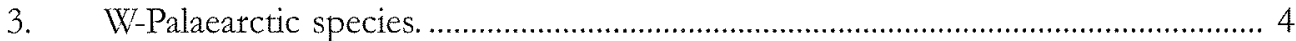

E-Palaearctic or Nearctic species. ....................................................................... 12 
4. Smaller species, $2.2-3.2 \mathrm{~mm}$. Genal carina completely absent. Antennae relatively slender (Fig. 5). Eyes very large and bulging, distinctly projecting from lateral outline of head. Head of \pm distinctly subquadrate shape. Aedeagus and spermatheca as in Figs. 8-10. Palaearctic region from Western Europe to Siberia and China (Maps 1-2).

A. umbrosa (ERICHSON)

Larger species. Genal carina present, though often very fine. Antenna more massive. Eyes smaller and/or less bulging, usually distinctly shorter than postgenae in dorsal view and weakly projecting over lateral outline of head; the latter without or with weakly marked posterior angles. Genitalia different. W-Palaearctic species with more restricted areas of distribution. 5

5. Large species, length approximately $4.5 \mathrm{~mm}$. Coloration distinctive: elytra bicoloured, with the anterior and lateral margins blackish and with the posterior and interior two thirds of each elytron rufous, thus forming a large reddish spot; head, pronotum, and abdomen blackish. Whole body without microsculpture. Elytra with dense and coarse puncturation. $0^{7}$ : median lobe of aedeagus very long and slender and, at base of ventral process, with distinct median process (Figs. 122-123). Morocco.

A. splendens JARRIGE Coloration different, elytra not bicoloured. Head and pronotum either of lighter colour (except for dark specimens of $A$. cribripennis) or head with distinct mictosculpture. 0 : median lobe of aedeagus without median process at base of ventral process and/or less slender. 6

6. Large species, $3.8-4.6 \mathrm{~mm}$. Pronotum large in relation to head, $1.15-1.25$ times as wide as long and approximately 1.4 times as wide as head or even wider. Antennomere III as long as II. Puncturation of anterior impressions of abdominal terga III-V very coarse and dense. Eastern Mediterranean.

Smaller species. Pronotum less than 1.4 times as wide as head, only in one species from NW-Africa of similar relative width. Antennomere III often shorter than II. Puncturation of anterior impressions of abdominal terga III-V less coarse and less dense. Absent from the Eastern Mediterranean, except for one species with fine puncturation in anterior impressions of terga III-V. 8

7. Median lobe of aedeagus in lateral view with distinct median process at base of ventral process (Figs. 58-59). Central southern Anatolia to Lebanon (Map 5).....

A. siculifera sp. n.

Median lobe of aedeagus of with longer ventral process and in lateral view without median process at base of ventral process (Figs. 62-63). Istael (Map 5)......

A. inermis sp. n.

8. Coloration of body uniformly blackish. Head and pronotum with distinct mictosculpture. Puncturation in impressions of terga III-V and near anterior margin of tergum VI coarse and dense. Spermatheca as in Fig. 68. Tunisia.

A. caeca sp. n. 
Colour of body at least partly lighter. Head and pronotum with distinct microsculpture only in one light-coloured species.

9. Antennae more massive and longer (at least approximately $1.2 \mathrm{~mm}$ ) (Fig. 69). Metatarsomere I at least slightly longer than the combined length of the three following tarsomeres (Fig. 70). Puncturation in the anterior impressions of terga III-V coarse and dense. Western Mediterranean. 10

Antennae less massive and shorter than $1.2 \mathrm{~mm}$. Metatarsomere I shorter than the combined length of the three following tarsomeres.

10. Mesosternum with median carina. Antennae with much more transverse antennomeres; antennomere $\mathrm{V}$ approximately 3 times as wide as long; eyes much shorter than postgenae in dorsal view; pronotum smaller and less broad, approximately 1.3 times as wide as head; elytra shorter, at suture approximately 0.75 times the length of pronotum. Legs much shorter; approximate length of metatibia and metatarsus $0.62 \mathrm{~mm}$ and $0.51 \mathrm{~mm}$. Spermatheca as in Fig. 78. Northern Spain. .

A. carinata sp. n.

Mesosternum without median carina. Antennae with less transverse antennomeres (Fig. 69); antennomere $\mathrm{V}$ approximately 2 times as wide as long; eyes almost as long as postgenae in dorsal view; pronotum large and broad, approximately 1.4 times as wide as head; elytra shorter, at suture approximately 0.85 times the length of pronotum. Legs much longer; approximate length of metatibia and metatarsus $0.74 \mathrm{~mm}$ and $0.63 \mathrm{~mm}$, respectively (Fig. 70). Sexual characters unknown. Morocco.

A. crassicornis (QUEDENFELDT)

11. Head and pronotum with shallow, but distinct microsculpture. Colour of body yellowish brown to light brown. Antennae shorter and less massive (Fig. 50). Abdomen (especially the anterior terga) with sparser and finer puncturation. Aedeagus as in Figs, 44-45. Widespread from Anatolia in the southeast to France and Great Britain in the west and northwest (Map 3).

A. bonnairei (FAUVEL)

Head and pronotum without distinct microsculpture. Body colour usually darker. Antennae longer and more massive (Fig. 57). Abdomen (especially the anterior terga) with denser and coarser puncturation. Aedeagus as in Figs. 52-53. Corsica and Sardinia (Map 4).

A. cribripennis MULSANT \& REY

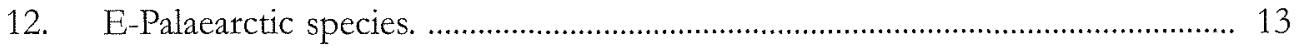

Nearctic species

13. Small species with finely carinate genal carinae. Abdomen with anterior impressions of terga III-V shallow and posteriorly ill-defined; sterna III-V with weak anterior constriction; puncturation distinctive: on terga III-VI weakly granulose; on terga III-V rather dense and well-defined, but not very coarse, not coarser and denser in anterior impressions than on remainder of tergal surface; on tergum VI slightly coarser and denser than on terga III-V, and on tergum VII finer and sparser 
than on terga III-VI. Pubescence of forebody short, dense, and decumbent. Pronotum almost as wide as elytra. Antenna slender (similar to that in $A$. umbrosa). Spermatheca as in Fig. 28. Northern India.

A. sororcula CAMERON

Anterior impressions of abdominal terga III-V deeper and with coarser puncturation than posterior tergal area. Pubescence of forebody longer, less dense, and usually \pm suberect. Antenna - except for the widespread $A$. umbrosa - more massive.

14. Small species with completely absent genal carinae and slender antennae (Fig. 5). Head of subquadrate shape and with \pm distinctly marked posterior angles. Aedeagus and spermatheca as in Figs. 8-10. Widespread Palaearctic species; in the Eastern Palaearctic region known from Siberia and China (Maps 1-2).

\section{A. umbrosa (ERICHSON)}

Mostly larger species. Genal carina sometimes teduced, but rudiments usually visible at least near occiput. Antennae more massive. Head often with weakly marked or indistinct posterior angles. Genitalia different.

15. Large and light-coloured species. Genal carina pronounced. Pronotum very large, approximately 1.4 times as wide as head. Puncturation in anterior impressions of terga III-V and near anterior margin of tergum VI very dense and coarse; puncturation of posterior parts of tergal surface finer than in anterior impressions of terga III-V, but conspicuously coarse, somewhat granulose, and dense. Spermatheca of characteristic morphology (Fig. 112). Japan.

A. densepunctata sp. n.

Colour of body usually darker. Genal carina fine or \pm reduced. Pronotum $<1.3$ times as wide as head. Puncturation of posterior areas of abdominal terga III-V less coarse, less dense, and not granulose. Absent from Japan.

16. Smaller species, $<3.5 \mathrm{~mm}$. Genal carina reduced, rudiments usually visible only near occiput and/or below eyes. Pronotum with moderately dense puncturation and with some shine. Microsculpture of head and pronotum shallow. Aedeagus as in Figs. 21-22; spermatheca as in Fig. 24. Northern India, Nepal.

A. beterogaster CAMERON

Larger species, 3.7-4.1 $\mathrm{mm}$. Pronotum with conspicuously dense fine puncturation and with dense, short, and suberect pubescence; almost mat. Microsculpture on head and pronotum distinct. Aedeagus and spermatheca as in Figs. 104-105, 107). Taiwan (Map 8).

A. formosana sp. n.

17. Colour of body \pm uniformly brown or ferrugineous, only abdominal tergum VI and sometimes head and antennae \pm infuscate. Antennae shorter, less than 0.75 $\mathrm{mm}$. Eyes shorter and less bulging, postgenae at least 1.5 times as long as eyes in dorsal view. Puncturation of abdomen very fine, only in anterior impressions of terga III-V somewhat more distinct. Spermatheca as in Fig. 38. Iowa.

A. inquilina (CASEY) 
At least head and abdomen distinctly darker. Antennae longer than $0.75 \mathrm{~mm}$. Eyes larger and more bulging, distinctly projecting from lateral outline of head; postgenae less than 1.5 times as long as eyes in dorsal view. Puncturation of abdomen very distinct.

18. Larger species, $3.0-3.8 \mathrm{~mm}$. Coloration on the whole usually darker, pronotum at most only slightly lighter than the dark brown to blackish head; antennae not distinctly bicoloured. Antennae longer and with less transverse antennomeres; antennomeres VII-IX approximately twice as wide as long (Fig. 37). Pronotum relatively large, 1.25-1.30 times as wide as head. Elytra relatively short, at suture approximately 0.75 times as long as pronotum. Legs distinctly longer, length of metatarsus even in small specimens at least $0.37 \mathrm{~mm}$; first metatarsomere very elongate, as long as the combined length of the three following tarsomeres or nearly so (Fig. 35). Abdomen with more clearly defined and less dense puncturation, and with pronounced shine. $\sigma^{*}$ : aedeagus of completely different shape, with much longer and more slender ventral process (Figs. 29-30). $\%:$ spermatheca smaller (Fig. 32). Indiana, Kansas, Georgia.

A. fenyesi BLATCHLEY Smaller species, $2.8-3.2 \mathrm{~mm}$. Pronotum light brown to brown, distinctly lighter than the blackish head; antennae distinctly bicoloured, dark brown to blackish, with yellowish to ferrugineous basal antennomeres. Antennae shorter and with. more transverse antennomeres; antennomeres VII-IX almost 3 times as wide as long. Pronotum relatively smaller. Elytra longer, at suture $>0.80$ times as long as pronotum. Legs much shorter, length of metatarsus approximately $0.30 \mathrm{~mm}$; first metatarsomere of similar length as in $A$. umbrosa, shorter than the three following tarsomeres. Abdomen with less defined and much denser puncturation, and therefore with reduced shine. $\sigma^{*}$ : median lobe of aedeagus much shorter and broader ventral process (Figs. 39-40). o : spermatheca larger (Fig. 42). Kansas.

\section{A. brevios sp. n.}

19. Abdominal terga III and IV with one, tergum $V$ with two conspicuous transverse series of dense and coarse punctures in posterior half. Small species. Nepal (Map 7). A. seriepunctata $\mathrm{sp} . \mathrm{n}$.

Abdominal terga III-V without distinct transverse series of dense and coarse puncture in posterior half. Larger species.

20. Aedeagus and paramera strongly modified. Ventral process of median lobe short and small in relation to basal part, in lateral view with short process or obtuse projection near base of ventral process; internal sac with several strongly sclerotized structures. Apical lobe of paramere distinctly flattened (Figs. 84, 85, 95)...

Aedeagus and paramere not strongly modified. Ventral process of median lobe longer in relation to basal part, in lateral view without short process or obtuse projection near base of ventral process; internal sac with weakly sclerotized structures. Apical lobe of paramere not distinctly flattened. 
21. Ventral process of median lobe longer, in lateral view near base of ventral lobe with short and acute process; sclerotized structures of aedeagus of distinctive shape (Figs. 82-83, 86); apical lobe of paramere not transverse, apically rounded (Figs. 84-85); spermatheca as in Fig. 87. Nepal (Map 7).

A. Loebli PACE

- Ventral process of median lobe shorter, in lateral view near base of ventral lobe with obtuse projection; sclerotized structures of aedeagus different (Figs. 92-93); apical lobe of paramere transverse, apically truncate (Figs. 94-95). China (Sichuan, Shaanxi) (Map 8).

A. armata sp. n.

22. Antennae much longer, antennomeres VII-X approximately 1.5 times as wide as long. Eyes larger, approximately as long as postgenae in dorsal view. Posterior halves of abdominal terga III- $V$ with distinct, though not very dense puncturation. Aedeagus and spermatheca as in Figs. 100-101, 103). Nepal and Taiwan (Map 8)..

A. megalops sp. $\mathrm{n}$.

Antennae distinctly shorter, antennomeres VII-X $>2$ times as wide as long. Eyes smaller, somewhat shorter than postgenae in dorsal view. Posterior halves of abdominal terga III-V with very fine and sparse puncturation. Aedeagus and spermatheca as in Figs. 96-97, 99). China (Sichuan) (Map 8).

A. wrasei sp. n.

6. Species excluded from Amarochara

\subsection{Dasygnypeta velata (ERICHSON, 1837)}

Calodera flavipes MOTSCHULSKY, 1858: 259.

\section{Types examined}

Lectotype $q$ : Paris / Calodera flavipes Motsch. / LECTOTYPUS Calodera flavipes Motsch., V. E Semenov dsg. 1998 / Dasyngnypeta relata (Er.) V. B. Semenor det. 1998 (ZMMU).

Paralectotype $\%:$ St. Germain, am Vaud[]]-ufer / Calodera flavipes Motch. Gallia / PARALECTOTYPUS Calodera flavipes Motsch., V. E Semenov dsg. 1998 / Dasyngnypeta velata (Er.) V. B. Semenov det. 1998 (ZMMU).

V. B. Semenov's identification is here confirmed. Calodera flavipes MOTSCHULSKY is consequently a junior synonym of Dasygnypeta velata (ERICHSON, 1837) and not of Amarochara forticornis (LACORDATRE) as indicated by BERNHAUER \& SCHEERPELTZ (1926). 


\subsection{Alevonota japonica (CAMERoN, 1933), comb. n.}

Amarochara (s. str.) japonica CAMERON, 1933: 218.

\section{Type examined}

Holotype $o$ [hind legs missing]: Kobe Mayasan 26.IX.23 Lewis / Amarochara japonica Cam. TYPE / M. Cameron. Bequest. B.M. 1955-147. / Holotype / Amarochara japonica Cam. P.M. Hammond det. 1973 HOLOTYPE / Alevonota japonica (Cameron) det. V. Assing $2000(\mathrm{BMNH})$.

\section{Additional material examined}

$10^{7}, 2$ 우, Japan, Nara pref., Nara, 27.-31.VII.1980, leg. Besuchet (MHNG, cAss).

\section{Comments}

The holotype of A. japonica is not a member of the oxypodine genus Amarochara, but belongs to the Athetini and is here attributed to the genus Alevonota THOMSON based on its general appearance (slender body, slender pronotum), the rather stout antennae, the mouthparts (deeply bifid ligula), and the morphology of the primary sexual characters, especially of the spermatheca. It differs from Western Palaearctic congeners particularly in the shape of antennomere I, which is rather large, somewhat flattened, and which has a pronounced wide dorsal furrow.

\section{3. "Amarochara" flavicornis BERNHAUER, 1907}

Amarocbara flavicomis BERNHAUER, 1907: $412 \mathrm{f}$.

\section{Type examined}

Holotype o [dissected prior to present study]: Japan. Sauter. Yamanaka. 23.3.1905 / Amarochara flavicornis Bh. Typus / Chicago NHMus M.Bernhauer Collection (FMNH).

\section{Comments}

Prior to the present study, the mouthparts and the genitalia of the holotype had been dissected and glued to the mounting label. Various solvents were tried, but a glue had been used that proved to be resistant to all these agents, so that it was impossible to assess many important characters properly and to examine them under a compound microscope (mouthparts, primary and secondary sexual characters, pro- and mesotarsi, ventral aspect of body). The holotype is definitely no Amarochara; its antennae, abdomen, metatarsi, maxillary palpi, and aedeagus are of completely different morphology. Judging from the aedeagus and other characters, it apparently belongs to an athetine genus, characterized by relatively massive antennae (with weakly transverse antennomeres IV-X, short and stout antennomeres II and III, a short antennomere XI, and a distinct dorsal furrow on antennomere I), maxillary palpi with the third joints distinctly dilated, relatively short and stout mandibles, a transverse pronotum, a short first metatarsomere (not longer than second), and anteriorly weakly impressed abdominal terga 
III-V. The generic affiliations of this species may remain uncertain until new material becomes available for examination.

\subsection{Calodera caseyi nom. n., comb. n.}

Nasirema bumilis CASEY, 1893: $308 \mathrm{f}$.

Amarochara (Lasiochara) bumilis: BERNHAUUR \& SCHEERPELTZ (1926).

\section{Type examined}

Holotype $q$ [two legs missing, remounted]: Penn / 5-5-5 / Nasirema humilis / CASEY bequest 1925 / TYPE USNM 39747 / Holotype Nasirema humilis Casey rev. V. Assing $2000 /$ Calodera caseyi nom. n. det. V. Assing 2000 (USNM).

\section{Comments}

Nasirema bumilis, the type species of Nasirema CASEY, 1893, was previously attributed to Amarochara (e. g. BERNHAUER \& SCHEERPELTZ 1926). However, an examination of the holotype revealed that the species belongs to Calodera MANNERHEIM, 1830 and not to Amarochara. Consequently, Nasirema, which has previously been regarded as a syno-nym of Amarochara, is here placed in the synonymy of the senior name Calodera. $C$. bumilis CASEY is a secondary junior homonym of C. bumilis ERICHSON, 1837 (which again is a junior synonym of Calodera protensa MANNERHEIM, 1830), so that the replacement name Calodera caseyi nom. $\mathrm{n}$. is proposed for C. bumilis (CASEY). C. caseyi belongs to the group of species without posteriorly distinctly constricted head. From C. lapponica SAHLBERG and C. protensa MANNERHEIM, the species is distinguished by its distinctly smaller size (in this respect similar to C. aethiops (GRAVENHORST)), the lighter coloration (light brown, with the elytra and the appendages testaceous), the shorter antennae with more transverse antennomeres V-X (antennomere more than twice as wide as long), and by the denser and coarser puncturation of the pronotum.

\subsection{Calodera parviceps (CASEY, 1893), comb. $n$.}

Nasirema pamiceps CASEY, 1893: 309.

Amarochara parviceps; as synonym of $A$. (s. str.) umbrosa: BERNHAUER \& SCHEERPELTZ (1926).

\section{Type examined}

Syntype $q$ [remounted]: R. I / Nasirema parviceps / CASEY bequest 1925 / TYPE USNM 39748 / Syntype Nasirema parviceps Casey rev. V. Assing 2000 / Calodera cf. rufescens Kraatz det. V. Assing 2000 (USNM).

\section{Additional material examined}

1 ㅇ [slightly teneral]: locality illegible, ex coll. Casey and identified as Nasirema bumilis by Casey (USNM). 


\section{Comments}

The original description of the species is based on an unspecified number of types. The single female syntype in the Casey collection is not designated as lectotype, because Calodera species can often only be safely identified based on the male genitalia and because there is a chance that male syntypes may be found in the future. The syntype and the specimen listed as additional material very much resemble the European $C$. rufescens KRAATZ. Whether or not they are in fact conspecific, however, can only be decided when males of $C$. parviceps become available.

\subsection{Pseudocalea korbi (BerNHAUER, 1902), comb. n.}

Figs. 115-120

Amarochara korbi BERNHAUER, 1902: $253 \mathrm{f}$.

\section{Type examined}

Holotype o : Kleinasien Korb. / Korbi Bernh. Type / Chicago NHMus. M. Bernhauer Collection / Holotypus Amarochara korbi Bernhauer rev. V. Assing 2000 / Pseudocalea korbi Bernhauer det. V. Assing 2000 (FMNH).

A. korbi is here attributed to the genus Pseudocalea LUZE of the Aleocharini. Previously, two species of Pseudocalea were known: P. brevicornis (KRAATZ) and P. angulata (EPPELSHEIM) (ASSING, 2000; ASSING \& WUNDERLE, 1997). With these species, P. korbi shares a similar morphology of the mouthparts (Figs. 118-119), various external characters such as the slender body, the ventral aspect of the thorax (mesosternum anteriorly with a distinct $V$-shaped emargination), the similar shape and chaetotaxy of the of sternum VIII (Fig. 120), and the general morphology of the aedeagus (Figs. 115-116). It is, however, readily distinguished from both species by much smaller body size (approximately $3.8 \mathrm{~mm}$, general appearance similar to Amarochara forticornis, but with a much smaller and differently shaped pronotum), much lighter colour (head, pronotum, antennae, margins of abdominal terga, and abdominal apex ferrugineous, head and remainder of abdomen slightly darker), shorter antennae with a transverse antennomere IV, a dis-tinctly granulose puncturation of the elytra, the deeper emargination of the elytral hind margin near external angles, the much shorter tarsi, the shape and internal structures of the median lobe of the aedeagus (Figs. 115-116), and the more slender apical lobe of the paramere (Fig. 117). For illustrations of the mouthparts and sexual characters of $P$. brevicornis and $P$. angulata see ASSING (2000) and ASSING \& WUNDERLE (1997, 1998). 

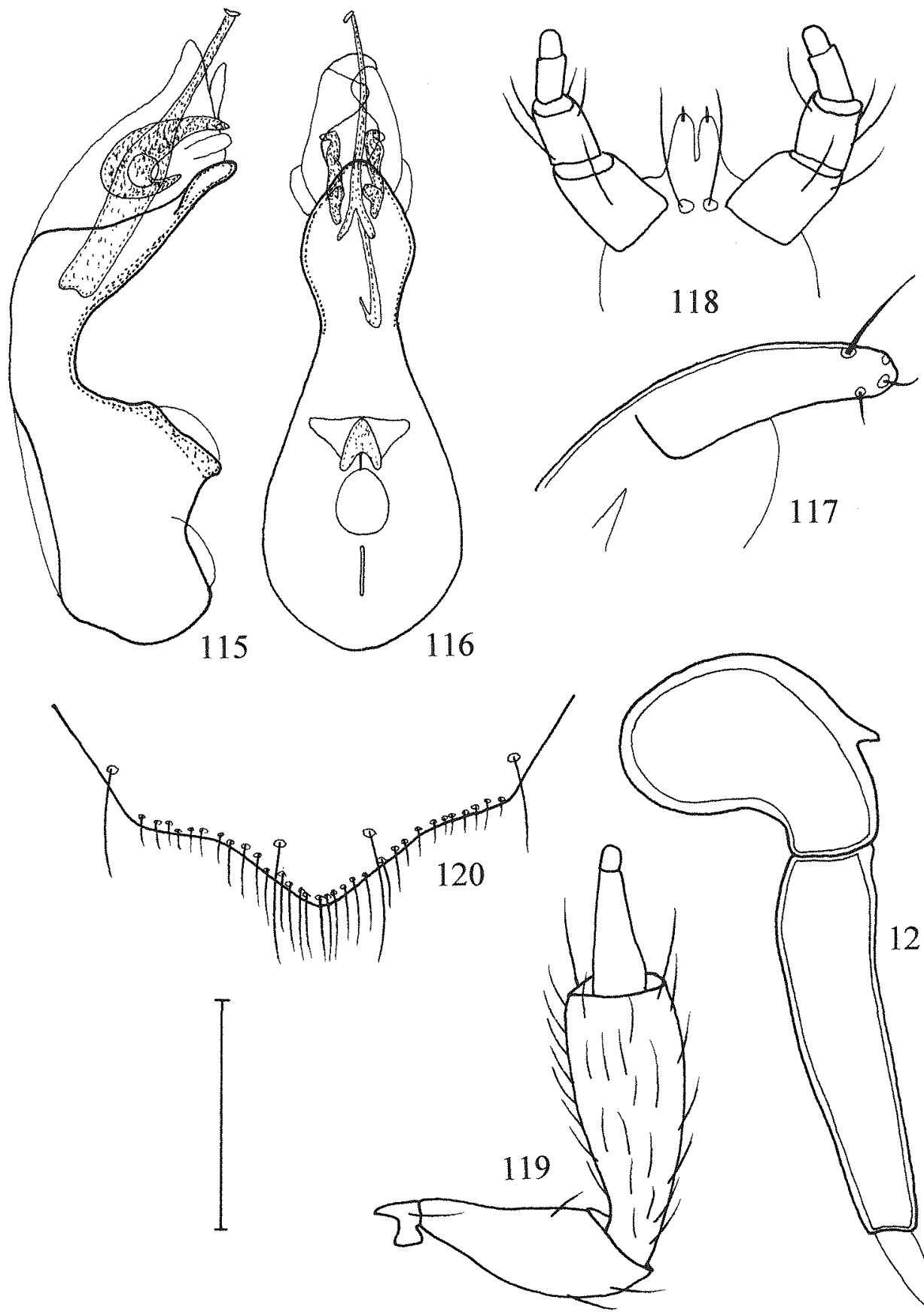

Figs. 115-121: Pseudocalea korbi (BERNHAUER) (115-120) and Aleochara ocaleoides (BERNHAUER) (121). Median lobe of aedeagus in lateral and in ventral view $(115,116)$; apical lobe of paramere (117); labium (118); maxillary palpus (119); posterior margin of $\sigma^{*}$ sternum VIII (120); spermatheca (121). Scale: 117-119, 121: $0.1 \mathrm{~mm} ; 115,116,120: 0.2 \mathrm{~mm}$. 


\subsection{Aleochara (Ceranota) ocaleoides (BERNHAUER, 1902), comb. n.}

Fig. 121

Amarocbara ocaleoides BERNHAUER, 1902: 252.

\section{Type examined}

Holotype o: Sassisecte [P], Asia min. Korb / 60 / c. Epplsh. Steind. d. / ocaleoides Bernh. Type / TYPUS / Holotypus Amarochara ocaleoides Bernhauer rev. V. Assing 2000 / Aleochara ocaleoides (Bernhauer) det. V. Assing (NHMW).

\section{Comments}

An examination of the holotype revealed that $A$. ocaleoides belongs to the genus Aleochara GRAVENHORST, 1802, subgenus Ceranota STEPHENS, 1839. The mesonotum anteriorly has a short rudiment of a median carina; for a description of other external characters see BERNHAUER (1902). The spermatheca is illustrated in Fig. 121.

\subsection{Aleochara (Ceranota) subtumida (HochнUтH, 1849)}

Calodera brunnea MOTSCHULSKY, 1860: 582 f.

Ocalea reitteri BERNHAUER, 1900: 46 f., syn. $n$.

Ocalea (Sorecocepbala) reitteri: BERNHAUER (1902).

\section{Types examined}

Calodera brunnea: Lectotype $q$ : brunnea / Achalzik [almost illegible] Abes-Tum. [according to original description "Akhaltzik" "Abes-Touman"] / 341 / Calodera brunnea Motsch. Armenia / LECTOTYPUS Calodera brunnea Motsch., V. E Semenov dsg. 1998 / Aleochara subtumida (Hochh.) V. B. Semenov det. 1998 (ZMMU).

Ocalea reitteri: Lectotype $\%$, here designated: Kaukas Leder / Reitteri Brh von Reitter Type / Chicago NHMus M. Bernhauer Collection / Messung Nr. 15 / Lectotypus of Ocalea reitteri Bernhauer desig. V. Assing 2000 / Aleochara subtumida (Hochhuth) det. V. Assing 2000 (FMNH). Paralectotype \&: Kaukas Leder / coll. Reitter / Paratypus 1900 Ocalea Reitteri Bernhauer (HNHM).

\section{Comments}

V. B. Semenov's identification of the lectotype of Calodera brunnea is here confirmed. The name is a junior synonym of Aleocbara (Ceranota) subtumida (HOCHHUTH, 1849) and not of Amarochara forticornis (LACORDAIRE) as indicated by BERNHAUER \& SCHEERPELTZ (1926).

The description of Ocalea reitteri is based on two syntypes, which were found in the Bernhauer collection at the FMNH and in the Reitter collection at the HNHM. The specimens are conspecific with Aleochara subtumida, so that the following synonymy is established: Aleocbara subtumida $($ HOCHHUTH, 1849) $=$ Ocalea reitteri BERNHAUER, 1900, syn. n. 
In order to fix a single name-bearing type and to preserve the present interpretation of the species, the syntype from the Bernhauer collection is designated as lectotype. Illustrations of the genitalia of $A$. subtumida are provided by GUSAROV (1991), who studied the types.

Two years after the description of O. reitteri, BERNHAUER (1902) attributed the species to the new subgenus Sorecocepbala, with $O$. reitteri as the sole representative and type species by monotypy. No further species of Sorecocephala have become known. Since $O$. reitteri is a synonytn of a species of Aleochara, the following synonymy is here established:

Aleochara GravenHORST, 1802 = Sorecocephala BERnHAUER, 1902, syn. n.

\section{Acknowledgements}

I am indebted to all the colleagues indicated in section 2 and, in addition, to Dr. R. Outerelo, Madrid, for the loan of material. In particular, I would like to thank Andreas Pütz (Eisenhüttenstadt), Michael Schülke, and David Wrase (Berlin) for the generous gift of some holo- and paratypes. Frank Köhler contributed some ecological observations and additional records. Benedikt Feldmann and Michael Schülke kindly proofread the manuscript. Dr. Alexey Solodornikor, St. Petersburg, kindly assisted in making a loan of the types deposited at the MNHNP possible after all. Finally, I would like to thank the editorial staff of the journal for their apparently endless patience and their offer to incorporate all those additions that occurred after the (first) submission of the manuscript.

\section{References}

ÁDAN, L. 1987: Staphylinidae of the Kiskunság National Park (Coleoptera). - In: MAHUNKA, S. (ed.): The fauna of the Kiskunság National Park II, Akadémia Kiadó Budapest: 126-128.

AssiNG, V. 1988: Die Kurzflügelkäferfauna (Coleoptera: Staphylinidac) ausgewählter Grün-, Ruderal- und Kleingartenflächen im Stadtgebiet Hannovers: Ein Beitrag zur Faunistik und Öloologie einer Großstadt. Ber. naturhist. Ges. Hannover 130: 111-131.

ASsING, V. 1994a: Zur Kurzflügelkäferfauna xerothermer Flächen im südlichen Niedersachsen (Coleoptera: Staphylinidae), - Göttinger naturkundl. Schriften 3: 7-31.

AsstNG, V. 1994b: Pseudonicrodota jelineki und weitere faunistisch bemerkenswerte Aleocharinen von der Porta Westfalica im Weserbergland (Staphyl.) (Kl. Mitt. Nt. 2136). - Entomol. Blätter 90: 123-124.

AssING, V. 1996: A revision of the European species of Calodera MANNERHEIM (Coleoptera, Staphylinidae, Aleocharinae), - Beitr. Ent. Berlin 46: 3-24.

Assing, V. 1998: A revision of the Palaearctic species of Zoosetha MULSANT \& REY and Poromniusa G.ANGLBAUER (Coleoptera, Staphylinidae, Aleocharinae). - Dtsch. entomol. Z. 45: 73-94.

AssING, V. 1999: A revision of Ilyobates KRAATZ, 1856 (Coleoptera: Staphylinidae, Aleochatinae, Oxypodini). - Beitr. Ent. Berlin 49: 295-342.

Assing, V. 2000: A revision of the species of Euryalea MULSANr \& RFY and Pseudocalea LuZE (Coleoptera, Staphylinidae, Aleocharinae). Supplement I. - Entomol. Blätter 96: 3-8.

Assing, V. 2001: A revision of Callicerus GravenHorst, 1802, Psendosemiris MACHulKA, 1935, and Saphocallus SHARP, 1888 (Coleoptera: Staphylinidae, Aleocharinae, Athetini). - Beitr. Ent. Keltern 51: 247-334.

Assing, V. \& WunderLe, P. 1997: A revision of the species of Euryalea Mulsant \& REY, Psendoutlea LuzE and Oyota SHARp (Coleoptera, Staphylinidae, Aleocharinae). - Entomol. Blätter 93: 93-126.

Assing, V. \& WUNDERLE, P. 1998: A revision of the species of Euryalea MUISANT \& REY, Pseudocalea LuZE and Ogota SHARP (Coleoptera, Staphylinidae, Aleocharinae): Figures 2-13, - Entomol. Blätter 94, Suppl.: 1-24. 
BENICK, L. 1920: Beiträge zur Kenntnis der Tierwelt norddeutscher Quellgebiete. II. Coleoptera. - Arch. Naturgesch. 85 (1919): 299-316.

Bernhauer, M. 1900: Neue Staphyliniden (Coleopt.) aus dem Kaukasus und den angrenzenden Ländern. Wien. Ent. Zeit. 19: 46-55.

BERNHAUER, M. 1902: Die Staphyliniden der paläarktischen Fauna. I. Ttibus: Aleocharini. (Il. Theil.). - Verh. zool.-bot. Ges. Wien 52: 87-284.

BERNHAUER, M. 1907: Zur Staphylinidenfauna von Japan. - Veth. zool.-bot. Ges. Wien 57: 371-414.

BERNHAUER, M. \& SCHEERPELTZ, O. 1926: Staphylinidae VI. - In: JUNK, W. \& S. SCHENKLING (eds.): Coleopterorum Catalogus, pars 82, Berlin: 499-988.

BLACKWELDER, R. E. 1952: The genetic names of the beetle family Staphylinidae with an essay on genotypy. - Smiths. Inst. U.S. Nat. Mus. Bull. 200: 1-483.

BLATCHLEY, W. S. 1910: On the Aleocharinae known to occur in Indiana. - In: The Coleoptera of Indiana, Bull. Indiana Dep. Geol. Nat. Res. 1: 337-367.

BÖrME, J. 1996: Käfer in der Bodenstreu mitteleuropäischer Laubwälder. - Entomol. Blätter 92: 37-63.

BOHÁC, J. \& Kol. 1993: Staphylinidae. - In: JELÍNEK, J. (ed.): Check-list of Czechoslovak Insects IV (Coleoptera), Folia Heyrovskyana, Suppl. 1: 39-62.

CAMERon, M. 1933: New species of Staphylinidae (Col.) from Japan, - Entomogist's Monthly Mag. 69: 208219.

CAMERon, M. 1939: The Fauna of British India, including Ceylon and Burma. Coleoptera. Staphylinidae. Vol. IV. Parts I-II. - London: 691 pp.

CAsey, T. L. 1893: III. - Coleopterological Notices. V. - Annals N. Y. Acad. Sci. 7: 281-607.

CASEY, T. L. 1906: Observations on the staphylinid groups Aleocharinae and Xantholinini, chiefly of America. - Trans. Acad. Sci. St. Louis 16 (2): 125-434.

CoIfFAIT, H, 1973: Staphylinides nouveaux ou mal connus du Maroc. - Bull. Soc. Sci. Nat. Phys. Maroc 53: 269-292.

COIFFAIT, H. 1976: Staphylinidae récoltés par M. Cerruti dans la région méditerranéenne orientale (Coleoptera). - Fragm. Entomol. 12: 81-101.

ERrCHSON, G. F. 1837: Die Käfer der Mark Brandenburg. 1. Bd., 1. Abt., Berlin, 384 pp.

ERICHSON, G. F. 1839: Genera et species staphylinorum, insectorum coleopterorum familiae. Berlin: 1-400.

FAUVEL, A. 1865: Énumération des insectes recueillis en Savoie et en Dauphiné (1861-1863) et descriptions d'espèces nourelles. - Bull. Soc. Linn. Norm. 9: 253-321.

FENYES, A. 1920: Coleoptera: Fam. Staphylinidae, subfam. Aleocharinae. Genera Insectorum, fasc. 173b: 111-414, Bruxelles.

FOWLER, C. 1888: The Coleoptera of the British Islands. Vol. II. Staphylinidae. - London: 444 pp.

FrANZ, H. 1938: Aus der Praxis des Käfersammlers. XXXIII. Eine Käferfauna aus Fuchsbauen des nördlichen Burgenlandes, - Koleopt. Rdsch. 24: 30-32.

FRANZ, H. 1970: Die Nordost-Alpen im Spiegel ihrer Landtierwelt. Eine Gebietsmonographie. Band III. Coleoptera 1. Teil, umfassend die Familien Cicindelidae bis Staphylinidae. - Innsbruck-München: 501 pp.

GAEDIKE, R. 1995: Collectiones entomologicae (1961-1994). - Nova Suppl. Ent. Berlin 6: 3 83.

GaMArRA, P. 1987: Citas nuevas para España de estafilinoideos. Familia Aleocharidae. Subfamilia Oxypodinae (Coleoptera: Polyphaga). - Anal. Biol. 11: 67-70.

GANGlbaUer, L. 1895: Die Käfer von Mitteleuropa. 2. Band: Staphylinidae und Pselaphidae. - Wien: 850 pp.

GEISER, R. 1998: Rote Liste der Käfer (Coleoptera). - In: Bundesamt für Naturschutz (ed.): Rote Liste gefährdeter Tiere Deutschlands, Bonn-Bad Godesberg: 168-230.

GÜRLICH, S.; SUIKAT, R. \& ZIEGLER, W. 1995: Katalog der Käfer Schleswig-Holsteins und des Niederelbegebietes. - Verh. Ver. Naturw. Heimatforsch. Hamburg 41: 1-111.

Gusarov, V. 1991: New and little-known Palaearctic Staphylinidae (Coleoptera, Staphylinidae). Note 2 [title translated from Russian]. - Vestnik Leningrad Univ, Ser. 3 Biol., fasc. 3: 3-12.

GUSAROV, V. 1992: New and little-known Palaearctic Staphylinidae (Coleoptera, Staphylinidae) [title translated from Russian]. - Vestnik St. Petersburg Univ., Ser. 3 Biol. 1 (3): 1-25. 
Haberman, H. 1983: Beitrag zu "Enumeratio Coleopterorum Fennoscandiae et Daniae"(1979) über die Staphyliniden Estlands. - Notul. Entomol. 63: 97-110.

HANSEN, M. 1996: Katalog over Danmarks biller. - Entomol. Medd. 64 (1/2): 1-231.

Hansen, M.; Lijejehut, H.; Mahler, V. \& Pedersen, J. 1995: 14. tillæg til "Fortegnelse over Danmarks biller" (Coleoptera), - Ent. Meddr. 63: 21-50.

HANSEN, V. 1964: Fortegnelse over Danmarks biller (Coleoptera). - Entomol. Medd. 33: 1-507.

Horion, A. 1951: Verzeichnis der Käfer Mitteleuropas (Deutschland, Österreich, Tschechoslovakei) mit kurzen faunistischen Angaben. 1. Abteilung: Caraboidea, Palpicornia, Staphylinoidea, Malacodermata, Sternoxia, Fossipedes, Macrodactyla, Brachymera. - Alfred Kernen Verlag, Stuttgart, I-VII: 1-266.

HorION, A. 1967: Faunistik der mitteleuropäischen Käfer. Bd. XI: Staphylinidae, 3. Teil: Habrocerinae bis Aleocharinae (ohne Subtribus Athetae). - Überlingen-Bodensee: 419 pp.

Hugentobler, H. 1966: Beitrag zur Kenntnis der Käfer der Nordostschweiz. - Naturwiss. Ges. St. Gallen: $248 \mathrm{pp}$.

ICZN 1999: International Code of Zoological Nomenclature. Fourth Edition. London: XXIX + 306 pp.

JARRIGE, J. 1952: Brachélytres nouveaux ou mal connus de la faune circaméditerranéenne. - Ann. Soc. ent. France 119: 117-139.

Joy, N. H. 1932: A practical handbook of British beetles. Vol. I. - London: 622 pp.

KACHE, P. 1998: Die Besiedlung urbaner Lebenstäume dutch Kurzflügelkäfer (Coleoptera, Staphylinidae) unter besonderer Berücksichtigung des Dispersionsvermögens und des Reproduktionserfolgs der Arten. - Dissertation Universität Osnabrück: 180 pp.

KoCH, K. 1968: Käferfauna der Rheinprovinz. Decheniana (Bonn), Beih. 13: 1-382.

KOCH, K. 1974: Erster Nachtrag zur Käferfauna der Rheinprovinz. - Decheniana (Bonn) 126: 191-265.

KocH, K. 1989: Die Käfer Mitteleuropas. Ökologie, Band 1. - Krefeld: 440 pp.

KOCIAN, M. 1993: The staphylinid beetles of Prague (Coleoptera: Staphylinidae). - Klapalekiana 29: 91-98.

KOHLER, F. 1997: Eine neue Schwemmtechnil für faunistisch-ölologische Untersuchungen der Käferfauna an Gewässerufern. - Entomol. Blätter 92 (1996): 137-161.

KÖHER, F. 2000: Totholzkäfer in Naturwaldzellen des nördlichen Rheinlandes. Vergleichende Studien zur Totholzkäferfauna Deutschlands und deutschen Naturwaldforschung. Naturwaldzellen in NordrheinWestfalen VIII. - Schr. LÖBF/LAfAO NRW (Recklinghausen) 18: 1-354.

KÖHLER, F. \& KLAUSNITZER, B. 1998: Verzeichnis der Käfer Deutschlands. - Entomol. Nachr. Ber. (Dresden) Beih. 4: 1-185.

KÖHLER, F. \& StunPF, T. 1992: Die Käfer der Wahner Heide in der Niederrheinischen Bucht bei Köln (Insecta: Coleoptera). Fauna und Artengemeinschaften, Veränderungen und Schutzmaßnahmen. Decheniana Beih. (Bonn) 31: 499-593.

KRAATZ, G. 1856: Naturgeschichte der Insekten Deutschlands. Bd. 2. Staphylinii. - Berlin, 1-376.

LACordaire, J. T. 1835: In: BoIsduval, J. B. A. D. DE \& LACORDAIRE, J. T.: Faune entomologique des environs de Paris ou species général des insectes que se trouvent dans un tayon de quinze a vingt lieues aux alentours de Paris. I, - Paris: 696 pp.

LATTIN, G. DE 1967: Grundriss der Zoogeographie. - Gustax Fischer Verlag, Stuttgart: 602 pp.

LIKOVSKX́, Z. 1985: Kratsie príspevky a informácie. Drabcici (Coleoptera, Staphylinidae) v záznamech exkurzí Professora Jana Roubala z let 1939-1969. - Zbor. Slov. nár. Múz., Prír. Vedy 31: 171-187.

LINKE, M. 1907: Verzeichnis der in der Umgebung von Leipzig beobachteten Staphyliniden. - Sitzungsber. Natuforsch. Ges. Leipzig 1906-1907: 1-54.

LINKE, M. 1927: Zweiter Beitrag zur Kenntnis der Staphyliniden des Freistaates Sachsen und einiger Grenzgebiete. - Coleopterol. Centralbl. 1: 355-364.

LINKE, M. 1962: Dritter Beitrag zur Kenntnis der Staphyliniden Sachsens und des nordwestlichen Grenzgebietes. - Entomol. Nachr. 6: 89-99, 105-107.

LOHSE, G. A. 1967: Die Aleocharini (s. lat.) des Niederelbegebietes und Schleswig-Holsteins (Col. Staphylinidae). - Verh. Ver. Naturx: Heimatforsch. Hamburg 36: 39-50.

LoHse, G. A. 1974: Tribus 15-19 (Schistogenini-Aleocharini). - In: Freude, H.; HARDE, K.W. \& LOHSE, G. A.: Die Käfer Mitteleuropas, Bd. 5, Krefeld: 221-292. 
LUCHT, W. H. 1987: Die Käfer Mitteleuropas. Katalog: - Krefeld: 342 pp.

LUIGIONI, P. 1929: I coleotteri d'Italia. Catalogo sinonimico-topografico-bibliografico. - Memorie Accad. pontificia Sci., (II) 13: 1-1160.

LUNDBERG, S. 1995: Catalogus Coleopterorum Sueciae. - Naturhistoriska Riksmuseet, Entomologiska Föreningen: without pagination.

MAJZIaA, O. \& JASZAY, T. 1997: Taxocenózy drobčikor (Coleoptera, Staphylinidae) v pôde lu•ných lesov rieky Moravy. - Fol. faun. Slov. 2: 61-69.

MOTSCHULSKY, V. 1858: Enumération des nouvelles espèces de coléoptères rapportés de ses voyages. - Bull. Soc. Nat. Moscou 31: 204-264.

MOTSCHULSKY, V. 1860: Enumération des nouvelles espèces de coléoptères rapportés de ses voyages. - Bull. Soc. Nat. Moscou 33: 539-588.

MulsanT, M. E. \& REY, C. 1875: Tribu des brévipennes: Famille des Aléochariens - suite - Sixième branche. - Aléocharaires (suite). - Ann. Soc. Linn. Lyon, ser. 2, 21: 1-403.

Normand, H. 1934: Contribution au Catalogue des Coléoptères de Tunisie (4. fascicule). - Bull. Soc. Hist. nat. Afr. Notd 25: 356-390.

NovAK, P. 1952: Kornjaši Jadranskog Ptimorja (Coleoptera). - Jugoslavenska Akademija Znanosti I Umjetnosti: $521 \mathrm{pp}$.

OSELLA, G. \& ZANETTI, A. 1975: La coleotterofauna dei nidi di Talpa europaea L. nell'Italia settentrionale a nord del fiume Po. - Boll. Zool. Agr. Bachicoltura, Ser. II, 12 (1974): 43-200.

OUTERELO, R. 1980: Los Staphylinoidea de la Sierra de Cazorla. - In: VIEDMA, M. G. DE: Fauna de Cazorla. Invertebrados. - Minist. Agtic. Inst. Nac. Conserv. Nat. Monografias 23: 53-71.

PACE, R. 1984: Aleocharinae dell Himalya [sic] LI. Contributo alla conoscenza delle Aleocharinae (Coleoptera, Staphylinidae). - Annls. Soc, ent. Fr. (N. S.) 20: 309-339.

PACE, R. 1992: Aleocharinae nepalesi del Museo di Ginevra Parte VII (conclusione): Oxypodini e Aleocharini (Coleoptera, Staphylinidae) $\left(115^{\circ}\right.$ Contributo alla conscenza delle Aleocharinae), - Rerue suisse Zool. 99 : 263-342.

PACE, R. 1998: Aleocharinae della Cina: Parte I (Coleoptera, Staphylinidae). - Rerue suisse Zool. 105: 139 220.

PALM, T. 1972: Skalbaggar. Coleoptera, Kortringar: Fam. Staphylinidae, Underfam. Aleocharinae (Alewonota - Timotus), Häfte 7, pp. 301-467. - In: Entomologiska Föreningen I Stockholm (ed.): Svensk Insektfauna 9, Stockholm.

Peez, A. v. \& Kahlen, M. 1977: Die Käfer von Südtirol. Faunistisches Verzeichnis der aus der Protrinz Bozen bisher bekanntgewordenen Koleopteren. - Beilageband 2 zu den Veröffentlichungen des Museum Ferdinandeum, Innsbruck: $525 \mathrm{pp}$.

PORTA, A. 1926: Fauna Coleopterorum Italica, 2. Staphylinoidea. - Piacenza: 1-405.

QUEDENFELDT, M. 1882: Diagnosen neuer Staphylinen aus dem Mittelmeer-Faunengebiet. - Berliner Ent. Zeitschr. 26: 181-183.

Rambousek, F. G. 1910: O bulharských Staphylinidech. - Sitzungsber. königl. böhm. Ges. Wiss., Mathem.naturw. Kl. 1909 (4): 1-23.

RYE, E. C. 1865: Description of a species of Oxypoda new to science. - Entomologist's Monthly Mag. 1: 212. SAINTE-Clatre DEtiLLe, J. 1914: Catalogue critique des coléoptères de la Corse. - Caen: 561 pp.

SCHEERPELTZ, O. 1959: VIII.Teil Staphylinidae (Col.). - In: BEIER, M.: Zoologische Studien in West-Griechenland. - Sitzungsber. mathem.-naturw. Kl., Abt. I. 167 (1958): 363-432.

SCHEERPEITZ, O. 1967: Wissenschaftliche Ergebnisse der zoologischen Studienfahrt von Dr. A. SmetanaPraha nach Albanien im Jahre 1958 (Coleoptera, Staphylinidae-Aleocharinae). - Acta Entomol. Mus. Nat. Pragae 37: 517-550.

SCHeERPELTZ, O. 1968: Catalogus Faunae Austriae. Teil XV fa: Coleoptera-Staphylinidae. - Östert. Akad. Wiss., Wien: $279 \mathrm{pp}$.

SCHMcAus, M. 1969: Zur Koleopterenfauna des Hunsrücks. 6. Beitrag. - Entomol. Blätter 65: 154-159.

SCHOLZE, P. \& JUNG, M. 1994: Beiträge zur Faunistik der Kurzflügler (Col., Staphylinidae) im Nordharz und Vorland II. Unterfamilie Aleocharinae. - Entomol. Nachr. Ber. 38: 7-12. 
SCHOLZE, P.; UHLIG, M. \& VOGEL, J. 1999: Kommentierte Artenliste der Kurzflügler der Brockenregion (Coleoptera: Staphylinidae). - Abh. Ber. Naturk. Magdeburg 22: 89-97.

SCHÜLKE, M.; UHLIG, M. \& ZERCHE, L. 1992: Kurzflügler (Staphylinidae). - In: Gefährdete Tiere im Land Brandenburg - Rote Liste, Ministerium für Umwelt, Naturschutz und Raumordnung des Landes Brandenburg, Potsdam: 155-174.

SEEvERS, C. H. 1978: A generic and tribal tevision of the North American Aleocharinae (Colcoptera: Staphylinidae). - Fieldiana (Zoology) 71: 1-289.

SIEDE, D. 1992: Die Käferfauna des NSG Koppelstein. - Mitt. Arb.gem. Rhein. Koleopterologen (Bonn) 2: 3-40.

SilfyerberG, H. 1992; Enumeratio Coleopterorum Fennoscandiae, Daniae et Baltiae. - Helsingin Hyönteisvaihtoyhdistys, Helsingfors Entomologiska Bytesförening, Helsinki: 94 pp.

STRZEL.CZYK, P. 1988: Staphylinidae (Col.) aus großstädtischen Habitaten I. - Entomol. Nachr. Ber. 32: 227231.

THOMSON, C. G. 1858: Försök till uppställning af Sveriges Staphyliner - Öfv. Kön. Vet-Akad. Förh. 15: 27 40.

VÁSÁRHELYT, T. 1985: Békés megye bogárfaunája III. Staphylinidae 2. - Hydrophilidae (Coleoptera). - Folia Entomol. Hung. 46: 247-277.

VOGEI, J. 1982: Faunistisch bedeutsame und für die DDR neue Staphylinidae (Coleoptera) aus der Oberlausitz, Teil II. - Abh. Ber. Naturkundemus. Görlitz 55, 6: 1-26.

WÖRNDLE, A. 1950: Die Käfer von Nordtirol. - Schlern-Schriften, Bd. 54, Innsbruck: 388 pp.

ZANETTI, A. 1995: Habrocerinae, Trichophyinae, Tachyporinae e Aleocharinae (generi 148-314), pp. 33-58. In: Ciceroni, A., PUthZ, V. \& ZANETTI, A.: Coleoptera, Polyphaga III (Staphylinidae), Checklist delle specie della fauna italiana, Calderini Bologna, Fasc. 48: 1-65.

ZERCHE, L. 1980: Faunistisch interessante Staphylinidae aus der DDR (Coleoptera). - Entomol. Nachr. 24: $145-165$.

ZIEGLER,W; SUIKAT, R. \& GÜRICH, S. 1994: Rote Liste der in Schleswig-Holstein gefährdeten Käferarten. - Landesamt für Naturschutz und Landschaftspflege, Kiel: 96 pp.

\author{
Author's address: \\ VOLKER ASSING \\ Gabelsbergerstr. 2 \\ D-30163 Hannover \\ Germany \\ E-mail: vassing.hann@t-online.de
}

